\title{
EVALUACIÓN ECONÓMICA DEL TRATAMIENTO QUIRÚRGICO DE LA OBESIDAD
}

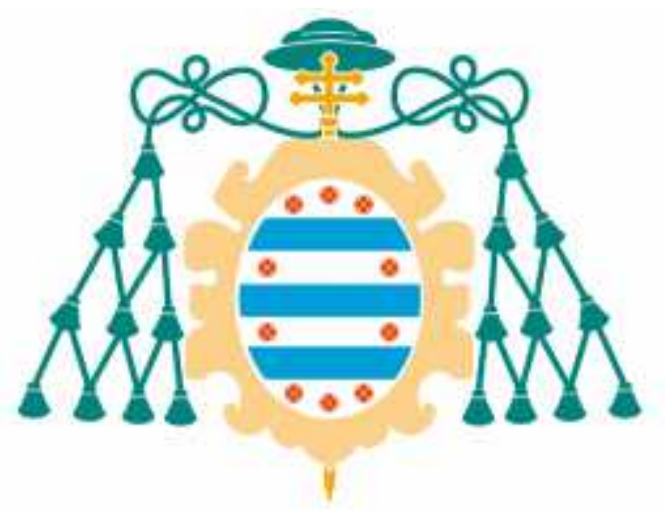

\section{Universidad de Oviedo}

PROGRAMA DE DOCTORADO:

Investigación en Cirugía y Especialidades Médico-Quirúrgicas 



\section{EVALUACIÓN ECONÓMICA DEL TRATAMIENTO QUIRÚRGICO DE LA OBESIDAD}

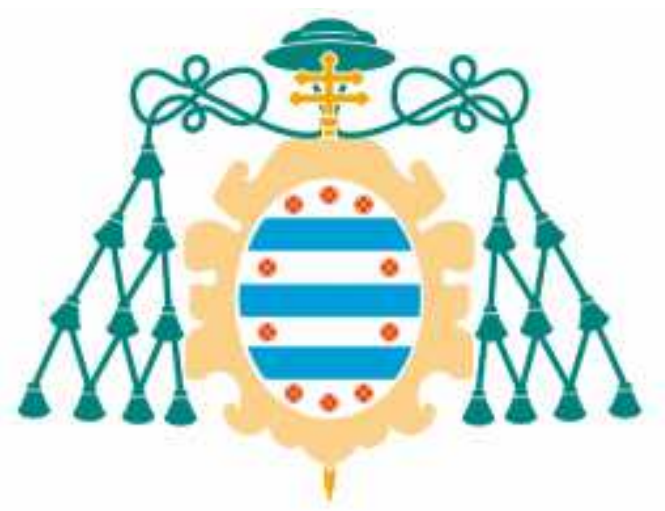

\section{Universidad de Oviedo}

PROGRAMA DE DOCTORADO:

Investigación en Cirugía y Especialidades Médico-Quirúrgicas 




\section{RESUMEN DEL CONTENIDO DE TESIS DOCTORAL}

\begin{tabular}{|l|l|}
\hline 1.- Título de la Tesis & \\
\hline Español: & Inglés: \\
EVALUACIÓN ECONÓMICA DEL TRATAMIENTO & ECONOMIC EVALUATION OF \\
QUIRÚRGICO DE LA OBESIDAD & SURGICAL TREATMENT OF OBESITY \\
\hline
\end{tabular}

\begin{tabular}{|c|c|}
\hline 2.- Autor & \\
\hline $\begin{array}{l}\text { Nombre: } \\
\text { JOSE LUIS RODICIO MIRAVALLES }\end{array}$ & DNI: \\
\hline $\begin{array}{l}\text { Programa de Doctorado: } \\
\text { INVESTIGACIÓN EN CIRUGÍA Y ES }\end{array}$ & DES MÉDICO-QUIRÚRGICAS \\
\hline $\begin{array}{l}\text { Órgano responsable: } \\
\text { CIRUGÍA Y ESPECIALIDADES MÉDI }\end{array}$ & GGICAS \\
\hline
\end{tabular}

RESUMEN (en español)

\section{INTRODUCCIÓN:}

La cirugía bariátrica es el único tratamiento efectivo de la obesidad mórbida demostrado a largo plazo, lo que da un poco de luz a una patología que de otra manera está llamada a agravarse.

Para conocer el coste del procedimiento quirúrgico disponemos de distintos métodos de evaluación. Con el ahorro generado por la mejoría de las comorbilidades tras la intervención, nos puede justificar el empleo de más recursos para el tratamiento quirúrgico de la obesidad.

\section{OBJETIVOS:}

Evaluación económica y resultados a corto plazo de un programa de cirugía bariátrica.

Estimación de gastos generados en el ingreso para la cirugía, mediante dos modelos diferentes; el tradicional, con los códigos GRD (Grupos Relacionados por el Diagnóstico) modelo top-down y el de imputación de coste por paciente y episodio (bottom-up), y comparación de los mismos.

Identificar los pacientes que más se benefician de este tratamiento.

\section{PACIENTES Y METODOLOGÍA:}

Estudio observacional, descriptivo, longitudinal y retrospectivo, de los pacientes intervenidos de cirugía bariátrica y a los que se les realizó un bypass gástrico, en el Hospital Universitario Central de Asturias entre los años 2003 y 2012. El seguimiento se establece en dos años.

Calculamos de manera individualizada el coste para cada uno de los pacientes intervenidos, así como hacemos uso del coste según el código de GRD. 
media de 43,5 años, peso medio en primera consulta de $131,8 \mathrm{~kg}$, y un índice de masa corporal medio (IMC) de $49,9 \mathrm{~kg} / \mathrm{m}^{2}$. El $92,2 \%$ presentó algún tipo de comorbilidad relacionada con la obesidad: $53,4 \%$ hipertensión arterial (HTA), $27,7 \%$ diabetes mellitus (DM), 23,7\% dislipemia (DL), 58\% síndrome de apnea e hipopnea del sueño (SAHS), 34,9\% artrosis y 40\% depresión.

El coste medio del ingreso calculado por GRD fue de $6.545,9 €$ y el calculado por paciente de $10.572,2 €$. El BPG se realizó por abordaje laparoscópico en $118(38,4 \%)$, que en 9/118 (7,6\%) de los casos precisó conversión a cirugía abierta. El tiempo medio de utilización del área quirúrgica fue de 287,7 minutos. La estancia media fue de 11,2 días.

El resultado medio a los dos años en porcentaje de sobrepeso perdido (PSP) fue de $69,3 \%$. Por comorbilidades la resolución de las mismas a los dos años fue: HTA 117/155 (75,5\%), DM 73/82 (89\%), DL 61/69 (88,4\%), SAHS 149/166 $(89,8 \%)$, artrosis $22 / 68(32,4 \%)$ y depresión $46 / 103(44,7 \%)$. De los pacientes medicados pasamos a los dos años de 2,86 a 0,78 fármacos por paciente. En el cuestionario de calidad de vida BAROS 224/273 (82\%) de los pacientes tuvieron un resultado muy bueno o excelente.

Los pacientes que más se beneficiaron de esta cirugía en el aspecto clínico fueron los más jóvenes y de menor IMC. Pacientes con DM tuvieron peor pérdida de peso, pero junto a los que tenían HTA y SAHS presentaron más resolución de comorbilidades. En el aspecto económico los más caros fueron los de mayor IMC, los de más estancia, los que tuvieron un balón intragástrico (BIG) previo y los que presentaron complicación.

Realizamos un cálculo estimativo del ahorro que pudiera suponer en nuestro entorno sanitario la disminución de fármacos tras dos años de la cirugía, que representó $2.025 €$ por paciente intervenido, y $4.433 €$ por paciente intervenido si padecía todas las comorbilidades analizadas.

\section{CONCLUSIONES:}

La cirugía bariátrica resuelve patologías graves y costosas, hasta la fecha irreversibles con otros tratamientos. A los dos años, en pacientes con pluripatologías el ahorro sólo en fármacos podría compensar los gastos inherentes al tratamiento quirúrgico.

El perfil de paciente que más se beneficia de este tipo de cirugía es el paciente joven, con menor IMC. Los pacientes con DM, SAHS e HTA responden mejor, mientras que aquellos con artrosis y depresión responden peor.

El coste por proceso mediante GRD se muestra insuficiente a la hora de hacer una correcta evaluación económica, recomendamos un método de estimación de coste por paciente. 


\section{RESUMEN (en Inglés)}

\section{INTRODUCTION:}

Obesity surgery is the only effective treatment for extreme obesity, with demonstrated long-term positive outcomes, shedding some light for a pathology that seems otherwise bound to worsen in the near future.

In this study, we present different assessment methods to estimate the cost of inpatient surgical procedure and the potential cost-savings generated with comorbidities improvement after surgery, which can justify the allocation of more resources in the surgical treatment of obesity.

\section{OBJECTIVES:}

Economic evaluation and short-term results in a bariatric surgery program.

Estimation of costs per surgery with two different methods; the traditional, with DRG (Diagnostic Related Group) codes (top-down) and the imputation of costs per patient and episode (bottom-up) and comparison between the two.

Identification of patients who benefit most from this treatment.

\section{METHODS:}

This is an observational, descriptive, longitudinal and retrospective study. Eligible patients undergo bariatric surgery (gastric bypass) in the Hospital Universitario Central of Asturias, between 2003 and 2012. Patients included in the study are followed-up for two years.

We compute individualized cost per patient and use DRG codes, using as reference rates and costs from 2013.

\section{RESULTS:}

Our study included 307 patients. Of these, $77.8 \%$ were women, average age was 43.5 years old, average baseline weight $131.8 \mathrm{~kg}$ and average BMI 49.9 $\mathrm{kg} / \mathrm{m} 2$. About $92 \%$ of patients had some kind of comorbidities associated with obesity: $53.4 \%$ high blood pressure (HBP), $27.7 \%$ diabetes mellitus (DM), $23.7 \%$ dislipemia (DL), 58\% sleep apnea-hypopnea syndrome (SAHS), 34.9\% arthrosis and $40 \%$ depression.

The average cost per hospitalization computed with DRG codes was $6545.9 €$ and the average cost per patient was $10572.2 €$. Gastric bypass was performed by laparoscopic approach in 118 (38.4\%) patients, with conversion to open surgery in 9 cases $(7.6 \%)$. The mean operating room time was 287.7 minutes and the mean length of stay (LOS) was 11.2 days.

Two years after the procedure, the overall percentage of excess weight loss (\%EWL) was $69.3 \%$ and comorbidities resolved in many cases: HBP $117 / 155$ (75.5\%), DM 73/82 (89\%), DL 61/69 (88.4\%), SAHS 149/166 (89.8\%), arthrosis 
$22 / 68(32.4 \%)$ and depression 46/103 (44.7\%). The number of medications also decreased during this period, from 2.86 to 0.78 per medically treated patient. Finally, the quality of life survey, evaluated by BAROS, showed a very good or excellent result for $224 / 273(82 \%)$ patients.

The surgery had the best clinical results among younger patients and in terms of effectiveness, those with lower pre-operative BMI seemed to do better. Diabetic patients had the worst weight loss but together with HBP and SAHS patients, experienced the most comorbidities resolution, whereas arthrosis and depression were associated with worse evolution after treatment. In economic terms, the most expensive cases were those with higher pre-operative BMI, longer LOS, previous intragastric balloon and with complications post-surgery. We also estimated the cost reduction associated with the observed decrease on the number of medications after two years: $2025 €$ per patient undergoing surgery and $4433 €$ per patient with all pre-operative comorbidities considered in the study (HBP, DM, DL, SAHS and depression).

\section{CONCLUSIONS:}

Bariatric surgery resolves serious and costly pathologies that were otherwise irreversible with other type of treatments until recently. At two years, patients with multiple pathologies saving drugs could offset the costs inherent to the surgical treatment.

Patients who benefit most from this type of surgery are younger and have lower BMI. Patients with DM, SAHS and HBP have better results; on the other hand arthrosis and depression have worse results.

Our results show that DRG cost might be insufficient to make a correct economic evaluation, so we recommend a method of calculating cost per patient.

SR. DIRECTOR DE DEPARTAMENTO DE CIRUGÍA Y ESPECIALIDADES MÉDICO-QUIRÚRGICAS SR. PRESIDENTE DE LA COMISIÓN ACADÉMICA DEL PROGRAMA DE DOCTORADO EN INVESTIGACIÓN EN CIRUGÍA Y ESPECIALIDADES MÉDICO-QUIRÚRGICAS 
Son muchas las personas a las que debo y quiero agradecer su colaboración, sin las cuales este proyecto no habría tenido ni sentido ni fin, con especial mención a los que más me han ayudado.

En primer lugar quiero expresar mi agradecimiento al Profesor Dr. Juan José González González, iniciador de la cirugía bariátrica en Asturias, ya que sin su motivación, enseñanza y supervisión incansable, no habría sido posible realizar este trabajo.

A todo el personal sanitario implicado en el tratamiento de la obesidad en Asturias, ya que fruto del trabajo en conjunto es posible realizar la cirugía bariátrica. Especialmente al equipo quirúrgico que tantas horas compartimos bajo las lámparas, y a la Dra. Lourdes Sanz por todo el buen trabajo, tiempo y cariño dedicado a esta Unidad y a sus pacientes.

A la doctora en Economía Dạ. Josefina Alonso Fernández, del Servicio de Contabilidad de Gestión del HUCA, por el esfuerzo realizado y la meticulosidad en su trabajo, sin duda un tiempo generosamente prestado.

A D. Ángel Mones, responsable de la Biblioteca del Hospital Vital ÁlvarezBuylla, por todo el apoyo de documentación que precisa un proyecto como este, y por su repuesta siempre inmediata.

Al Dr. Jose Ignacio Rodríguez García por su estímulo y ayuda, y por recoger el testigo de dirigir esta tesis.

A los miembros del Tribunal por su disposición y facilidades.

A mis padres, una referencia constante, por todo lo que tan generosamente me habéis dado. A mi madre por enseñarme el fascinante mundo de la gestión, y a mi padre por enseñarme todo lo médico que puede ser un cirujano.

A mis hijos, Jose Luis, Alejandro y Carmen, por dar sentido a las cosas, y siempre a Laura. 

ÍNDICE 



\section{ÍNDICE}

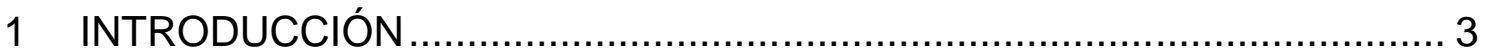

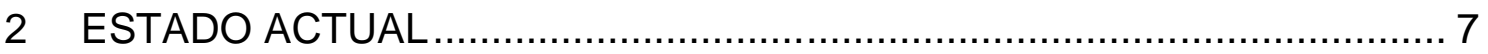

2.1 Obesidad, la epidemia del siglo XXI ............................................. 7

2.2 Evolución de la cirugía bariátrica ................................................... 10

2.3 Entendiendo la cirugía bariátrica ................................................... 17

2.3.1 Índice de masa corporal y obesidad ........................................ 17

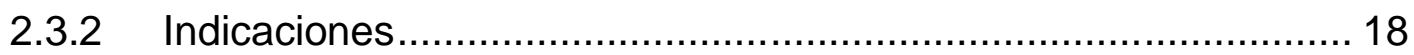

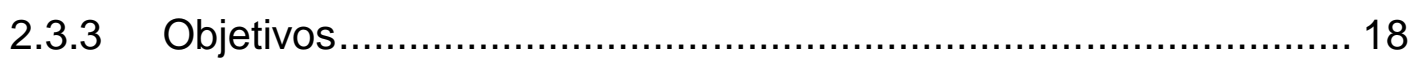

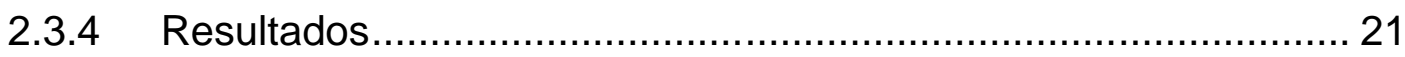

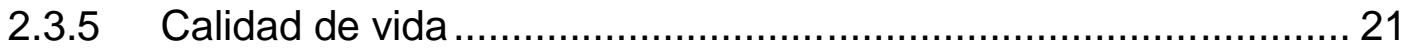

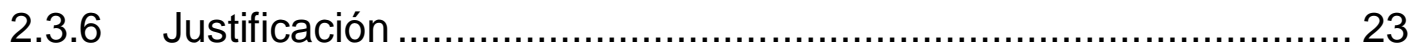

2.3.7 Cirugía laparoscópica ....................................................... 24

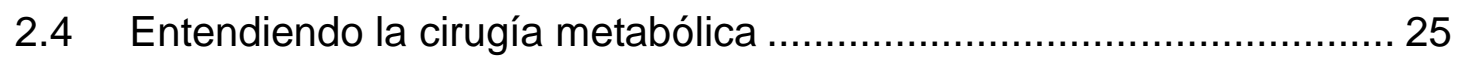

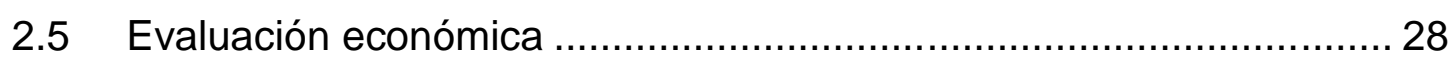

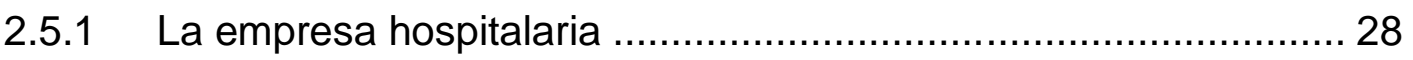

2.5.2 Análisis coste efectividad en la planificación sanitaria ................... 28

2.5.3 Normas de decisión para el análisis coste-efectividad ................. 30

2.5.4 Coste-efectividad y cirugía bariátrica ........................................... 31

2.5.5 Evaluación del gasto hospitalario ……………………….......... 34

2.5.6 Los sistemas de clasificación de pacientes (SCP)...................... 35

2.5.7 Grupos relacionados por el diagnóstico...................................... 36

2.5.8 Asignación de costes directos al paciente .................................. 39

2.6 Gasto y contexto sanitario ................................................................ 40

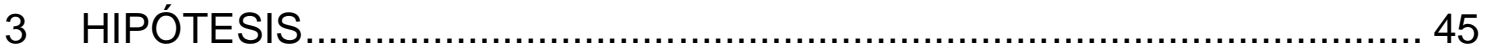

4 OBJETIVOS

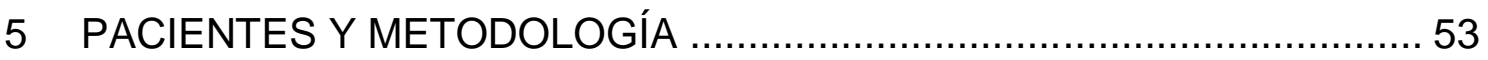

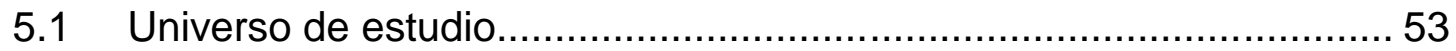

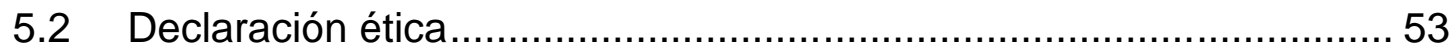

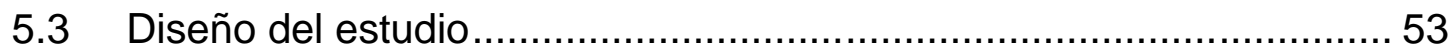




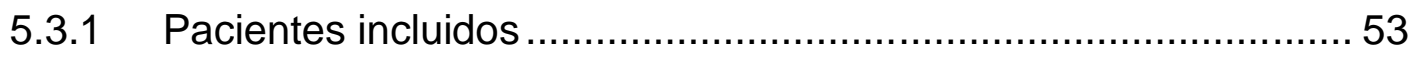

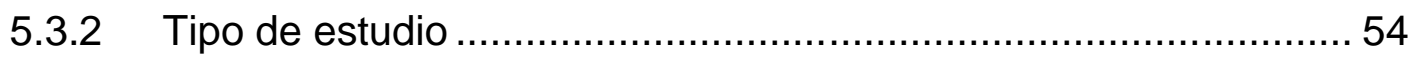

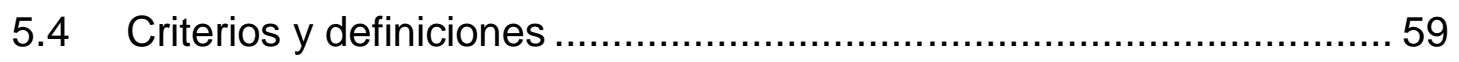

5.4.1 Criterios de inclusión .............................................................. 59

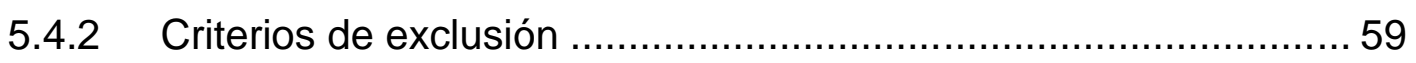

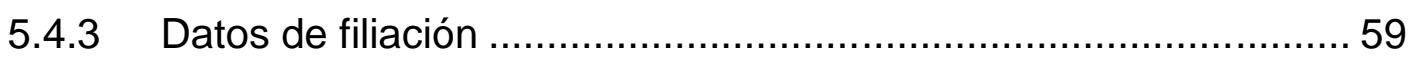

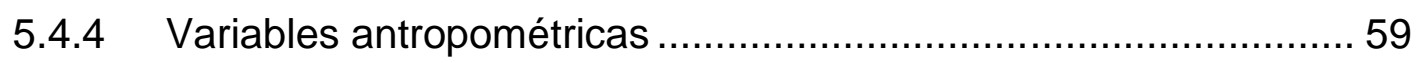

5.4.5 Comorbilidades relacionadas con la obesidad. ........................... 60

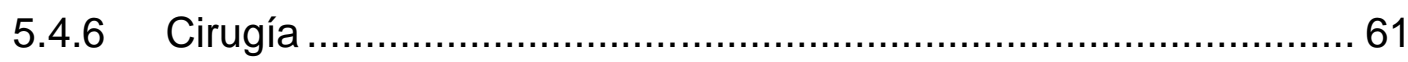

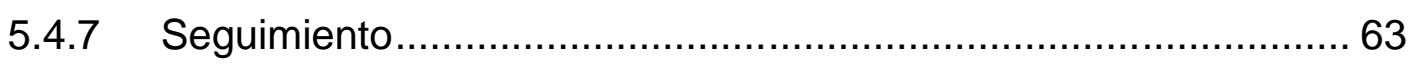

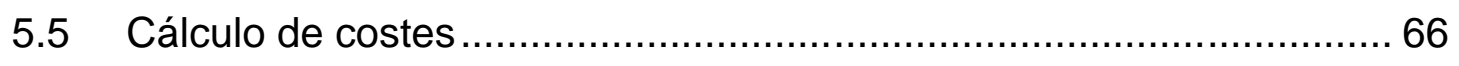

5.5.1 Contabilidad analítica mediante GRD .......................................... 66

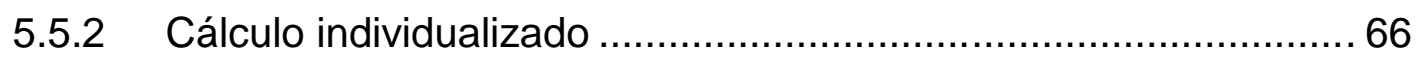

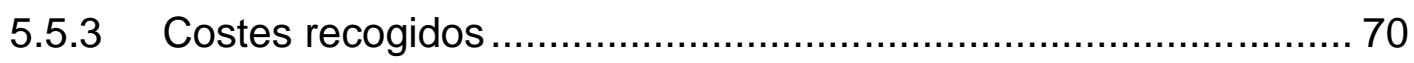

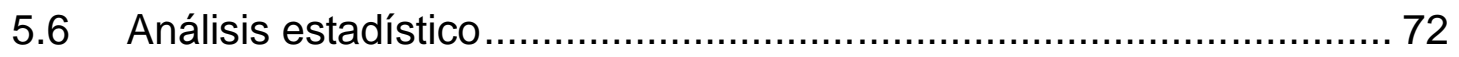

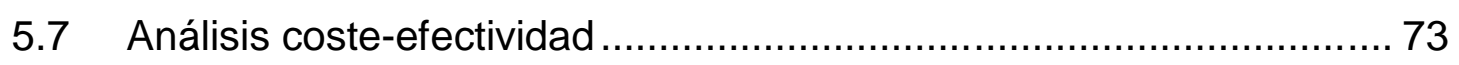

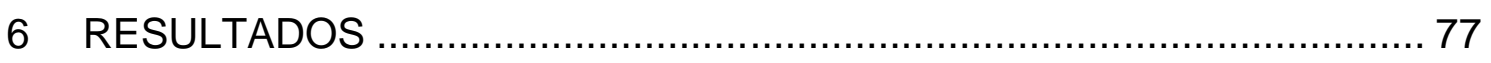

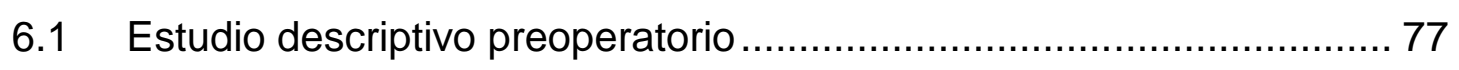

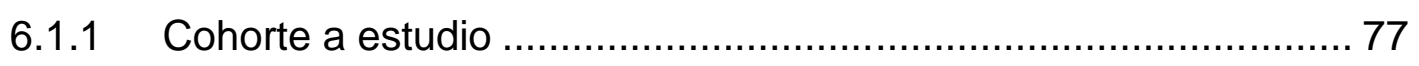

6.1.2 Características de la cohorte ................................................. 78

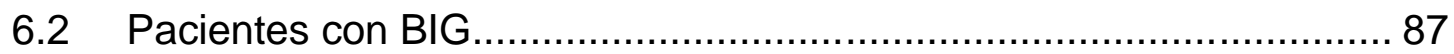

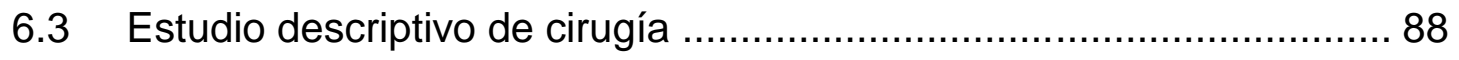

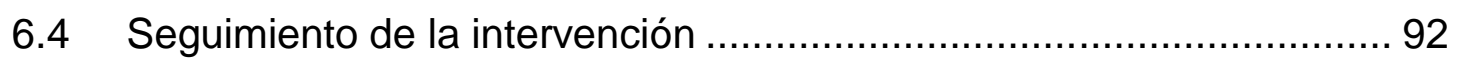

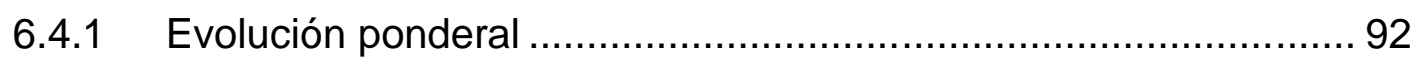

6.4.2 Modificación de fármacos tras la cirugía por patologías ................ 95

6.4.3 Cálculo del ahorro en fármacos ................................................. 102

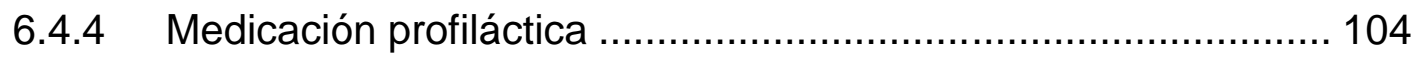

6.4.5 Cuestionario de calidad de vida BAROS .................................. 105

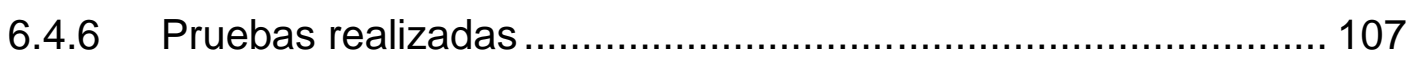

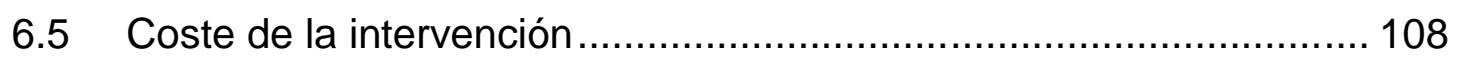

6.5.1 Contabilidad analítica mediante GRD .................................... 108

6.5.2 Cálculo individualizado ....................................................... 109

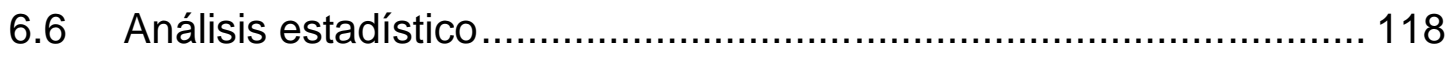


6.6.1 Pacientes con DM.............................................................. 123

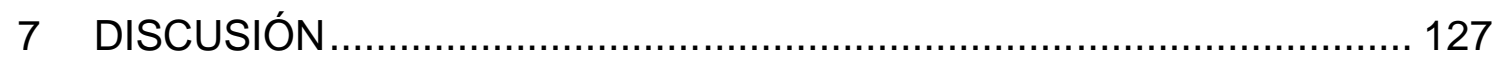

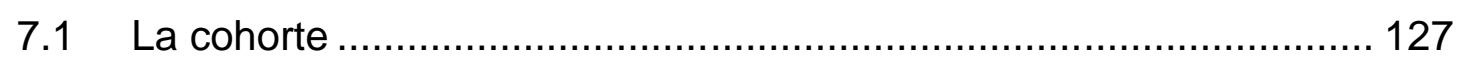

7.1.1 Parámetros demográficos y antropométricos ........................... 127

7.1.2 Comorbilidades relacionadas con la obesidad ........................ 127

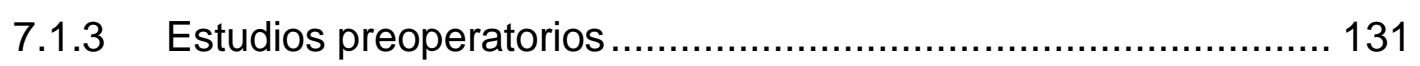

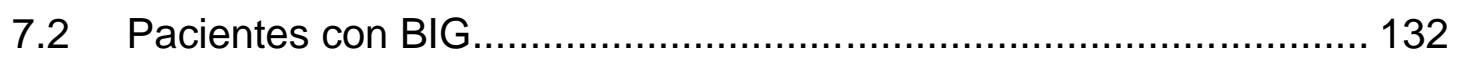

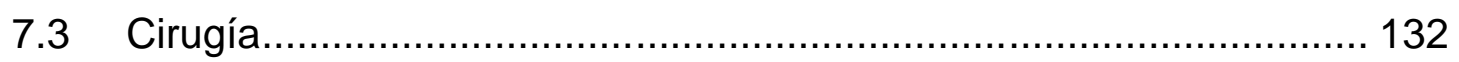

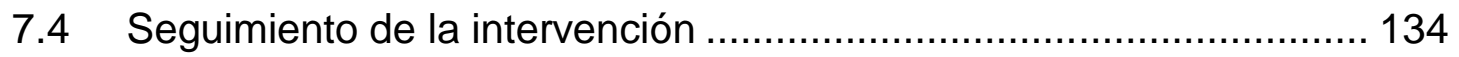

7.4.1 Evolución ponderal ............................................................ 134

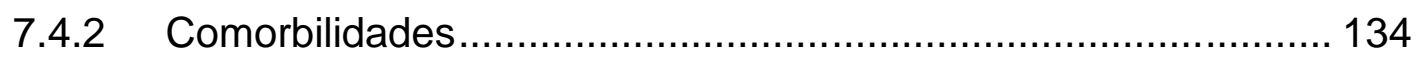

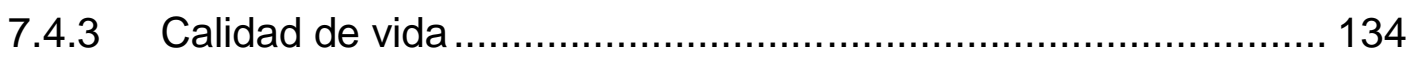

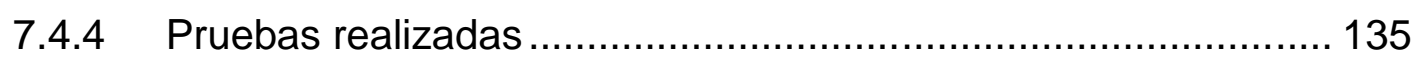

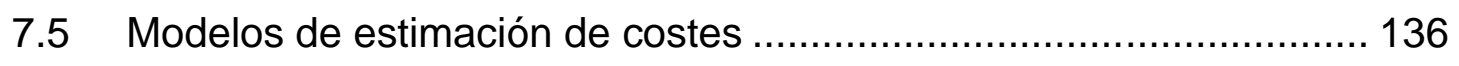

7.6 Evaluación económica ..................................................................... 138

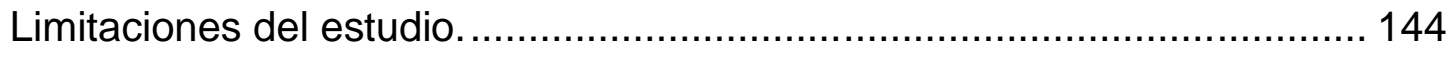

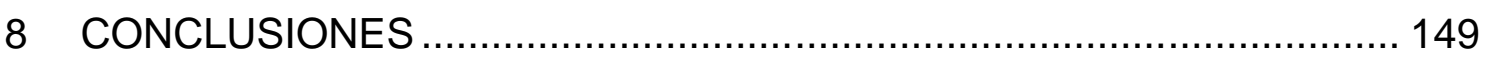

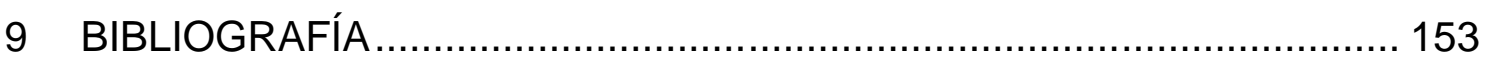






\section{ABREVIATURAS}

AA: Asa alimentaria

ABP: Asa biliopancreática

AC: Asa común

ACB: Análisis coste-beneficio

ACE: Análisis coste-efectividad

ACU: Análisis coste-utilidad

ACV: Accidente cerebrovascular

AGB: Adjustable gastric band

ASMBS: American Society for Metabolic and Bariatric Surgery

AVAC: Año de vida ajustado por calidad

BAROS: Bariatric analysis and reporting outcome system

BG: Banda gástrica

BIG: Balón intragástrico

BPD/DS: Biliopancreatic diversion / duodenal switch

BPG: Bypass gástrico

CB: Cirugía bariátrica

CD: Cruce duodenal

CE: Coste-efectividad

CEEA: Circular end-to-end anastomosis

CM: Cirugía metabólica

CMBD: Conjunto mínimo básico de datos

CPAP: Continuous positive airway pressure

CV: Calidad de vida

DBP: Derivación biliopancreática

DE: Desviación estándar 
DL: Dislipemia

DM: Diabetes mellitus

DMT2: Diabetes mellitus tipo 2

DRG: Diagnosis related group

ECV: Enfermedad cardiovascular

EGD: Estudio gastroduodenal

ERGE: Enfermedad por reflujo gastroesofágico

FDA: Food and Drug Administration

FID: Federación Internacional de Diabetes

GFH: Grupos funcionales homogéneos

GRD: Grupos relacionados por el diagnóstico

GRDR: Grupos relacionados por el diagnóstico refinados

GV: Gastrectomía vertical

GVA: Gastroplastia vertical anillada

HTA: Hipertensión arterial

HUCA: Hospital Universitario Central de Asturias

IHQ: Infección de herida quirúrgica

IMC: Índice de masa corporal

ITU: Infección del tracto urinario

LEQ: Lista de espera quirúrgica

LGS: Ley General de Sanidad

M: Media

MHO: Metabolically healthy but obese

MiniGB: Mini-gastric bypass

MIR: Médico interno residente

MLIC: Metropolitan Life Insurance Company

MSSSI: Ministerio de Sanidad, Servicios Sociales e Igualdad 
NHS: National Health Service

NICE: National Institute for Health and Care Excellence

OCDE: Organización para la Cooperación y el Desarrollo Económico

OIB: Oficina de Investigación Biosanitaria

OM: Obesidad mórbida

OMS: Organización Mundial de la Salud

PEIMCP: Porcentaje de exceso de índice de masa corporal perdido

PMC: Patient management categories

PSP: Porcentaje de sobrepeso perdido

RYGB: Roux-en-Y gastric bypass

SAHS: Síndrome de apnea e hipopnea del sueño

SCP: Sistema de clasificación de pacientes

SECO: Sociedad Española de Cirugía de la Obesidad

SEEDO: Sociedad Española para el Estudio de la Obesidad

SESPA: Servicio de Salud del Principado de Asturias

SG: Sleeve gastrectomy

SM: Síndrome metabólico

SNS: Sistema Nacional de Salud

TA: Tensión arterial

TC: Tomografía computarizada

TVP: Trombosis venosa profunda

UCH: Unidad de complejidad hospitalaria 




\section{TABLAS}

Tabla 1. Criterios SEEDO para definir la obesidad en grados según el IMC en adultos

Tabla 2. Resultados en \% según el tipo de cirugía: Banda gástrica (BG), Gastrectomía vertical (GV) y Bypass gástrico (BPG). Tomados de Dogan

Tabla 3. Definición de síndrome metabólico por la Federación Internacional de Diabetes

Tabla 4. Gasto sanitario según el agente de financiación (en \%). Fuente MSSSI

Tabla 5. Gasto sanitario público en España y Asturias

Tabla 6. Calibrado de asas según IMC

Tabla 7. Revisiones y pruebas del seguimiento de cirugía bariátrica

Tabla 8. Evaluación final de resultados según el cuestionario BAROS

Tabla 9. Características generales de la serie

Tabla 10. Características de la población en función del género

Tabla 11. Características de la población en función del IMC de la primera consulta

Tabla 12. Características de la población en función del IMC preoperatorio

Tabla 13. Número de fármacos por patología

Tabla 14. Evolución ponderal durante el periodo con BIG, media (DE) / rango

Tabla 15. Características de la población en función del abordaje quirúrgico

Tabla 16. Complicaciones postoperatorias. $99 / 307$ casos $(32,2 \%)$

Tabla 17. Causas de reintervención según abordaje del BPG. 14/307 pacientes

Tabla 18. Evolución ponderal de toda la serie, media (DE) / rango 
Tabla 19. Evolución postoperatoria de la HTA

Tabla 20. Evolución postoperatoria de la DM

Tabla 21. Evolución postoperatoria de la DL

Tabla 22. Evolución postoperatoria del SAHS

Tabla 23. Evolución postoperatoria de la artrosis

Tabla 24. Evolución postoperatoria de la depresión

Tabla 25. Número de fármacos por paciente de la serie completa (307), por grupos de peso según su índice de masa corporal (IMC), en primera consulta

Tabla 26. Número de fármacos por paciente que toman fármacos (210), por grupos de peso según su índice de masa corporal (IMC), en primera consulta

Tabla 27. Pacientes con suplementos habituales en las sucesivas revisiones

Tabla 28. Suplementos no habituales en las sucesivas revisiones

Tabla 29. Definición global de su situación respecto a antes de la cirugía

Tabla 30. Resultado del cuestionario BAROS por apartados

Tabla 31. Clasificación en grupos de porcentaje de peso perdido (PSP), según el BAROS

Tabla 32. Evolución de comorbilidades según el cuestionario BAROS $(n=238)$

Tabla 33. Evaluación final del cuestionario BAROS, si presentaba o no comorbilidades previas

Tabla 34. Costes en función del GRD al alta

Tabla 35. Costes de personal para el cálculo individualizado

Tabla 36. Coste en euros de los fármacos empleados durante el ingreso estándar

Tabla 37. Material quirúrgico empleado en el BPG laparoscópico 
Tabla 38. Material quirúrgico empleado en reintervenciones

Tabla 39. Costes de pruebas de imagen durante el ingreso

Tabla 40. Costes de estudios y consultas perioperatorias

Tabla 41. Inversión inicial para iniciar el programa de cirugía bariátrica

Tabla 42. Comparación de costes por abordaje y peso, recogido en la media en euros

Tabla 43. Nivel de significación (p) de las pruebas estadísticas utilizadas

Tabla 44. Medias de coste-efectividad de la cirugía abierta vs laparoscópica

Tabla 45. Características de los pacientes diabéticos

Tabla 46. Coste de fármacos antihipertensivos en España. Tomado de GarcíaRuíz

Tabla 47. Gasto sanitario español, costes directos por patología: HTA y DM

Tabla 48. Estudios de amortización de la cirugía bariátrica 

Figura 1. Evolución de la obesidad en países de la OCDE. Tomada de www.oecd.org

Figura 2. Esquema de la derivación biliopancreática y del cruce duodenal

Figura 3. Esquema de la banda gástrica

Figura 4. Esquema de la gastrectomía vertical

Figura 5. Esquema del bypass gástrico actual

Figura 6. Esquema del primer bypass gástrico

Figura 7. Esquema de la gastroplastia vertical anillada

Figura 8. Evolución de la cirugía bariátrica en EEUU/Canada (izquierda) y Europa (derecha). AGB: Banda gástrica, SG: Gastrectomía vertical, RYGB: Bypass gástrico, BPD/DS: Cruce duodenal, MiniGB: Minibypass gástrico. Tomado de Buchwald

Figura 9. Cuestionario BAROS

Figura 10. Lipotoxicidad como causa de diabetes mellitus y síndrome metabólico. Tomado de Pok

Figura 11. Número de cirugías bariátricas en Europa. Tomado de Borisenko

Figura 12. Formulario de primera consulta de cirugía bariátrica

Figura 13. Formulario de colocación y seguimiento del balón intragástrico

Figura 14. Formulario de alta de cirugía bariátrica

Figura 15. Protocolo de recogida de datos

Figura 16. Bypass gástrico. Tipos de asas para su calibrado

Figura 17. Formulario de seguimiento de cirugía bariátrica 
Figura 18. Diagrama de la selección de pacientes incluidos en el estudio

Figura 19. Diagrama de cajas por edad y sexo

Figura 20. Comorbilidades principales relacionadas con la obesidad

Figura 21. Número de fármacos para la HTA antes de la cirugía

Figura 22. Número de fármacos para la DM antes de la cirugía

Figura 23. Número de fármacos para la DL antes de la cirugía

Figura 24. Número de fármacos para la artrosis antes de la cirugía

Figura 25. Número de fármacos para la depresión antes de la cirugía

Figura 26. Evolución ponderal por porcentaje de sobrepeso perdido

Figura 27. Evolución ponderal por porcentaje de exceso de IMC perdido

Figura 28. Porcentaje de sobrepeso perdido en el seguimiento, según abordaje

Figura 29. Porcentaje de sobrepeso perdido en el seguimiento, según BIG previo

Figura 30. Número de fármacos para la HTA en el seguimiento

Figura 31. Número de fármacos para la DM en el seguimiento

Figura 32. Número de fármacos para la $\mathrm{DL}$ en el seguimiento

Figura 33. Porcentaje de pacientes que utilizan la CPAP en el seguimiento

Figura 34. Número de fármacos para la artrosis en el seguimiento

Figura 35. Número de fármacos para la depresión en el seguimiento

Figura 36. Número de fármacos para las principales comorbilidades, en el seguimiento

Figura 37. Evolución de la media de consumo de fármacos en el seguimiento 


\section{INTRODUCCIÓN}





\section{INTRODUCCIÓN}

La obesidad y sus comorbilidades son un problema económico y de salud pública de gran trascendencia. En Asturias, la prevalencia de la obesidad es del $18,7 \%$ y del sobrepeso $38,5 \%$ (1), cifras por encima de la media en España (17 y $36,7 \%$ respectivamente). Sus consecuencias personales se manifiestan tanto en la esperanza como en la calidad de vida. A nivel social la pérdida de movilidad y productividad suponen un coste económico adicional para la sociedad.

Comprobado el fracaso de los tratamientos médico, farmacológico y conductual de la obesidad mórbida establecida $(2,3)$, el único razonablemente eficaz es la cirugía bariátrica y dentro de ésta, el bypass gástrico (BPG) es la intervención de referencia de todas las disponibles en este momento $(4,5)$. Sus resultados son satisfactorios a corto y largo plazo en cuanto a pérdida de peso (mayor del $50 \%$ de sobrepeso perdido a cinco años), resolución de las comorbilidades y mejoría de la calidad de vida, las tres categorías principales que dan sentido al tratamiento de la obesidad mórbida (6-8), pero ¿a qué coste económico?.

La demanda socio-sanitaria es creciente, no solo por la epidemia de obesidad que vivimos, sino también porque los buenos resultados clínicos y bajas complicaciones de la intervención animan a la población a elegir esta opción terapéutica, a pesar de ser una cirugía compleja y tratarse de pacientes en muchos casos de alto riesgo quirúrgico.

El coste de la cirugía bariátrica no ha sido bien estudiado hasta el momento en nuestro país y los que lo han realizado (9) han dado por buenos los métodos clásicos de evaluación económica hospitalaria, presumiblemente insuficientes. De la misma forma no existe hasta la fecha una respuesta unánime a la pregunta de si el tratamiento quirúrgico de la obesidad es coste-efectivo, entre otras razones por el elevado número de variables que entran en juego a la hora de hacer una evaluación de estas características, entre las que cabe citar ahorro de consultas o fármacos para el control de las comorbilidades, o las bajas laborales. 
Las restricciones presupuestarias actuales y las que se intuyen en el futuro hacen de gran interés el conocimiento del coste real de la cirugía bariátrica en nuestro medio, en este momento el BPG, y los beneficios conseguidos con ella. En la medida que podamos cuantificarlos, justificaremos en términos económicos su aplicación. 
ESTADO ACTUAL 



\section{ESTADO ACTUAL}

\subsection{Obesidad, la epidemia del siglo XXI}

Según la definición de la Organización Mundial de la Salud (OMS): "La obesidad y el sobrepeso se definen como una acumulación anormal o excesiva de grasa que puede ser perjudicial para la salud".

La obesidad es una enfermedad en la que existe un aumento de los depósitos corporales de energía en forma de grasa, lo que condiciona un incremento del tejido adiposo. Es la enfermedad más frecuente del mundo occidental. La etiopatogenia es compleja y heterogénea, en algunos casos es posible identificar una causa primordial (endocrinológica, genética, fármacos,..), pero en la mayoría de ellos no. Se sabe desde hace tiempo que tanto en la génesis como en el mantenimiento de la obesidad influyen tanto factores biológicos como ambientales. Entre los factores biológicos se cita la predisposición genética; se han identificado múltiples regiones en el ADN relacionadas con la obesidad, la diabetes mellitus (DM) y el síndrome metabólico (SM) (10), por desgracia con limitada aplicación clínica por el momento, pero con un papel protagonista todavía por desempeñar en áreas como la identificación de individuos de riesgo, para su prevención (11). Entre los factores ambientales, derivados de nuestro estilo de vida, los cambios en la dieta y el sedentarismo, lo que se conoce como the big two $(12,13)$ son los más importantes. El papel del sedentarismo es reconocido de manera unánime y la recomendación de incrementar el ejercicio físico aparece en múltiples guías para la prevención de enfermedades crónicas (14), ya que aumenta el gasto energético total además de modificar la composición corporal, aumentando el porcentaje de masa muscular y disminuyendo la masa grasa. En cuanto a los cambios en la dieta, en el último siglo existe una mayor disponibilidad de alimentos y un trasfondo cultural que estimula la ingesta de los mismos más allá de lo estrictamente necesario, así como un cambio en su composición, que en general originan una menor sensación de saciedad y por tanto un mayor consumo calórico. 
En la clínica una forma práctica y simple de medir la obesidad es la estimación del índice de masa corporal (IMC), esto es el peso de una persona en kilogramos dividido por el cuadrado de la talla en metros. Una persona con un IMC igual o superior a 30 es considerada obesa y con un IMC igual o mayor de 25 se habla de sobrepeso. El sobrepeso y la obesidad son factores de riesgo para numerosas enfermedades crónicas, entre las que se incluyen la diabetes mellitus tipo 2 (DMT2), enfermedad cardiovascular (ECV) y renal, accidente cerebrovascular (ACV), artrosis, reflujo gastroesofágico, trastornos motores gastrointestinales, síndrome de apnea e hipopnea del sueño (SAHS), algunos tipos de cáncer, esteatohepatitis no alcohólica, depresión y otros (15-21). Todos ellos influyen negativamente en la calidad y esperanza de vida.

La obesidad es la segunda causa de muerte evitable tras el tabaco, y representa más del $15 \%$ de la mortalidad atribuible en nuestro país (22). El riesgo de mortalidad se incrementa a medida que lo hace el IMC (23), y aquellos con obesidad mórbida rebajan en 10 años su expectativa de vida (24), similar al efecto de haber fumado siempre. De otra parte, el $90 \%$ de los casos de DMT2 (25), el 50\% de los eventos coronarios (26) y un incremento del riesgo atribuido del 3 al 9\% de algunos tumores malignos (27): endometrio, mama en mujeres postmenopáusicas, colon,... se relacionan en mayor 0 menor grado con el sobrepeso y la obesidad. En un estudio realizado a profesionales de la salud se calculó que un $30 \%$ de los cánceres de colon estaban relacionados con el sobrepeso o la obesidad (28).

Si bien el sobrepeso y la obesidad tiempo atrás eran considerados un problema propio de los países de ingresos altos, en la actualidad ambos trastornos han aumentado en los países de ingresos bajos y medianos (29), en particular en los entornos urbanos. En los países en desarrollo con economías emergentes (clasificados por el Banco Mundial en países de ingresos bajos y medianos) el incremento porcentual del sobrepeso y la obesidad en los niños ha sido un $30 \%$ superior al de los países desarrollados. La mayoría de la población mundial vive en países donde el sobrepeso y la obesidad se cobran más vidas de personas que la insuficiencia ponderal (30). 
El número de personas con sobrepeso y obesidad en el mundo aumentó de 857 millones a 2100 millones en los últimos 33 años. Un análisis realizado en 188 países entre 1980 y 2013 determinó que el incremento de la obesidad a nivel global fue de un $27,5 \%$ en adultos y de un $47,1 \%$ en niños. Más del $50 \%$ de los 671 millones de personas obesas en el mundo viven en solo 10 países: Estados Unidos (más de 13\%), China y la India (15\% combinado), Rusia, Brasil, México, Egipto, Alemania, Pakistán e Indonesia (29).

En España de cada 100 adultos 17 padecen obesidad y 37 presentan sobrepeso, dicho de otra manera más de la mitad de la población española mayor de 18 años padece al menos sobrepeso. La obesidad ha aumentado del 7,4 al 17\% en los últimos 25 años. Hay autores que sitúan esta cifra en torno al 23\% (31). De cada 10 niños y adolescentes de 2 a 17 años, dos tienen sobrepeso y uno obesidad. El $41,3 \%$ de la población se declara sedentaria, algo menos de la mitad de las mujeres $(46,6 \%)$ y más de un tercio de los hombres $(35,9 \%)(32)$.

Países de nuestro entorno cultural más cercano, como Italia (10\%), Austria $(12,4 \%)$, Francia (12,9\%) o Alemania (14,2\%) tienen índices de obesidad adulta más bajos que España, mientras que países como Grecia (19,6\%), Australia $(28,3 \%)$ o Estados Unidos (32,5\%) tienen porcentajes (figura 1) de obesidad adulta superiores (33).

Si bien es un cálculo complejo, se estima que España destina el $7 \%$ del gasto sanitario anual al tratamiento del sobrepeso y la obesidad, alrededor de 5000 millones de euros al año (34), aunque esta cifra puede estar infravalorada, ya que la tendencia es al aumento del gasto en estos pacientes (35). A nivel mundial los costes directos de la obesidad se calcula que representan entre el 2 y el $7 \%$ del gasto sanitario (36). 


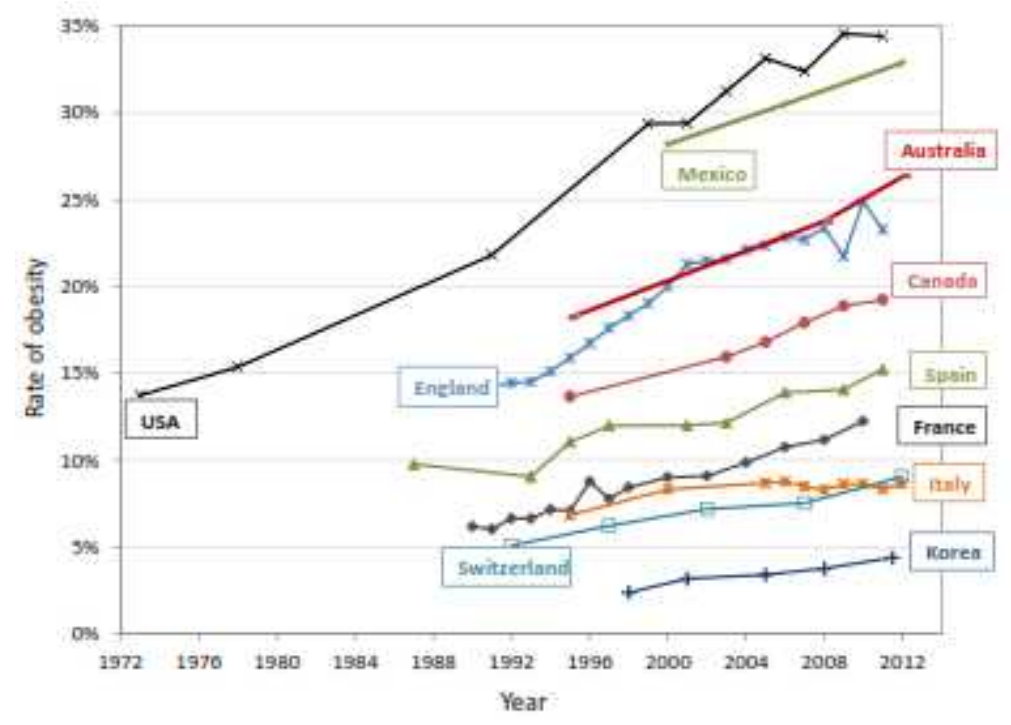

Figura 1. Evolución de la obesidad en países de la OCDE. Tomada de www.oecd.org

\subsection{Evolución de la cirugía bariátrica}

La primera actuación quirúrgica enfocada a tratar la obesidad de la que se tiene constancia tuvo lugar en España. Sancho I Rey de León (935-966 d.C), reinados (956-958 y 960-966) era orgulloso, fatuo, prepotente y monstruosamente obeso, lo que le impedía montar a caballo, manejar las armas e incluso caminar, por lo que perdió el trono a manos de los nobles y hubo de huir a Navarra bajo la protección de su abuela la Reina Toda (37). Dispuesta a recuperar el trono para su nieto la Reina Toda viajó con Sancho a Córdoba para que Hasday ibn Shaprut, famoso médico judío de la Corte de Abderramán III lo sometiese a tratamiento. El tratamiento, médico-quirúrgico y de seis meses de duración, consistió en suturarle los labios y alimentarlo con una paja a base quizá de teriaca un polifármaco que contenía un número variable de ingredientes, a veces más de setenta, entre los que destacaba el opio cuyos efectos colaterales hacen perder peso $(38,39)$. Sancho volvió cabalgando a León y con la ayuda del Reino de Navarra y el ejército de Abderramán III reconquistó el trono. Por desgracia, en una expedición a Galicia 
y Portugal falleció envenenado con una manzana que le ofreció al parecer un noble portugués, en Noviembre de 966 (37), pero curado de la obesidad (39).

\section{La época moderna}

El término bariátrica fue adoptado hacia 1965 y consta del prefijo griego baro $=$ peso y el sufijo iatros = los que practican la medicina. Asociado a la palabra cirugía se refiere a la cirugía de la obesidad (40).

En poco más de medio siglo la cirugía bariátrica $(\mathrm{CB})$ se ha convertido en una de las disciplinas de la cirugía del aparato digestivo con mayor desarrollo y diversidad de intervenciones. Suelen agruparse en tres tipos, en función del mecanismo fundamental que utilizan para inducir la pérdida de peso: malabsortivas como la derivación biliopancreática (DBP) y cruce duodenal (CD) (figura 2), restrictivas como la banda gástrica (BG) (figura 3) ○ la gastrectomía vertical (GV) (figura 4) y mixtas como el bypass gástrico (BPG) (figura 5). Con perspectiva histórica podemos afirmar que son seis los procedimientos principales dentro de esta disciplina (41), algunos de ellos ya en desuso, bien por pérdida de eficacia a largo plazo o bien por sus secuelas. Otros de implantación reciente aunque prometedores deben superar la prueba del tiempo.
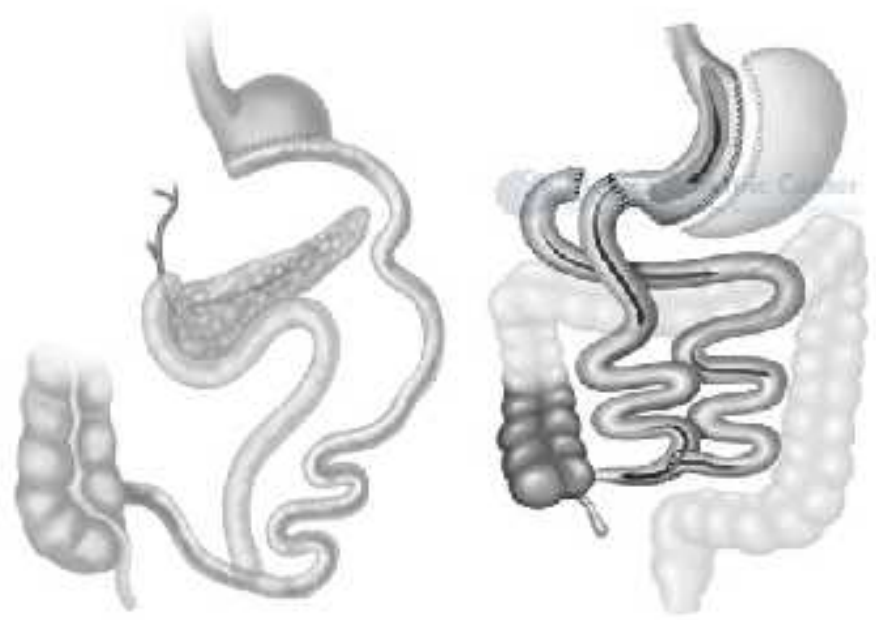

Figura 2. Esquema de la derivación biliopancreática y del cruce duodenal 


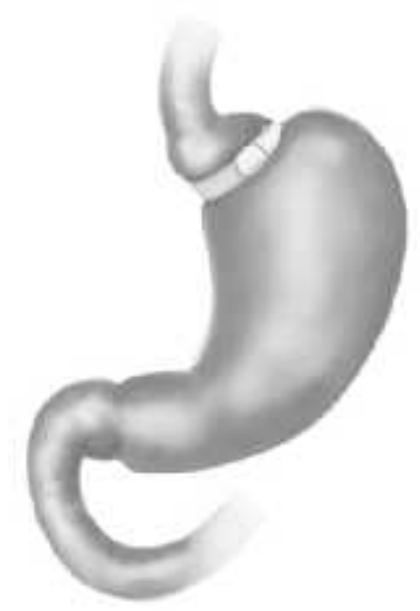

Figura 3. Esquema de la banda gástrica

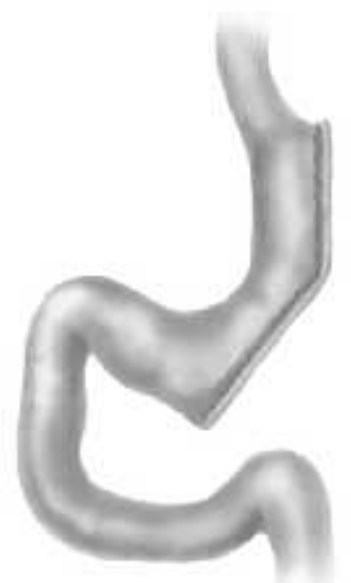

Figura 4. Esquema de la gastrectomía vertical 


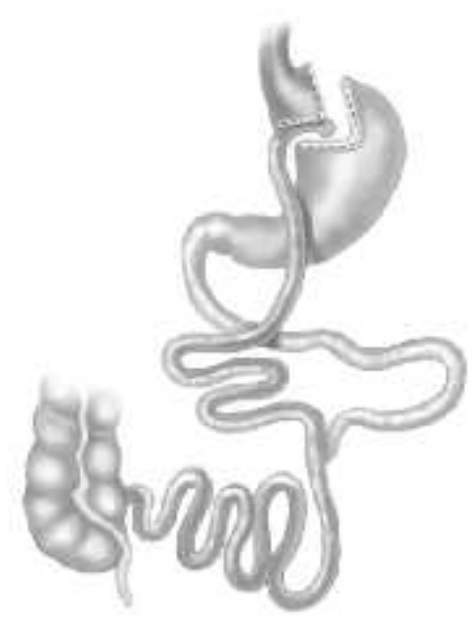

Figura 5. Esquema del bypass gástrico actual

El tratamiento quirúrgico de la obesidad se desarrolló a partir de la pérdida de peso observada en pacientes a los que se les realizaban resecciones intestinales o gástricas. Si seguimos un orden cronológico de las técnicas de CB debemos señalar que la primera intervención para tratar la obesidad fue realizada por Henrikson en 1952 (42), mediante una resección masiva de intestino delgado. Esta cirugía, obviamente irreversible, se siguió de varios tipos de bypass de intestino delgado sin resección del intestino excluido, por tanto reversibles. En el primero descrito (bypass yeyunocólico), basado en observaciones en animales, el yeyuno proximal se anastomosó a la mitad del colon transverso con el propósito de en un segundo tiempo, conseguido el peso deseado, restablecer el tránsito intestinal. El bypass yeyunoileal, descrito por primera vez en la Universidad de Minnesota por Kremen, dejaba sólo $40 \mathrm{~cm}$ de yeyuno proximal en circulación y los unía al íleon a $10 \mathrm{~cm}$ de la válvula ileocecal (43). Se trata de una intervención eminentemente malabsortiva, eficaz en pérdida de peso y duradera en el tiempo, que desapareció de los recursos quirúrgicos en obesidad por sus complicaciones (44), tan graves como la insuficiencia hepática, el síndrome de intestino corto y el sobrecrecimiento bacteriano en el intestino excluido, en una época en la que los tratamientos farmacológicos sustitutivos eran desconocidos. Mason, padre de la $\mathrm{CB}$, publicó (45) en el año 1966 el primer BPG (figura 6); diez años después, de nuevo en la Universidad de Minnesota evolucionará hacia el primer BPG vertical con 
gastroyeyunostomía en $\mathrm{Y}$ de Roux, el que se realiza en la actualidad (figura 5). En 1994 Wittgrove publicaría los primeros casos con abordaje laparoscópico (46).

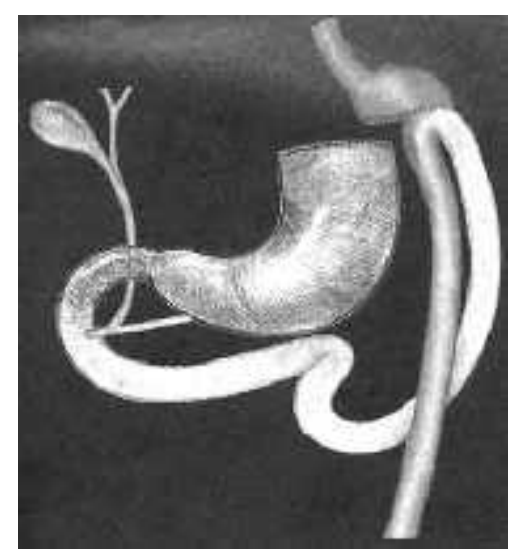

Figura 6. Esquema del primer bypass gástrico

En 1982 Mason describió la gastroplastia vertical anillada (GVA) (figura 7) con una malla de Marlex, procedimiento puramente restrictivo, que rivalizó con el BPG en la década de 1980 y 1990. Desafortunadamente la reganancia de peso, así como las complicaciones y necesidad frecuente de otra cirugía (47) terminó por hacerla desaparecer, favorecido además por la entrada en escena sobre todo en Europa y Australia de la BG, procedimiento también restrictivo y de colocación sencilla por vía laparoscópica. Siguiendo una trayectoria similar la expansión actual de la GV, permite augurar que la $B G$ se trata de un procedimiento en vías de extinción, con unas cifras en EEUU por debajo del $5 \%$ en los últimos años $(48,49)$.

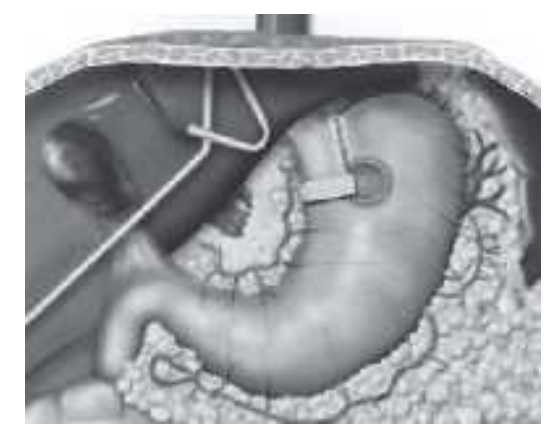

Figura 7. Esquema de la gastroplastia vertical anillada 
Nicola Scopinaro, de Génova, en 1979 (50) busca alcanzar la pérdida de peso del bypass yeyunoileal, sin sus complicaciones nutricionales y la diarrea; propone una intervención entendida como mixta aunque con claro predominio malabsortivo, se trata de la DBP. Esta intervención resultó, en su travesía atlántica, en el cruce duodenal (CD) descrito por Hess en 1998 (51). En manos expertas ambas intervenciones consiguen la mejor pérdida de peso mantenida, así como resolución de las comorbilidades, de todas las técnicas de cirugía bariátrica. El problema radica en las secuelas digestivas, nutricionales y quirúrgicas que inducen (52), de ahí lo limitado de su indicación que en la actualidad en EEUU se encuentra por debajo del 1\% (53).

Por último encontramos la GV, que comenzó a realizarse como parte del CD en 1998 (51) y fue descrita por primera vez por Regan (54) en 2003 y popularizada por Michel Gagner como un primer tiempo hacia el CD en pacientes de alto riesgo. Es una intervención puramente restrictiva que consiste en seccionar el $80 \%$ del estómago, a expensas de la curvatura mayor gástrica, dejando un estómago tubular de un diámetro aproximado al del esófago. Es en la actualidad la técnica de CB más realizada en EEUU $(48,49,53)$, una tendencia (figura 8) que empieza a seguirse en Europa $(4,55)$ y en España $(56)$, favorecido por la sensación de ser una cirugía sencilla y de menor morbilidad, y aunque su índice de fístulas puede llegar hasta el 14\% (57), los resultados nos indican que la mortalidad y fístulas son equiparables al BPG (58). Aún no se conocen los resultados a largo plazo, pero estudios recientes apuntan que serían similares al BPG en resolución de la diabetes (59), aunque con peor disminución del riesgo cardiovascular (60), mientras que en otros estudios esto no se avala $(61,62)$. Algunos autores la consideran la técnica ideal (63) e incluso se señala para tratar complicaciones propias del BPG (64) como la úlcera marginal, el síndrome de dumping y el sobrecrecimiento bacteriano, aunque en realidad todas ellas suelen controlarse con tratamiento médico. Su futuro es impredecible, pero sí podemos intuir que puede terminar convirtiéndose en el primer tiempo de una $\mathrm{CB}$ de componente malabsortivo. Como limitaciones estarían los pacientes con reflujo y esofagitis, así como aquellos con alteraciones metabólicas graves. 

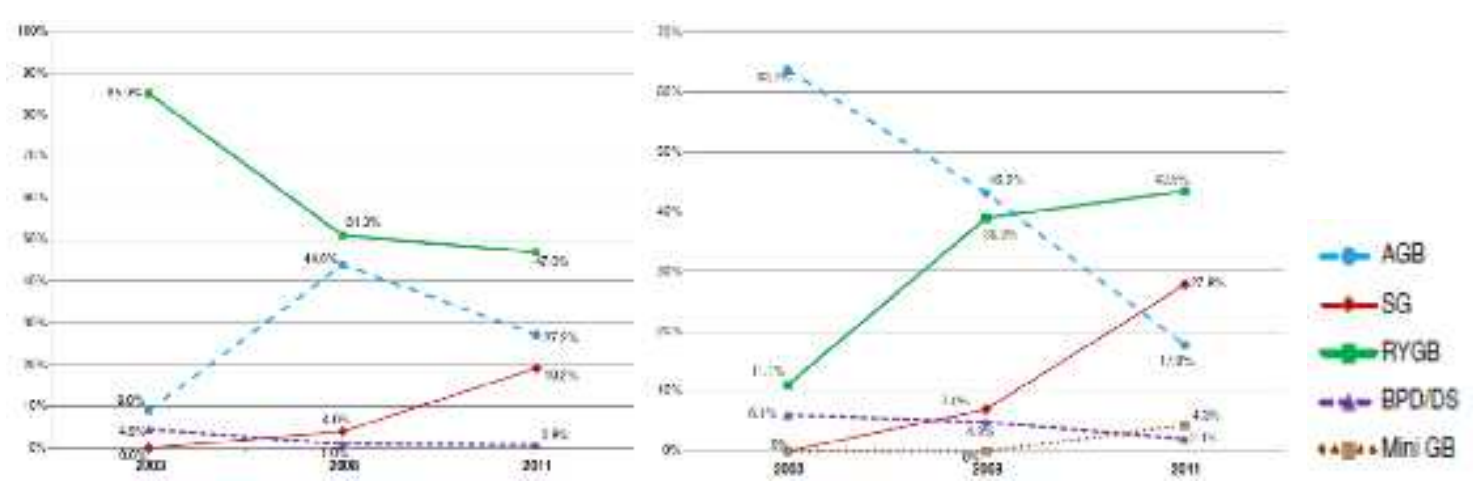

Figura 8. Evolución de la cirugía bariátrica en EEUU/Canada (izquierda) y Europa (derecha). AGB: Banda gástrica, SG: Gastrectomía vertical, RYGB: Bypass gástrico, BPD/DS: Cruce duodenal, MiniGB: Minibypass gástrico. Tomado de Buchwald (4)

Aunque todos los procedimientos mencionados logran resultados aceptables, de la misma manera todos ellos han fracasado en determinados pacientes, el BPG, avalado por medio siglo de estabilidad en un entorno especialmente dinámico, se ha convertido en el gold standard del tratamiento quirúrgico de la obesidad, siendo hasta hace muy poco la intervención más practicada en EEUU (4), debido a que ofrece una adecuada reducción de peso, sin aumentar la mortalidad ni los efectos adversos; además, reduce de manera importante la prevalencia de patologías como la hipertensión arterial y la DMT2, con un objetivo de porcentaje de exceso de peso perdido a los dos años en torno al $70 \%(65,66)$ y a los 5 años un $75 \%$ de los pacientes deberían alcanzar un tanto por ciento de exceso de peso perdido de al menos el 50\% (67), así como mantener resolución de comorbilidades (68). Aunque también es cierto que con el tiempo van aumentando los índices de fracaso y las cirugías de revisión.

En la actualidad el BPG, la GV y la BG, representan el $92 \%$ de las intervenciones de $C B$ (4) a nivel mundial. Con una estabilización del BPG en los últimos años y una evolución de la GV, en detrimento de la BG, claramente inferior y con un elevado índice de revisión pasados los dos años (66). 


\subsection{Entendiendo la cirugía bariátrica}

\subsection{1 Índice de masa corporal y obesidad}

El Índice de Masa Corporal (IMC), una medición estadística que relaciona el peso con la estatura, fue descrito por el estadístico y astrónomo belga Adolphe Quetelet en 1835 (69).

En 1959 la Metropolitan Life Insurance Company (MLIC), preocupada por su cuenta de resultados y los efectos negativos de la obesidad para su negocio, hizo los primeros intentos para definir el peso ideal, es decir el que estadística y teóricamente permite mayor longevidad para una altura y sexo determinados, y así clasificar la obesidad. En 1960 establecen que un IMC de $30 \mathrm{~kg} / \mathrm{m}^{2} \mathrm{o}$ superior debe ser considerado científicamente obesidad. La Sociedad Americana de Cirugía Bariátrica (ASMBS) (70) fija años más tarde que el IMC de $40 \mathrm{~kg} / \mathrm{m}^{2}$ o superior será catalogado como obesidad mórbida (OM).

La Sociedad Española para el Estudio de la Obesidad (SEEDO), en un documento de consenso publicado en 2007 (71), presentó algunas modificaciones a la clasificación según IMC propuesta por la OMS: rebajó el límite inferior del peso normal a $18,5 \mathrm{~kg} / \mathrm{m}^{2}$, subdividió la gama de sobrepeso en 2 categorías e introdujo un grado adicional de obesidad para los pacientes con IMC de $50 \mathrm{~kg} / \mathrm{m}^{2}$ o superior, que son tributarios de indicaciones especiales en la elección del procedimiento de CB (tabla 1).

Tabla 1. Criterios SEEDO para definir la obesidad en grados según el IMC en adultos

\begin{tabular}{|l|c|}
\hline \multicolumn{1}{|c|}{ Categoría } & Valores límite de IMC $\left.\mathbf{( k g / \mathbf { m } ^ { 2 }}\right)$ \\
\hline Peso insuficiente & $18,5-24,9$ \\
\hline Peso normal & $25-26,9$ \\
\hline Sobrepeso grado I & $27-29,9$ \\
\hline Sobrepeso grado II & $30-34,9$ \\
\hline Obesidad tipo I & $35-39,9$ \\
\hline Obesidad tipo II & $40-49,9$ \\
\hline Obesidad tipo III (mórbida) & $\geq 50$ \\
\hline Obesidad tipo IV (extrema) & \\
\hline
\end{tabular}




\subsubsection{Indicaciones}

Desde el año 1991, en conferencia de consenso, se establecieron las indicaciones de la CB mediante el IMC y comorbilidades graves (72). De tal manera que serán candidatos aquellos pacientes con $I M C \geq 40 \mathrm{~kg} / \mathrm{m}^{2}$ o con un IMC entre 35 y $40 \mathrm{~kg} / \mathrm{m}^{2}$, si se asocia a alguna comorbilidad como DMT2, hipertensión arterial o dislipemia (17-20,72); pero también asumiendo ciertos requisitos de estabilidad psicológica y compromisos para el seguimiento por parte del paciente para garantizar un éxito a largo plazo (73). Cada día aumenta la evidencia por la que en los pacientes asiáticos debería existir una disminución de $2,5 \mathrm{~kg} / \mathrm{m}^{2}$ por cada categoría de indicación, por la alta prevalencia de obesidad troncular de esa población (74).

Esta aproximación terapéutica debe estar acompañada de un equipo multidisciplinar, así como una idoneidad de equipo técnico e instalaciones que garanticen unos objetivos mínimos ya mencionados. En nuestro Centro todos los pacientes candidatos a $\mathrm{CB}$ deben ser previamente valorados por los Servicio de Endocrinología y Nutrición, Psiquiatría y Anestesiología, y durante al menos un año haber realizado tratamiento médico, sin éxito.

\subsubsection{Objetivos}

La CB es el único tratamiento efectivo a largo plazo de la OM. Tiene como objetivo corregir o controlar la patología asociada a la OM, disminuir la mortalidad, y mejorar la calidad de vida mediante una pérdida de peso suficiente y mantenida (6-8). Se establece como referencia el porcentaje de sobrepeso perdido (PSP) de al menos un 50\% para definir la intervención como un éxito (75) y menos del $25 \%$ un fracaso (76), aunque estos conceptos no están globalmente aceptados. Se considera seguimiento a corto plazo los tres primeros años, el medio plazo entre 3 y 5 años, y largo plazo aquel que va más allá de los cinco años de la intervención (77), momento ideal para valorar sus resultados. Para medir esta pérdida de peso lo podemos realizar también con el porcentaje del exceso de IMC perdido (PEIMCP) $(78,79)$, actualmente el recomendado por revistas de referencia como Obesity Surgery (80). Sin llegar a existir un criterio global de éxito sí que se apunta cada vez más a la inclusión 
de otras variables, como la resolución de comorbilidades, para definir el éxito de la intervención (81).

EI PSP se calcula mediante la fórmula:

$\left(\right.$ Peso inicial - peso actual/peso inicial - peso ideal $\left.{ }^{\star}\right) \times 100$

*según tablas de la MLIC

EI PEIMCP se calcula mediante la fórmula:

$($ IMC inicial - IMC actual/IMC inicial - 25) $\times 100$

El paciente debe entender que se trata de una cirugía funcional que altera la anatomía del aparato digestivo, que puede provocar trastornos nutricionales, por lo que debe realizarse un seguimiento médico a largo plazo. También debe entender que aunque los beneficios físicos son importantes no son el objetivo último de la $\mathrm{CB}$, como tampoco lo es el alcanzar el peso ideal, sobre todo en pacientes con obesidad extrema donde este objetivo es una quimera. Así en un documento de consenso elaborado por la SEEDO y la Sociedad Española de Cirugía de la Obesidad (SECO) en el año 2004 (82) se establecieron las grandes directrices quirúrgicas y se definieron los requisitos que debe tener la técnica bariátrica ideal, actualizados en la declaración de Vitoria avalada por la SECO en el año 2015:

- Segura: morbilidad menor del $7 \%$ y mortalidad menor del $0,5 \%$. Con un rango tolerable de fístulas del 0-4\%.

- Útil para al menos el $75 \%$ de los pacientes, cumpliendo los criterios de éxito: mantener una pérdida del exceso de IMC superior al $50 \%$ e $\mathrm{IMC}<35 \mathrm{Kg} / \mathrm{m}^{2}$.

- Duradera, es decir que el beneficio obtenido persista al menos cinco años o mejor de modo indefinido.

- Reproducible por la mayoría de los cirujanos.

- Pocos efectos secundarios: digestivos y nutricionales. 
- Escasas complicaciones a largo plazo, de tal forma que el índice de reintervenciones al año no supere el $2 \%$.

- Reversible, si no en su anatomía sí desde el punto de vista funcional.

La mortalidad estimada se sitúa por debajo del $0,5 \%(83,84)$ y las complicaciones mayores menores del $5 \%$ (85). Los resultados esperables en cuanto al PSP se resumen en la tabla 2 para las principales intervenciones, así como sus complicaciones.

Tabla 2. Resultados en \% según el tipo de cirugía: Banda gástrica (BG), Gastrectomía vertical (GV) y Bypass gástrico (BPG). Tomados de Dogan et al (66)

\begin{tabular}{|l|c|c|c|}
\hline & BG $\mathbf{( n = 2 4 5 )}$ & $\mathbf{G V}(\mathbf{n = 2 4 5 )}$ & $\mathbf{B P G} \mathbf{( n = 2 4 5 )}$ \\
\hline PSP $(2$ años $)$ & $44.2 \pm 19.8$ & $75.4 \pm 24.7$ & $72.3 \pm 23.6$ \\
\hline Reingresos & - & 4,5 & 0,5 \\
\hline Complicación $<30$ días & 3,7 & 7,6 & 5,7 \\
\hline Fístula & - & 1,6 & 0,4 \\
\hline Reintervención <30 días & 0,8 & 3,7 & 2,9 \\
\hline Complicación >30 días & 28 & 5 & 9 \\
\hline Reintervención >30 días & 26,9 & 10,2 & 4,1 \\
\hline Cirugía de revisión & 20,8 & 9,4 & - \\
\hline Tiempo hasta la revisión (años) & $3.5 \pm 1.2$ & $2.2 \pm 1.3$ & - \\
\hline
\end{tabular}

Esta técnica ideal, que desde luego no existe (86), no exigiría ningún tipo de colaboración por parte del paciente. Sin embargo es sabido que el éxito depende en gran medida de su colaboración: dieta adecuada, ejercicio, toma de suplementos vitamínicos o seguimientos periódicos son condiciones imprescindibles para la cirugía actual (87).

En el caso del BPG se apunta al efecto restrictivo como responsable del $75 \%$ de la pérdida de peso, y el malabsortivo del $25 \%$ (88). 


\subsubsection{Resultados}

Cuando hablamos del BPG hay que introducir conceptos como la calibración del intestino delgado en función del IMC, como consecuencia de los primeros resultados que se obtuvieron con el BPG, donde se evidenció que en IMC altos (>50 kg/m²), conocidos como súper-obesos, los resultados eran peores (89), así en la actualidad se acepta que la longitud del asa alimentaria juega un papel en la pérdida de peso en pacientes súper-obesos, sin influir en la absorción de vitaminas ni minerales (90).

En cuanto a la resolución de comorbilidades, la DM va a tener en torno al $85 \%$ de al menos mejoría y $75 \%$ de resolución $(3,91)$, la HTA un $60 \%$, la DL un $70 \%$ y el SAHS $80 \%$ de resolución (2).

Debemos tener presente que aunque no se encuentren entre los objetivos, vamos a conseguir una disminución de la incidencia de cáncer $(92,93)$, especialmente en mujeres (94), que puede llegar a disminuir en un $60 \%$ la mortalidad por dicha causa (95). Así como una disminución de eventos cardiovasculares (96).

La esperanza de vida en pacientes con obesidad mórbida se reduce entre 5 y 20 años (97), y la cirugía consigue una disminución de la mortalidad respecto a los no operados (8). Si bien en pacientes de edad avanzada y obesidad extrema este descenso podría no existir (98).

\subsubsection{Calidad de vida}

Globalmente la CB debe aspirar a controlar la patología asociada a la OM, y mejorar la calidad de vida del paciente a través de una pérdida de peso suficiente y mantenida en el tiempo.

Para valorar esta calidad de vida hacemos uso del cuestionario BAROS (76) (figura 9), creado en 1998 con el objetivo de valorar resultados en cirugía bariátrica. Consta de una tabla que incluye tres columnas con los principales apartados de análisis: pérdida de peso, mejora de condiciones médicas y calidad de vida (CV). 


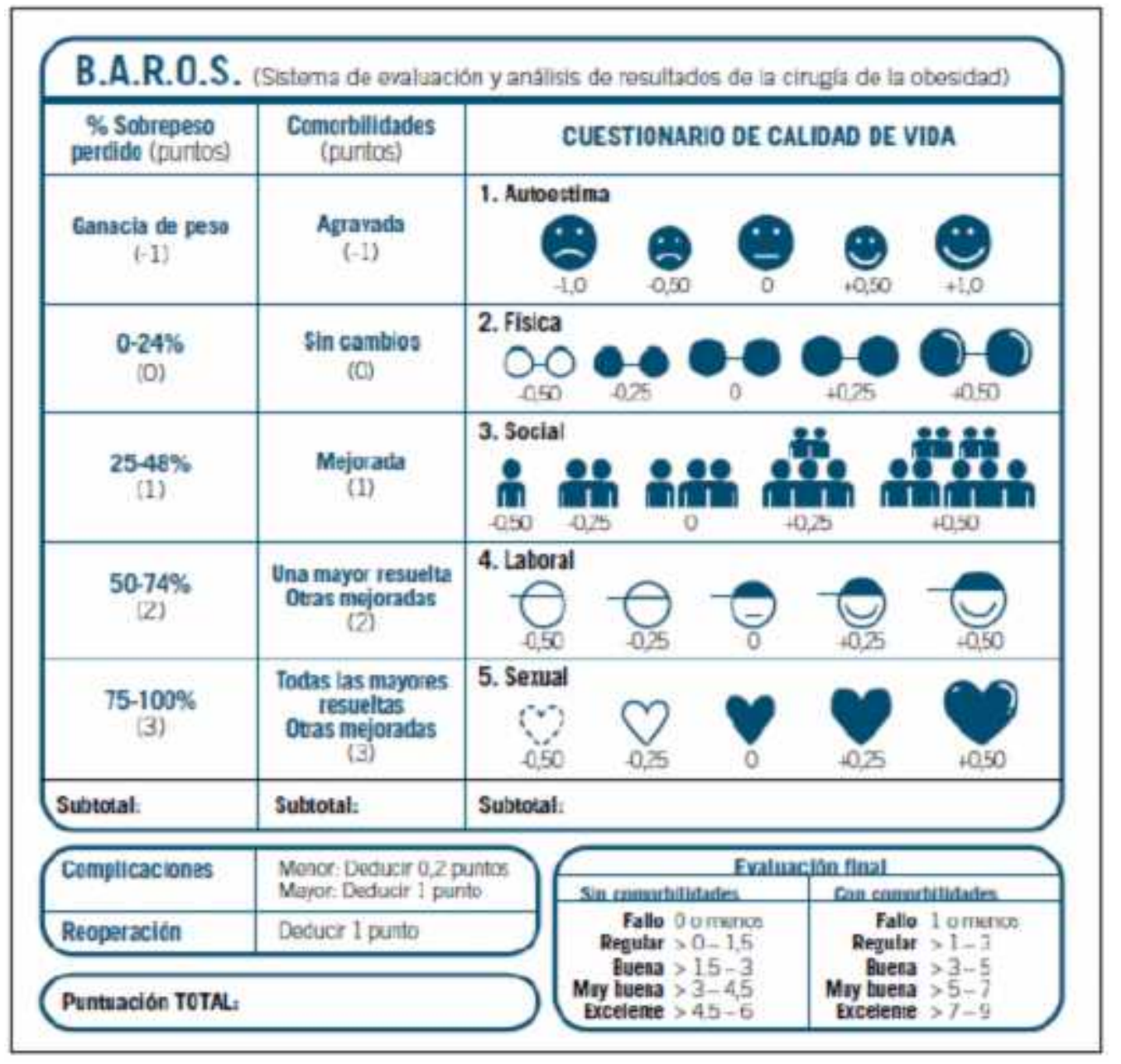

Figura 9. Cuestionario BAROS

El apartado "mejora de condiciones médicas", evalúa las comorbilidades que presenta cada paciente y compara la gravedad de estas pre y post cirugía; para lo cual, se definieron por consenso 7 patologías como comorbilidades mayores (hipertensión arterial, enfermedad cardiovascular, dislipemia, diabetes mellitus tipo II, síndrome de apnea e hipopnea del sueño, osteoartritis e infertilidad); cuyas categorías de respuesta son mejoría (se puede controlar con una disminución de dosis) o resolución (no se requiere medicación para su control). El apartado "CV", corresponde al cuestionario Moorehead-Ardelt que evalúa autoestima, actividad física, vida social, condiciones de trabajo y actividad sexual; usando dibujos simples para ofrecer opciones de respuesta en cada uno de los 5 ítems; de forma tal que en este apartado, se considera prioritario el análisis de la autoestima, razón por la que a diferencia de los otros 4 aspectos evaluados, autoestima tiene un máximo de 1 punto, en cambio las 
otras sólo 0,5 . En cada una de las otras columnas, se otorga una puntuación de -1 a 3 puntos, siendo el negativo reflejo de fracaso. Posteriormente, se suma el número de puntos de cada columna. La presencia de complicaciones mayores o menores o la necesidad de reintervención son consideradas, debiéndose restar a la puntuación total el número de puntos según sea el caso. La puntuación final permite incluir al paciente dentro de 1 de los 5 grupos que definen el concepto de éxito o fracaso. En la eventualidad de realizar evaluación a pacientes sin comorbilidad previa a la cirugía, hay que aplicar otro baremo.

Entre sus limitaciones se encuentra el no existir una referencia preoperatoria en cuanto a la calidad de vida. Además, a pesar de la gran aceptación que ha tenido, el sistema BAROS adolece, en opinión de algunos autores (99), de contener demasiadas valoraciones subjetivas e ignorar otros parámetros, como los relacionados con el ámbito nutricional y gastroenterológico.

\subsubsection{Justificación}

La OM es un proceso crónico, multifactorial y sin tratamiento curativo por el momento. El tratamiento dietético asociado a modificaciones del estilo de vida, ejercicio y terapia conductual, así como el tratamiento complementario con fármacos, consigue pérdidas de peso de alrededor del $10 \%$ a medio plazo, que contribuyen a mejorar algunas de las comorbilidades. Sin embargo debido a los fallos del tratamiento dietético y la eficacia limitada del farmacológico en pacientes con $\mathrm{OM}$, se han buscado alternativas terapéuticas capaces de reducir las comorbilidades graves asociadas, y todo ello dentro de un programa multidisciplinario de especialistas (7): endocrinólogos, cirujanos, nutricionistas, anestesistas, psicólogos y psiquiatras, neumólogos, rehabilitadores,...

El objetivo de la CB es mejorar la salud en el obeso mórbido. Implica reducir la ingesta calórica y/o la absorción de calorías por la alimentación y puede modificar el comportamiento alimenticio promoviendo ingestas pequeñas.

Con estos procedimientos no solo conseguimos una reducción inicial de peso, sino que también se mantiene a largo plazo, siendo su eficacia superior a 
cualquier tratamiento médico, como ha sido confirmado en varios meta-análisis $(2,3)$. La cirugía sin duda plantea un coste elevado, que supone una inversión inicial importante pero según estudios de evaluación económica el gasto que se ahorra a largo plazo, tanto en costes directos como medicamentos, e indirectos como días de baja laboral relacionados con la obesidad (100), consigue un balance final positivo a favor de la CB como medida costo-efectiva.

Sin embargo, hay publicaciones, aunque menos, que no objetivan beneficio económico en los programas de CB $(101,102)$, apuntando la posibilidad de realizar una selección de pacientes con OM y alguna patología asociada que más se puedan beneficiar de la pérdida de peso (como la diabetes o la artrosis), más teniendo en cuenta el momento económico que vivimos $(103,104)$.

Como se puede apreciar tras lo expuesto, el dinamismo y la proyección tanto clínica como investigadora que emana de la CB solo puede entenderse desde una demanda social amplia y creciente, unos resultados no conseguidos hasta la fecha con ninguna otra medida contra la obesidad, un bajo número de complicaciones, y un extenso soporte de la industria farmacéutica.

\subsubsection{Cirugía laparoscópica}

En un estudio económico de la cirugía bariátrica es importante hacer una mención especial a la introducción del abordaje laparoscópico, hoy de uso cotidiano, que como en otras áreas de la cirugía implicó una evolución pero también al menos cambios económicos si no incrementos. Con la laparoscopia conseguimos reducir las complicaciones de pared abdominal como las eventraciones, una complicación frecuente en estos pacientes, y una recuperación más rápida gracias a las bondades ya clásicas como disminución del dolor postoperatorio, íleo, estancia media y de la morbi-mortalidad $(105,106)$. Actualmente es el abordaje de elección, y en su incorporación a la actividad cotidiana encontramos en un registro nacional de EEUU como en el año 2003 el $80 \%$ de las cirugías bariátricas eran abiertas, mientras que en el año 2008 más del 90\% eran laparoscópicas, al tiempo que disminuía la mortalidad (107). 


\subsection{Entendiendo la cirugía metabólica}

El síndrome metabólico (SM) comprende una serie de factores tanto para el desarrollo de la DMT2 como para la aparición de ECV, y para su definición la Federación Internacional de Diabetes (FID) estableció unos criterios diagnósticos de fácil aplicación clínica (tabla 3), que permiten identificar los pacientes con alto riesgo de desarrollar estas patologías.

Tabla 3. Definición de síndrome metabólico por la Federación Internacional de Diabetes

\section{OBESIDAD CENTRAL:}

- $\geq 94 \mathrm{~cm} 0^{\star}$

- $\geq 80 \mathrm{~cm}$ +

- $\quad \mathrm{IMC}>30 \mathrm{~kg} / \mathrm{m}^{2}$ (se asume obesidad central)

Y AL MENOS TRES DE LOS SIGUIENTES FACTORES:

- Triglicéridos: $\geq 150 \mathrm{mg} / \mathrm{dl}$ o tratamiento específico

- Colesterol HDL: $<40 \mathrm{mg} / \mathrm{dl}$ en $0^{\mathrm{t}} \mathrm{y}<50 \mathrm{mg} / \mathrm{dl}$ en + o tratamiento específico

- Tensión arterial: TAS $\geq 130 \mathrm{mmHg}$ o TAD $\geq 85 \mathrm{mmHg}$ o tratamiento

- Glucosa plasmática en ayunas $\geq 100 \mathrm{mg} / \mathrm{dl}$ o diagnóstico de DMT2

Hoy en día todavía se desconoce cuál es el mecanismo por el que el aumento del tamaño de la cintura puede condicionar la aparición de SM. En cualquier caso lo que parece claro es que ese exceso de tejido adiposo visceral es el que desencadena un estado proinflamatorio que conduce a la resistencia a la insulina y a la enfermedad cardiovascular (figura 10).

No está acreditado que la morbimortalidad global del SM sea diferente a la suma individualizada de cada uno de sus integrantes. No obstante, la aplicación de esta clasificación es útil en cuanto permite identificar un grupo de pacientes que tienen un riesgo muy alto de desarrollar DMT2 o ECV. Con este condicionante si bien los tratamientos a aplicar no serán diferentes a los empleados en pacientes sin SM, sí que los objetivos terapéuticos a conseguir van a ser más exigentes y para ello el plan terapéutico ha de ser más agresivo, y esto incluye la obesidad como principal protagonista del SM, lo que nos enlaza con la cirugía, conocida como metabólica. 
La cirugía metabólica $(\mathrm{CM})$ es la consecuencia de unos resultados en pacientes con $\mathrm{CB}$, hasta ahora no conseguidos con ninguna otra medida terapéutica, en la resolución de comorbilidades relacionadas con la obesidad. Además estos resultados sorprenden por no estar directamente relacionados con la pérdida de peso de tal manera que la cirugía cuanto más componente malabsortivo presenta, mejor resuelve patologías como la DM (108), incluso antes de que empiece la pérdida de peso $(109,110)$, así los pacientes pueden ir de alta tras la cirugía con el mismo peso con el que ingresaron, pero sin necesidad de tomar su medicación para la DM. Ante esta evidencia parece lógico pensar que no sólo el IMC debe ser la indicación quirúrgica, incluso podemos bajar este umbral al incluir otras variables como objetivo más allá de la pérdida de peso. Con esta filosofía surge la CM, fruto de las bondades de estas intervenciones.

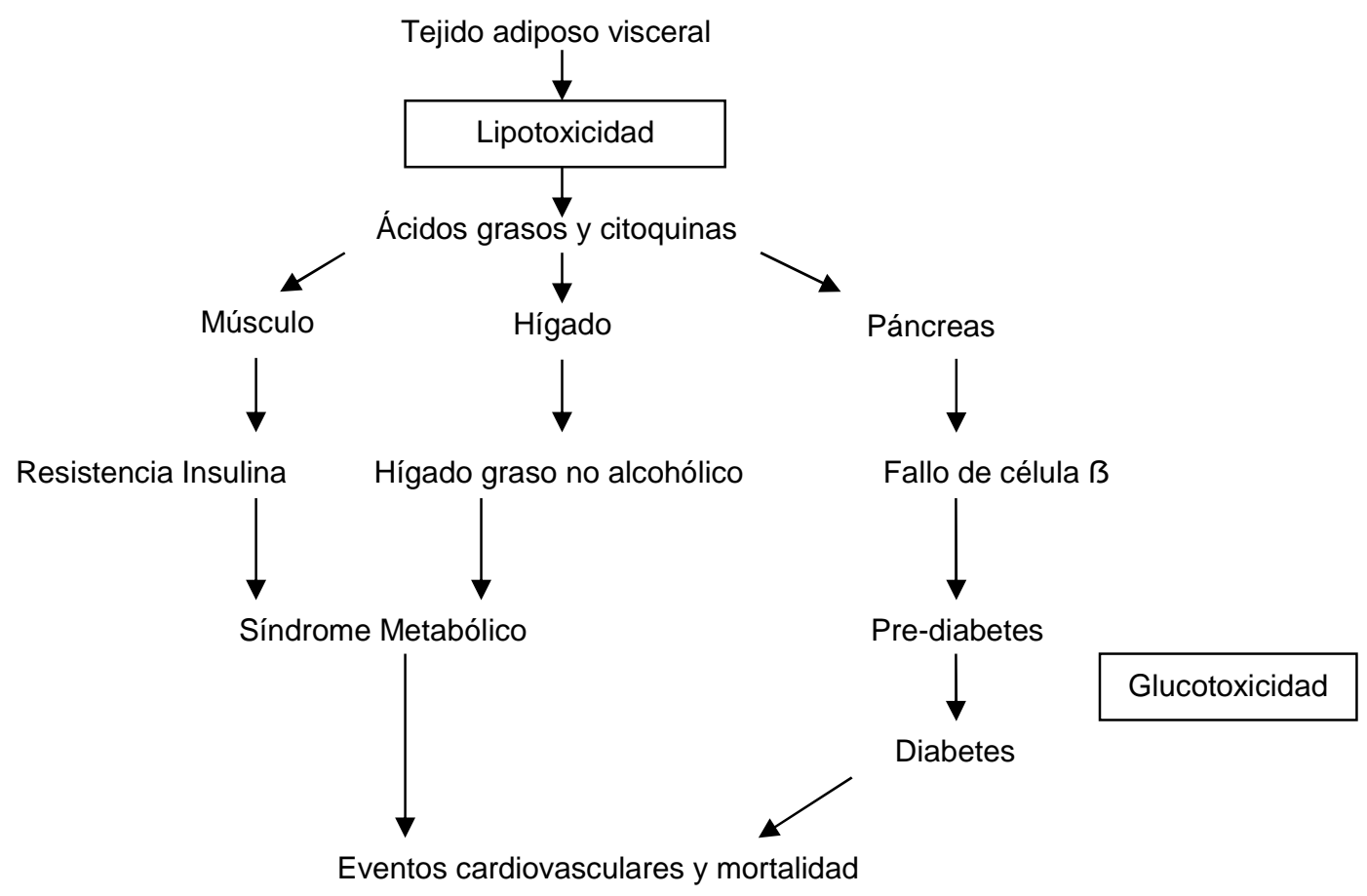

Figura 10. Lipotoxicidad como causa de diabetes mellitus y síndrome metabólico. Tomado de Pok (108) 
Sobre los mecanismos que regulan estos efectos beneficiosos, a raíz de estudios en ratas a las que se realizó un bypass gástrico, Rubino et al (111) postularon la teoría del intestino anterior, según ella sería a este nivel donde se producirían los fenómenos responsables de que mejorase la homeostasis de la glucosa tras el bypass. Frente a esta teoría Melissas et al (112) demostraron otros mecanismos de acción y elaboraron la teoría del intestino posterior, por la cual el paso acelerado de nutrientes a tramos distales del intestino provocaría la liberación de mediadores del tipo de las incretinas GLP-1 y GIP, y el Péptido YY responsables de la mejora tanto de la secreción como de la sensibilidad a la insulina.

De acuerdo con la FID (113), según consenso de 2011, la cirugía debe considerarse una alternativa terapéutica en aquellos pacientes con IMC entre 30 y $35 \mathrm{~kg} / \mathrm{m}^{2}$ con diabetes mal controlada con el tratamiento médico correcto, especialmente en presencia de otras enfermedades de riesgo cardiovascular. En asiáticos, como ya comentamos, podemos bajar 2,5 puntos el IMC.

Ante esta falta de evidencia científica que avale una postura unánime en las indicaciones de CM debido sobre todo a la falta de resultados a largo plazo, las sociedades científicas entre ellas la SECO y la SEEDO aportaron su recomendación, en términos: "consideramos que podrían ser candidatos" (114). 


\subsection{Evaluación económica}

\subsubsection{La empresa hospitalaria}

Ha existido en el pasado un debate sobre si el hospital debía considerarse o no una empresa. Los partidarios de que el hospital no era una empresa señalaban los aspectos éticos de la salud, la enfermedad y la muerte y querían preservar estos temas de la contaminación económica. Los partidarios de la empresa hospitalaria defendían el aserto de que la salud aunque no tenía precio sí tenía un coste, y que había que considerar los aspectos económicos del hospital para permitir su subsistencia. Este debate quedó ya superado hace muchos años y en el ámbito de los sistemas sanitarios públicos, el resultado ha sido considerar al hospital como una empresa, que presenta algunas peculiaridades respecto a una empresa comercial-industrial estándar (115):

- La finalidad del hospital no es lucrativa.

- El producto del hospital es el paciente y no es posible seleccionarlo.

- El hospital es una empresa de servicios y su recurso principal es el personal.

- Están financiados por un presupuesto votado por un ente político.

- Las decisiones fundamentales que se toman en un hospital son clínicas y en la mayoría de los casos están desprovistas de atención económica.

- El trabajo clínico está sujeto a principios éticos y profesionales de la salud, la enfermedad, la vida y la muerte.

\subsubsection{Análisis coste efectividad en la planificación sanitaria}

A partir de los años setenta del siglo pasado, el incremento continuo del gasto sanitario ha motivado una preocupación creciente por parte de los gobiernos. Las razones principales de este crecimiento son el envejecimiento de la población y el cuidado de los enfermos terminales, el aumento de las enfermedades crónicas y de la necesidad de cuidados que generan, la variabilidad en la práctica clínica, las presiones sociales y de los profesionales de la salud en la demanda de servicios, el incremento de la cartera de servicios y la mayor accesibilidad a las prestaciones sanitarias. Además, un aspecto específico que contribuye de manera importante al gasto sanitario creciente es 
la introducción de nuevas tecnologías, tratamientos médicos y quirúrgicos cada vez más sofisticados y en pacientes hasta la fecha no subsidiarios de los mismos.

Las mejoras de salud de la población, expresadas tanto en términos cuantitativos (esperanza de vida y mortalidad infantil) como cualitativos (esperanza de vida libre de incapacidad), han continuado produciéndose a un ritmo que se relaciona no sólo con la mejora progresiva de los niveles de vida de la población, sino también con las cuantiosas inversiones producidas a lo largo de estos años en el sector sanitario. Esta evolución se enmarca en un contexto histórico de mediados de los ochenta, por el que la Ley 14/1986 General de Sanidad (LGS) define el Sistema Nacional de Salud (SNS) español, por la universalidad del derecho de asistencia así como por una financiación y provisión mayoritariamente públicas. A su vez confiere competencias a las Comunidades Autónomas en materia de sanidad, que paulatinamente asumen hasta estar finalmente recogidas en la Ley 16/2003 de Cohesión y Calidad del SNS. Las principales consecuencias surgidas de la LGS se pueden resumir en los siguientes puntos (116):

- Se amplía la cobertura hasta alcanzar la casi totalidad de la población.

- Evoluciona la forma de financiar el sistema, que pasa desde 1986 hasta 1997 a depender de las aportaciones del estado (tabla 4). En la actualidad (última referencia 2011) la financiación depende en su mayor parte de las administraciones regionales.

Tabla 4. Gasto sanitario según el agente de financiación (en \%). Fuente MSSSI

\begin{tabular}{|l|l|l|l|l|l|}
\hline & 1986 & 1990 & 1994 & 1997 & 2011 \\
\hline Aportaciones del Estado & 23,77 & 68,77 & 70,23 & 91,85 & 0,07 \\
\hline Cotizaciones sociales & 74,29 & 27,21 & 27,12 & 5,98 & 6,14 \\
\hline Serv. prestados a terceros & 0 & 2,67 & 1,65 & 1,93 & \\
\hline
\end{tabular}




\subsubsection{Normas de decisión para el análisis coste-efectividad}

Hasta la fecha, el método más utilizado para el análisis económico ha sido el análisis coste-efectividad (ACE), en el que los beneficios de las intervenciones sanitarias se miden en unidades naturales. Aunque esta forma de análisis tiene una gran consistencia cuando una intervención sanitaria domina a una intervención alternativa (es menos costosa y tiene una mayor efectividad), estos resultados son difíciles de interpretar cuando la intervención tiene una mayor efectividad pero también un mayor coste, y debemos ser cautos.

Para poder juzgar que el beneficio en salud tiene un coste adicional, los economistas utilizan la noción del coste de oportunidad, que se define como el valor de los recursos en la mejor alternativa disponible.

EI ACE permite la comparación de programas cuyos resultados pueden medirse en las mismas unidades. En el momento en el que asignamos un valor monetario a esas unidades como denominador común, estamos ante un análisis coste-beneficio. Cuando un programa o intervención sanitaria ocasiona mayores costes y también mejores resultados, el análisis coste-efectividad no proporciona información suficiente para decidir si éste debe ser aplicado o financiado. Con el ACE, en este caso, se requiere algún criterio externo sobre el coste máximo por unidad de efectividad que estamos dispuestos a financiar. Es decir, aunque el ACE en apariencia evita la valoración monetaria de los efectos sobre el estado de la salud, para que sus resultados sean utilizados en la toma de decisiones se requiere que "alguien" establezca de forma implícita o explícita ese valor monetario para el efecto sobre la salud (117).

La medida de efectividad (de beneficio sanitario) que necesitamos para realizar un estudio coste-efectividad dependerá del objetivo del programa o tratamiento a evaluar.

Los hospitales necesitan normas de comportamiento al consumir los recursos que deben proyectarse a los servicios, que serán los que determinarán la buena praxis ante una determinada patología para que suponga el mejor beneficio al paciente y requiera un menor consumo de recursos. 
Otro aspecto relacionado con la medida de la efectividad está relacionado con el objetivo de cualquier evaluación económica. El objetivo de la evaluación económica es asignar recursos siguiendo las preferencias de la población. Esto hace que sea relevante la distinción entre dos tipos de medidas de resultados, los resultados intermedios y los finales. Si queremos asignar los recursos para producir bienes valorados por la población, necesitamos información sobre resultados finales.

El ACE es una técnica de evaluación económica que trata de comparar el coste y el efecto clínico de dos o más intervenciones alternativas, potencialmente competidoras y, en general, mutuamente excluyentes, para determinar cuál de ellas es mejor desde un punto de vista económico y clínico. La técnica de evaluación económica elegida depende de la naturaleza de los beneficios que se van a estudiar. En el caso del ACE los beneficios se expresan en términos no monetarios relacionados con los efectos de las alternativas terapéuticas en la salud, y pueden ser expresados como el porcentaje de curación, el porcentaje de pacientes que alcanza un determinado objetivo terapéutico, etc., mientras que en un ACU (análisis coste-utilidad) o en un ACB (análisis costebeneficio) dichos beneficios se expresan respectivamente, en años de vida ajustados por calidad (AVAC) u otras utilidades y beneficios monetarios.

\subsubsection{Coste-efectividad y cirugía bariátrica}

En 1995 la FDA (Food and Drug Administration) emitió un informe en el que se apuntaba que la cirugía costaba la mitad que el tratamiento médico por cada libra de peso perdida (118). Eran los primeros estudios coste-efectividad del BPG, y desde entonces con la mejora en disminución de costes directos y seguridad de la cirugía, se han ido repitiendo los estudios que muestran la cirugía como un tratamiento coste-efectivo.

Si bien es difícil contestar a la pregunta de si la cirugía bariátrica es costeefectiva, y probablemente a día de hoy no tenga una respuesta global, los estudios de los que disponemos nos orientan a que en términos económicos es adecuado o justificado operar la obesidad, siempre teniendo en cuenta que estamos haciendo prevención terciaria, o sea que el daño ya existe y nos 
limitamos a "paliar" o minimizar sus complicaciones, que es la acción más cara y de peores resultados. Una vez asumido esto debemos tener en cuenta que los estudios en este sentido son mayoritariamente de EEUU, un sistema sanitario radicalmente distinto al nuestro, con unos costes muy superiores y basado en la medicina privada, lo que lo aleja de la sanidad y la salud como derecho. La variabilidad en Europa también es amplia (119), tanto en financiación como en número de intervenciones por millón de habitante (figura 11). Si nos aproximamos a sistemas sanitarios mayoritariamente públicos para valorar estudios económicos, como el del Reino Unido conocido como National Health Service (NHS) y basado en las guías del National Institute for Health and Care Excellence (NICE) de obligado cumplimiento a nivel nacional, consideran coste-efectiva la cirugía bariátrica, calculando el punto de corte para que así sea en las $30.000 £$, mientras que la ratio CE para el BPG es de $6.289 £$ (120). Desde nuestro entorno es envidiable un sistema nacional de salud cohesionado y que basa sus políticas sanitarias en resultados médicos y económicos, en continua revisión y actualización. Hay que señalar que la cirugía bariátrica resulta barata en nuestro entorno (7.500€ aproximadamente), como indica en el estudio de Sánchez-Ramos (9), de los pocos trabajos que hay en este sentido en nuestro país; el problema es que está basado en los GRDs (grupos relacionados por el diagnóstico) como método de valoración económica, el más utilizado en nuestro país y el que manejan nuestros gestores, que está lejos de una correcta valoración del coste real (121) entre otros motivos por no tener en cuenta los días de ingreso; y agravado por el hecho de no tener políticas sanitarias autonómicas comunes ni dar una respuesta adecuada a la demanda social existente. Con todo esto quizá no deberíamos preguntarnos si esta cirugía es coste-efectiva, sino hasta qué punto debemos esforzarnos en demostrarlo para justificar su aplicación universal y en plazos similares a otras patologías. Recientemente desde la SEEDO y SECO se denunció en rueda de prensa (122) la situación de demora quirúrgica, al estar la cirugía bariátrica fuera de las listas de espera convencionales. 


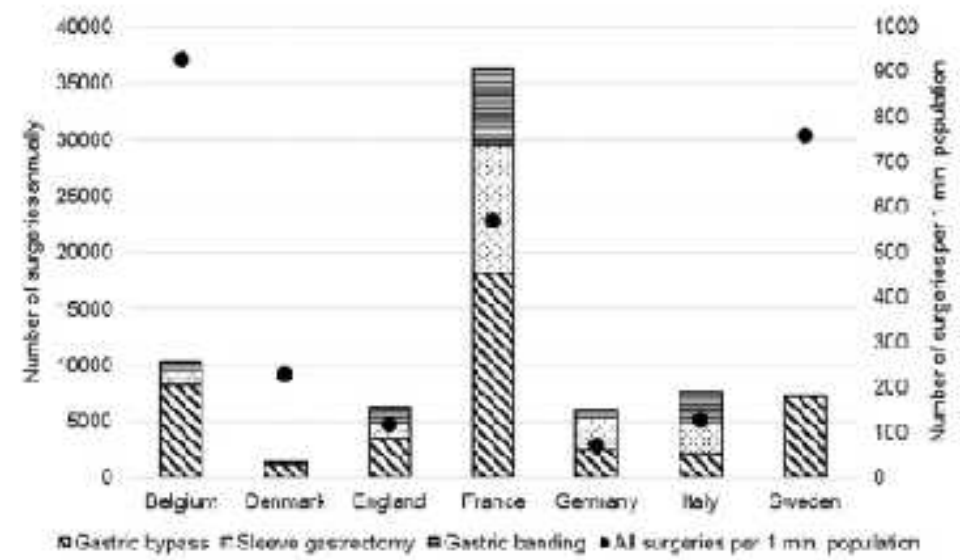

Figura 11. Número de cirugías bariátricas en Europa. Tomado de Borisenko et al (119)

La superioridad del tratamiento quirúrgico de la OM frente al médico es clara, mientras que el ser CE aún permanece a debate. La cirugía plantea un coste elevado, que supone una inversión inicial importante, y de la que algunos autores dudan que se consiga recuperar a lo largo de la vida (60). Según la mayoría de los estudios de evaluación económica el gasto que se ahorra a largo plazo tanto en costes directos como en medicamentos, e indirectos como días de baja laboral relacionados con la obesidad, consigue un balance final positivo a favor de la cirugía como medida costo-efectiva (123-125), con ciertos estudios que concluyen que se recuperan los gastos quirúrgicos entre los dos y los cuatro años $(126,127)$, sobre todo en pacientes con patología asociada como DM $(128,129)$. Según un estudio de Borisenko et al $(130)$ el BPG sería la opción que más beneficio económico tiene y su sustitución por la GV o BG en un escenario hipotético supondría pérdida tanto de AVAC como económica.

Sin embargo hay publicaciones, menos, que no objetivan beneficio económico en los programas de cirugía bariátrica $(101,102)$, y apuntan a la selección de pacientes con obesidad mórbida y alguna patología asociada que más se puedan beneficiar de la pérdida de peso (como la diabetes o la artrosis), más teniendo en cuenta el momento económico que vivimos (103).

Generalmente cuando se habla de CE en OM se comparan distintos tipos de cirugía con el tratamiento médico convencional. La mayoría de los estudios concluyen que la cirugía es más costosa pero es mejor en términos de pérdida de peso, mejoría de comorbilidades, longevidad y calidad de vida. Solo en un 
estudio del Reino Unido en pacientes con DM, a los cinco años la cirugía fue más cara (131).

Si nos centramos en la disminución de fármacos como argumento que justifique esta intervención las referencias también son variadas. Desde aquellos que señalan que el gasto generado en la lista de espera, para un periodo medio de 22 meses, sobrepasa el coste de la cirugía (132) hasta los que encuentran un aumento del coste en fármacos a los seis años de la cirugía (133). Lo más habitual es encontrar una reducción del gasto en fármacos para la mayoría de las comorbilidades mientras que en otras de resolución más compleja como la depresión, no siempre se produce (134). La reducción global es de unos dos fármacos por paciente (135). Otros autores señalan los siete años para que el ahorro en fármacos supere al valor de la cirugía (136). Y si bien la medicación preventiva es más intensa en los primeros momentos tras la intervención posteriormente disminuye, y se reduce a vitaminas y minerales generalmente, representando un gasto pequeño. Con esto, y sabiendo lo difícil que es generalizar en esta patología, intuimos que el BPG tiene beneficio económico por disminución de fármacos y dispositivos médicos, pero no queda claro si es coste ahorro.

Parece adecuado adoptar medidas encaminadas a disminuir el tiempo de lista de espera quirúrgica (LEQ) y facilitar el acceso a la $C B$ en pacientes que no responden al tratamiento médico (137-139), un tema controvertido ya que la cobertura de la CB es muy heterogénea geográfica, económica y políticamente.

\subsubsection{Evaluación del gasto hospitalario}

El coste del proceso de hospitalización podemos realizarlo mediante dos modelos bien diferentes; el tradicional (top-down) y el de imputación de coste por paciente y episodio (bottom-up). Veremos cómo hemos evolucionado desde el cálculo estimado de coste por proceso, en función del peso relativo de cada GRD (modelo top-down), al coste del proceso a través de la asignación de consumos y productos al episodio de hospitalización de cada paciente y la posterior reconstrucción del coste del proceso como suma de los costes de los pacientes atendidos por ese proceso (modelo bottom-up) (140). 


\subsubsection{Los sistemas de clasificación de pacientes (SCP)}

En los años ochenta, las entidades aseguradoras norteamericanas comenzaron a utilizar nuevos métodos, que se agrupaban bajo los SCP, para contratar y pagar a los hospitales (141). El sistema quedó definitivamente implantado a nivel nacional el 1 de Octubre de 1983 (142).

Estos sistemas se nutren de la información disponible en la historia clínica de cada paciente dado de alta que una vez codificada, permite agrupar las altas del hospital en grupos homogéneos. Los grupos se confeccionan sumando las altas. De ahí que se les denomine grupos de iso-consumo e iso-diagnóstico. La configuración de los grupos se efectuó:

- A partir de la observación empírica de millones de historias clínicas, analizando el consumo de recursos de cada alta hospitalaria y la similitud de su diagnóstico; por este método se creó el sistema de clasificación de pacientes DRG (Diagnosis Related Group), conocidos en nuestro entorno como los Grupos Relacionados por Diagnóstico (GRD).

- A partir de protocolos teóricos en los que se señalan los cuidados que requiere cada posible proceso a atender en un hospital y la evaluación también teórica, de su coste; éste fue el método utilizado para la creación del sistema de clasificación de pacientes PMC (Patient Management Categories).

Esta clasificación se refiere a la producción final del hospital (las altas hospitalarias una vez atendidas), y no a productos intermedios (estancias, intervenciones quirúrgicas,....).

Estos sistemas aunque representan un avance significativo para el control de la gestión de los hospitales, no identifican la totalidad de las actuaciones que se llevan a cabo en un hospital ya que únicamente sirven para los procesos que requieran hospitalización, quedan por tanto fuera de control aquellos servicios a pacientes no ingresados como las consultas médicas, la cirugía ambulatoria o las urgencias sin ingreso y otros tratamientos de carácter ambulatorio. Además hay otras limitaciones como el agrupar pacientes por una determinada 
patología sin discriminar valores tan importantes como la estancia o depender de una correcta codificación realizada por personal no sanitario.

La introducción de los SCP en los hospitales españoles supuso una auténtica innovación en los métodos de gestión. Los principales cambios fueron:

- Variación de los mecanismos de financiación, al pasar de presupuestos discrecionales basados en la estructura del hospital, a la financiación vinculada a la prestación de servicios.

- Potenciación de los sistemas de información del hospital, tanto en su vertiente productiva identificando los procesos de forma individualizada, como en su vertiente económica, al desarrollar una contabilidad analítica que permita identificar los costes que implica cada servicio que se presta, y poder compararse con hospitales de características similares.

- Reorientación de la gestión, que anteriormente se centraba en la estructura del hospital, hacia el cliente-paciente, lo que conllevará importantes cambios culturales y organizativos.

- El desarrollo de políticas de calidad relacionadas con los procesos atendidos y especialmente la disponibilidad de indicadores de resultado para determinados procesos.

\subsubsection{Grupos relacionados por el diagnóstico}

Son el método tradicional utilizado en contabilidad analítica, basado en su peso relativo. En este sistema los costes generados en el periodo de cálculo, una vez situados en los Grupos Funcionales Homogéneos (GFH) finales de cada una de las líneas de actividad (consultas, actividad ambulante, hospitalización, etc.), se trasladan hasta los productos finales en función del peso o valor relativo de cada producto. En la línea de hospitalización, el coste de cada GFH final se imputa sobre los productos finales o GRD generados de forma proporcional al peso relativo de cada uno. El proceso de cálculo es sencillo, primero se calcula el coste de la Unidad de Complejidad Hospitalaria (UCH) de cada GFH final (coste total del GFH entre peso total de los GRD generados en 
el periodo) y posteriormente se calcula el coste unitario de cada GRD multiplicando el coste unitario de la UCH por el peso relativo de cada GRD.

Este sistema de cálculo de costes es el Full Costing o Costes Completos, de tal forma que el coste de un proceso estará compuesto de sus costes directos, obtenidos en función de los consumos de recursos generados por la atención sanitaria, costes repercutidos de otros servicios y costes estructurales del propio servicio que atiende al paciente.

\section{Elaboración}

El coste por proceso es el resultado de obtener el coste medio de todos los pacientes que han sido dados de alta en un determinado GRD. El coste por proceso a partir de los GRD se basa en las siguientes características:

- Se obtiene a partir de las altas codificadas.

- Se descuenta el coste generado por los pacientes dados de alta sin codificación de su proceso y de los pacientes en curso (pacientes ingresados).

El sistema de coste por GRD, basado en la asignación de recursos al episodio clínico, nos proporciona información en los siguientes niveles:

Nivel paciente: Detalla el coste de los recursos generados en cada uno de los pacientes que han sido dados de alta, en los distintos GRD.

Nivel servicio: Detalla el coste medio de cada uno de los GRD, en los distintos servicios del hospital, así como su detalle de costes.

Nivel hospital: Detalla el coste medio global de cada uno de los GRD en el centro hospitalario.

\section{Ventajas de los GRD}

- Uso extendido tanto en EEUU como en Europa.

- Modificaciones periódicas.

- Fáciles de implementar. 
- Constituyen un lenguaje fácil de comprender por los médicos.

- Representan un producto hospitalario homogéneo en el cual es posible estudiar potenciales problemas de calidad: tasas de mortalidad por GRD, infecciones nosocomiales por GRD, reingresos por GRD, estancias inadecuadas, etc...

Desde el año 1992, los hospitales del antiguo INSALUD cuentan con dos herramientas básicas de gestión, la codificación del Conjunto Mínimo Básico de Datos (CMBD) de las altas hospitalarias y la contabilidad analítica por servicio, desarrollada en general dentro del Proyecto Signo de Gestión Analítica por Centros de Coste o por Grupos Funcionales Homogéneos (GFH) (143).

Los GRD con los que vamos a trabajar son quirúrgicos:

\section{- 288. Procedimientos quirúrgicos para obesidad.}

Es un GRD quirúrgico que agrupa a pacientes ingresados por obesidad a los que se les ha practicado una intervención como: gastroenterostomía, BPG, mamoplastia reductora $u$ otras plastias reductoras.

No existe este GRD con complicación.

- 565. Procedimientos endocrinos, nutricionales y metabólicos excepto amputación miembro inferior con complicación mayor.

Es un GRD quirúrgico que agrupa a pacientes ingresados por una enfermedad endocrina, nutricional o metabólica a los que se les ha practicado cualquier intervención excepto amputación de miembro inferior y que además, tienen otro diagnóstico etiquetado de complicación o comorbilidad mayor como: hematoma, hemorragia, dehiscencia de herida 0 seroma postoperatorios, insuficiencia renal aguda o ulcus con hemorragia aguda. Las intervenciones que con más frecuencia se realizan a estos pacientes son cirugía tiroidea o suprarrenal.

- 75. Procedimientos torácicos mayores.

- 154. Procedimientos sobre estómago, esófago y duodeno, edad mayor de 17 años, con complicación. 
- 877. Oxigenación extracorpórea de membrana o traqueostomía con ventilación mecánica más de 95 horas con procedimiento quirúrgico mayor o traqueostomía con un diagnóstico motivo del ingreso que no es de boca, laringe o faringe con procedimiento quirúrgico mayor.

Es el GRD quirúrgico de mayor peso que existe.

Aparte de los mencionados, existe un GRD médico, que registrará el ingreso necesario para la colocación de un BIG en los pacientes que lo precisen. Es el GRD 297. Trastornos nutricionales y metabólicos misceláneos en personas mayores de 17 años, sin complicación.

Debido a la necesidad de tener un mayor número de GRD que se ajusten a la realidad que representa la CB en este momento, tanto por diversidad como por volumen, desde octubre de 2011 en EEUU existen tres nuevos GRD para mejor definición de estos procedimientos y otros seis que representan los casos complejos de complicación mayor $(144,145)$, que aún no están aplicados en nuestro país. Se estima que a lo largo del 2016 comenzarán a utilizarse los GRD refinados (GRDR), que contemplan toda la actividad desarrollada a nivel hospitalario tanto la hospitalización como la actividad ambulatoria, es decir, contempla los procesos de hospitalización, los procedimientos ambulatorios como la cirugía mayor y menor ambulatoria, las urgencias no hospitalizadas, las consultas externas por especialidades y las pruebas complementarias como laboratorio y radiología, etc. Lo que supone una gran evolución con respecto a los actuales.

Pese a esto, los hospitales deben de ir avanzando en el desarrollo de su gestión clínico-financiera y por lo tanto, deben sustituir este sistema estimado de cálculo por GRD por un sistema basado en la asignación de recursos al paciente, más preciso y más ajustado al momento en el que nos encontramos.

\subsubsection{Asignación de costes directos al paciente}

La asignación por paciente y episodio clínico permite construir el episodio clínico desde el punto de vista de consumo de prestaciones, en nuestro modelo se construye por agregación de tres grandes conceptos: 
- Coste directamente imputado a paciente y episodio (implantes, unidosis, dietas, programas de trasplantes, etc.).

- Coste de los productos y actividades del catálogo, consumidos en el episodio clínico (analíticas, técnicas diagnósticas y terapéuticas, tiempos de quirófano, de reanimación, estancias, etc.).

- Resto de costes no imputables directamente a paciente y episodio de hospitalización, que se traslada hasta el episodio clínico mediante el criterio "número de estancias a cargo del GFH de hospitalización".

La bondad de este sistema es directamente proporcional a la magnitud acumulada en los dos primeros conceptos. Hay que indicar que esta forma de trabajar supone la introducción en el programa informático de un número de registros muy superior al que sería si se trabajase con el método tradicional.

\subsection{Gasto y contexto sanitario}

Siempre que se habla del futuro de la sanidad pública en España se cita al envejecimiento de la población como el principal desafío que deberán afrontar las autoridades. Pero lo cierto es, que tan importante como la evolución demográfica, es el problema de la obesidad.

El gasto sanitario público en el año 2013 en España supuso 61.710 millones de euros, lo que representa el 5,9 por ciento del producto interior bruto (PIB). El gasto per cápita ascendió a 1.309 euros por habitante (tabla 5) (146).

Tabla 5. Gasto sanitario público en España y Asturias

\begin{tabular}{|l|c|c|c|c|c|}
\hline & $\mathbf{2 0 1 0}$ & $\mathbf{2 0 1 1}$ & $\mathbf{2 0 1 2}$ & $\mathbf{2 0 1 3}$ & $\begin{array}{c}\text { Principado de } \\
\text { Asturias } \\
\mathbf{( 2 0 1 3 )}\end{array}$ \\
\hline Millones de euros & 69.417 & 68.050 & 64.153 & 61.710 & 1.520 \\
\hline Porcentaje sobre PIB & $6,40 \%$ & $6,30 \%$ & $6,10 \%$ & $5,90 \%$ & $7,10 \%$ \\
\hline Euros por habitante & 1.475 & 1.442 & 1.357 & 1.309 & 1.423 \\
\hline
\end{tabular}


Se estima que los pacientes obesos consumen entre el 7 y el $10 \%$ del gasto sanitario total (9). Profundizando en el problema socio-sanitario que supone la obesidad encontramos un estudio patrocinado por la empresa ROCHE y publicado en 1999 (147), que hace una estimación del gasto que se genera con la obesidad, y que a día de hoy sigue siendo referencia de muchos artículos para asumir esta cifra del 7\%. Se trata del Estudio Prospectivo Delphi: "Costes sociales y económicos de la obesidad y sus patologías asociadas (hipertensión, hiperlipemias y diabetes)". En este informe se han valorado tanto los costes directos como los indirectos:

1. En los costes directos se incluyen los derivados del gasto en consultas y fármacos para el tratamiento de la obesidad, y también parte del gasto de patologías originadas por la obesidad, como son la DMT2 y las enfermedades musculoesqueléticas o cardiovasculares.

2. Los costes indirectos se asocian a la pérdida de productividad derivada de las propias enfermedades y de la discapacidad que generan, así como las muertes prematuras causadas por la obesidad y una fracción de la originada por las patologías asociadas.

Por último, se ha incluido un capítulo muy importante y no siempre valorado que es el del gasto particular; en él se incluye toda la partida económica que los obesos, de forma personal, gastan en tratar de resolver su problema ( $p$. ej.: herbolarios, masajes, fajas adelgazantes, aparatos de gimnasia, etc.). El estudio nos revela una realidad no suficientemente ponderada: nada menos que el $80 \%$ de los obesos de nuestro país con independencia de su nivel económico o social, realizan un gasto particular en tratar de combatir su obesidad que alcanzaría un promedio de $60 €$ mensuales. Es decir, que la inmensa mayoría de los obesos españoles consumen una parte nada despreciable de sus recursos económicos en tratar de adelgazar y por añadidura mediante remedios como los descritos previamente de dudosa 0 nula eficacia. También debemos señalar que el $65 \%$ de este cálculo estimado entra dentro del apartado de gasto particular, lo que debe al menos poner en duda o cuestionarnos la fiabilidad de estos resultados. 
Estos datos ponen de manifiesto el enorme impacto económico que tiene la obesidad tanto a nivel de la política nacional global como de la contabilidad doméstica, aparte de sus consideraciones de salud pública. Sin duda este es un poderoso argumento que debe incitarnos a plantear medidas para controlar este problema.

En este entorno deben primar acciones coste-efectivas, y determinadas prestaciones sanitarias como la cirugía bariátrica, que socialmente están cuestionadas por la creencia de que el paciente obeso es responsable de su enfermedad, están obviamente en el punto de mira de los gestores.

La prevención puede mejorar la salud a un coste más bajo comparada con muchos tratamientos ofrecidos hoy en día por nuestro sistema de salud, pero el principal problema es que a corto plazo (148) no se muestran tan costeefectivos como al largo (149), y nuestros responsables políticos no se caracterizan por tomar medidas con resultados a largo plazo.

En lo que respecta al Principado de Asturias, su presupuesto sanitario se ha reducido en más de 150 millones de euros en los últimos años (1.639.961.000 del año 2010 a los 1.485 .056 .000 del año 2014), a lo que se suma la apertura de dos nuevos hospitales a lo largo del 2014.

A nivel nacional debemos recordar que en el año 2008 entramos en recesión, en el contexto de una crisis económica mundial que afecta principalmente a los países desarrollados, y si bien esta situación de recesión finalizó el tercer trimestre del año 2013 (150), se puede decir que España aún sigue en crisis. 



\section{HIPÓTESIS}

La cirugía bariátrica supone el único tratamiento efectivo de la obesidad mórbida, tanto en la reducción inicial del peso como en su mantenimiento a largo plazo. Sin embargo el procedimiento (preparación y estudios preoperatorios, gastos de quirófano, o estancia postoperatoria entre otros) requiere una importante inversión inicial, hasta el punto de no estar incluido en la cartera de servicios de muchos países o compañías aseguradoras. Pero mientras se arbitran medidas profilácticas, la obesidad mórbida existe y sus comorbilidades asociadas requieren un gasto sanitario ingente. Si con la cirugía disminuyesen los costes directos, indirectos e intangibles generados por la enfermedad, hasta compensar los relacionados con el acto quirúrgico, tendríamos unos resultados favorables en términos de coste-efectividad, perfectamente asumibles por la comunidad e incluso podrían extenderse las indicaciones a la obesidad no mórbida con comorbilidades mayores, en un momento en el que la cirugía metabólica ha abierto grandes esperanzas para el control de las enfermedades endocrino metabólicas ligadas a la obesidad. 

OBJETIVOS 



\section{OBJETIVOS}

a. Principal:

Evaluación económica de un programa de cirugía bariátrica.

b. Secundarios:

Resultados a corto plazo de la cirugía bariátrica, en pérdida de peso, comorbilidades, calidad de vida y modificación de la medicación.

Estimación de gastos generados, mediante coste por paciente y coste por proceso, y comparación de los mismos.

Identificar los pacientes que más se benefician de este tratamiento.

Comparación del abordaje quirúrgico abierto con el laparoscópico en términos de coste- efectividad. 



\section{PACIENTES Y METODOLOGÍA}





\section{PACIENTES Y METODOLOGÍA}

\subsection{Universo de estudio}

Hospital Universitario Central de Asturias (HUCA), hospital de tercer nivel situado en Oviedo, Centro de referencia del Servicio de Salud del Principado de Asturias (SESPA) y hospital del Área Sanitaria IV de Asturias; es el único Servicio de Cirugía General del Principado donde se realiza cirugía bariátrica. El nuevo HUCA se inauguró en el mes de junio de 2014, en diferente ubicación y con menor número de camas. En el momento del estudio el HUCA contaba con: Hospital Covadonga, Instituto Nacional de Silicosis, Hospital MaternoInfantil y el Hospital General de Asturias. Disponía de 1.355 camas y 25 quirófanos. Da cobertura a una población cercana a los 350.000 habitantes, de ellos aproximadamente 300.000 son adultos. En el caso de la cirugía bariátrica cubre toda la población asturiana, es decir 1.051.229 personas en el año 2015 (151).

\subsection{Declaración ética}

El Comité de Ética de la Investigación del Principado de Asturias autorizó este Proyecto de Investigación ( $n^{\circ}$ 10/16), considerando que reúne las condiciones éticas para poder realizarse.

\subsection{Diseño del estudio}

\subsubsection{Pacientes incluidos}

El periodo de tiempo de estudio comprende desde octubre del año 2003 momento en el que se inició el programa de cirugía bariátrica en el HUCA y diciembre de 2012, fecha elegida para contar con el margen de seguimiento. Se han seleccionado los pacientes a los que se les realizó cirugía primaria de bypass gástrico, todos ellos se ajustaban a los criterios establecidos por el 
equipo multidisciplinar de cirugía bariátrica, así como todos los estudios preoperatorios se realizaron según protocolo. Eliminamos los 30 primeros pacientes de cirugía abierta y los 30 primeros de cirugía laparoscópica, considerados como curva de aprendizaje, y nos queda un total de 307 pacientes, objeto de este trabajo.

\subsubsection{Tipo de estudio}

Sobre los pacientes sometidos a BPG primario se realizó un estudio que incluye dos aspectos: uno clínico que queda registrado en un protocolo de recogida de datos (figura 12) y otro económico de coste del procedimiento.

En los pacientes con IMC mayor de $60 \mathrm{~kg} / \mathrm{m}^{2}$, o mayor de $50 \mathrm{~kg} / \mathrm{m}^{2}$ y con comorbilidades importantes, se indicó la colocación de balón intragástrico (BIG) previo a la cirugía. Los pacientes con BIG siguieron un protocolo adicional y complementario (figura 13). Ingresaron el día previo a su colocación y un endoscopista experto lo efectuó en un quirófano de Cirugía General, bajo sedación por un anestesista. El protocolo se completó con posteriores seguimientos mensuales hasta su retirada de manera ambulatoria en la sala de endoscopias, cuatro meses después. El BPG se realizó un mes más tarde para minimizar complicaciones.

Tras la intervención, al momento del alta, se completó un formulario de recogida de datos derivados del ingreso de la cirugía como viene recogido en la figura 14 y se introdujeron en la base de datos.

Para la recogida de datos más detallados para nuestro estudio hicimos un protocolo orientado a la evolución ponderal y modificación de la medicación (figura 15). 
Nombre y Apellidos

№ de Historia

Fecha Nacimiento

Edad

Dirección

Teléfono

ANTECEDENTES PERSONALES:

Alergias medicamentosas

Tabaco

Alcohol

Grado de actividad física

Situación laboral

Enfermedades previas:

Intervenciones quirúrgicas:

\section{PRESENCIA DE COMORBILIDADES:}

- HTA

- DM

- Varices

- ERGE

- Cardiopatía

- Apnea del sueño

- Colelitiasis

- Amenorrea

- Dislipemia

- Artrosis

- Depresión

- HT intracraneal

- Incontinencia urinaria

Tratamiento medicamentoso actual:

\section{EXPLORACIÓN FÍSICA:}

Tipo: Ginoide Androide

$\begin{array}{llll}\text { Talla }(\mathrm{m}): & \text { Peso }(\mathrm{kg}): & \mathrm{IMC}\left(\mathrm{kg} / \mathrm{m}^{2}\right): & \text { Cuello }(\mathrm{cm}):\end{array}$

Perímetro cintura $(\mathrm{cm}): \quad \quad$ Perímetro cadera $(\mathrm{cm}): \quad$ Índice cintura/cadera:

TA:

Auscultación cardiopulmonar:

Xantelasmas

Xantomas

Bocio

Hirsutismo

Extremidades:

Varices

Edemas

\section{ESTUDIOS COMPLEMENTARIOS:}

$\begin{array}{llccrcr}\text { Endocrinología: } & \text { No } & \text { Sí, bien } & \text { Sí, problemas } & \text { Dr/a: } \\ \text { Psiquiatría: } & \text { No } & \text { Sí, bien } & \text { Sí, problemas } & \\ \text { Gastroscopia: } & \text { Normal } & \text { Gastritis } & \text { Úlcera } & \text { HP } \\ \text { ECO abdominal: } & & \text { Normal } & \text { Colelitiasis } & \text { Esteatosis } \\ \text { Estudio del sueño: } & & & & \\ \text { ECOcardiograma: } & \text { FE: } & & \end{array}$

Figura 12. Formulario de primera consulta de cirugía bariátrica 
№: Nombre:

Sexo: $\mathrm{H}$ M Edad: № $\mathrm{H}^{\mathrm{a}}$ :

Teléfono:

Fechas: Ingreso:

BIG:

Alta:

\begin{tabular}{|c|c|c|c|c|}
\hline Peso & Peso ideal & SP & Talla & IMC \\
\hline & & & & \\
\hline
\end{tabular}

Colocación de BIG.

Técnica habitual: $\quad$ Balón de silicona con $500 \mathrm{cc}$ de SF+100cc de azul de metileno

Otra técnica:

Cual:

\section{EVOLUCIÓN POSTCOLOCACIÓN DE BIG.}

Sin incidencias:

$\begin{array}{lll}\text { Vómitos: Ninguno } & \text { Moderados } & \text { Muy intensos } \\ \text { Dolor: } & \text { Normal } & \text { Más de lo normal } \\ \text { Otras incidencias } & \text { Cual: } \\ \text { Complicaciones: no } & \text { si Cual: }\end{array}$

Rx de abdomen: Normal Anormal Qué:

Seguimiento (1)

Fecha:

\begin{tabular}{|c|c|}
\hline Peso & IMC \\
\hline & \\
\hline
\end{tabular}

Complicaciones:

Seguimiento (2)

Fecha:

\begin{tabular}{|c|c|}
\hline Peso & IMC \\
\hline & \\
\hline
\end{tabular}

Complicaciones:

Seguimiento (3)

Fecha:

\begin{tabular}{|c|c|}
\hline Peso & IMC \\
\hline & \\
\hline
\end{tabular}

Complicaciones:

\section{Retirada de balón.}

Fecha:

Incidencias:

Cirugía prevista para el día:

Figura 13. Formulario de colocación y seguimiento del balón intragástrico 
Fechas: Ingreso: Cirugía:

Alta:

\begin{tabular}{|c|c|c|c|c|}
\hline Peso & Peso ideal & SP & Talla & IMC \\
\hline & & & & \\
\hline
\end{tabular}

TÉCNICA QUIRÚRGICA

$B P G$

reservorio:............ cm

asa BP $\mathrm{cm}$

asa alimentaria .. $\mathrm{cm}$

asa común $\mathrm{cm}$

\section{BPG laparoscópico}

Gastrectomía vertical

Otra técnica:

Duración: $\min$

Incidentes:

TÉCNICA ASOCIADA

Gastrostomía

Colecistectomía

Hernioplastia

Parietoplastia profiláctica

Gastrectomía estómago excluido

Eventroplastia Esplenectomía

Otra:

\section{EVOLUCIÓN POSTOPERATORIA}

Complicaciones quirúrgicas

No

Dehiscencia:

Hemorragia

Absceso abdominal

Evisceración

\section{Complicaciones médicas}

No

Neumonía

TEP

ITU

otra:

Obstrucción

$\mathrm{IHQ}$

Otra:

EGD: no / si:

Reintervención: no / si

Causa/técnica:

Mortalidad: no / si

Figura 14. Formulario de alta de cirugía bariátrica 
Número protocolo:

Teléfono:

Sexo/F. Nacimiento:

Nombre

$\mathrm{NHC}$

F. LEQ:

F. Ingreso:

F. Intervención:

F. Alta:

IMC $1^{\text {a }} \mathrm{Cta}$ (Peso/Altura)

Peso: $1 \mathrm{~m} \quad 6 \mathrm{~m} \quad 1 \mathrm{a} \quad 2 \mathrm{a}$

Complicaciones que demoraron alta:

Médicas:

Quirúrgicas:

Reingresos:

Patología asociada antes de la cirugía: HTA / DM 1 ó 2 / DL / SAHOS / Artrosis / Otra:

Medicación inclusión LEQ (coste, €):

Medicación antes de la cirugía (coste, €):

\begin{tabular}{|l|l|l|l|l|l|}
\hline $\begin{array}{l}\text { Patología } \\
\mathrm{N}^{\circ} \text { Fcos }\end{array}$ & PreQx & $1 \mathrm{~m}$ & $6 \mathrm{~m}$ & $1 \mathrm{a}$ & $2 \mathrm{a}$ \\
\hline & & & & & \\
\hline & & & & & \\
\hline & & & & & \\
\hline & & & & & \\
\hline & & & & & \\
\hline & & & & & \\
\hline
\end{tabular}

Medicación nueva a los dos años (coste, €):

\begin{tabular}{|l|l|l|l|}
\hline $1 \mathrm{~m}$ & $6 \mathrm{~m}$ & $1 \mathrm{a}$ & $2 \mathrm{a}$ \\
\hline & & & \\
\hline & & & \\
\hline & & & \\
\hline & & & \\
\hline
\end{tabular}

BAROS a los 2 años: $\quad$ Sin Comorbilidades: $\quad$ Con Comorbilidades:

Figura 15. Protocolo de recogida de datos 


\subsection{Criterios y definiciones}

\subsubsection{Criterios de inclusión}

- Edad entre los 18 y 65 años.

- $\quad I M C$ mayor de $35 \mathrm{~kg} / \mathrm{m}^{2}$ con comorbilidades o mayor de $40 \mathrm{~kg} / \mathrm{m}^{2}$.

- Intervención primaria de BPG.

- Fracaso del tratamiento médico, con valoración por Endocrinología y tratamiento por su parte al menos durante un año.

- Ser capaz de entender y asumir los riesgos de la cirugía, con la firma del Consentimiento Informado.

\subsubsection{Criterios de exclusión}

- Patología psiquiátrica que impida comprender la modificación de hábitos dietéticos que implica esta cirugía.

- Fumador activo.

- No consentimiento del paciente.

- Cirugía de revisión.

\subsubsection{Datos de filiación}

Se registró en la primera visita, el número de historia, fecha de nacimiento, el género, fecha de visita y de inclusión en lista de espera quirúrgica (LEQ), dirección y teléfono.

\subsubsection{Variables antropométricas}

\section{a. Talla}

La talla se midió con el paciente en bipedestación y mirando al frente, con los hombros equilibrados y los brazos relajados a lo largo del cuerpo. El cuerpo debía mantener contacto con la barra en cinco puntos: la parte posterior de la cabeza, hombros, glúteos, pantorrillas y talones, con las piernas rectas y los pies planos, perfectamente apoyados en el suelo. La medida se tomaba en $\mathrm{cm}$, hasta el mm más cercano. 


\section{b. Peso corporal}

Para realizar esta medida se colocó la balanza en una superficie perfectamente estable, plana, dura y horizontal. Se le pidió al paciente que se situara justo en medio de la plataforma de la balanza, con los pies ligeramente separados y que permaneciera quieto hasta terminar la medida. El peso corporal se midió en $\mathrm{kg}$, hasta los $100 \mathrm{~g}$ más próximos. Al paciente se le pidió que se quitara el calzado, cualquier objeto pesado (teléfono, monedero, cinturón, etc.) y que se quedara en camiseta y pantalón/falda.

\subsubsection{Comorbilidades relacionadas con la obesidad.}

\section{Antecedentes de riesgo cardiovascular}

En la anamnesis de la visita inicial se recogieron datos sobre antecedentes personales poniendo especial interés en aquellos asociados a la obesidad como son: HTA, DMT2, DL, episodios de TVP y cardiopatías. También se interrogó sobre el grado de actividad física y hábitos tóxicos; el hábito tabáquico es una contraindicación para la cirugía en nuestro Centro. Se realizó una medición de la TA, así como el perímetro de cintura, ambos incluidos en la definición de síndrome metabólico (SM) (152), si bien no son objeto de análisis en este trabajo.

Como parte del reconocimiento de la visita inicial, se realiza una exploración física, para describir el tipo de fenotipo de obesidad que presenta, descartar la presencia de hernias, que sería necesario reparar en el mismo acto quirúrgico, o incisiones de laparotomía que nos hagan pensar en la dificultad del abordaje laparoscópico. Una auscultación cardio-pulmonar y una exploración de miembros inferiores en busca de signos de insuficiencia venosa crónica completaron el procedimiento.

\section{Otras enfermedades asociadas a la obesidad}

También se recogieron antecedentes de SAHS, incontinencia urinaria, hipertensión intracraneal, colelitiasis, varices, amenorrea, artrosis, enfermedad por reflujo gastroesofágico y depresión. 
De todas las patologías asociadas a la obesidad, susceptibles de medicación, se anotó tanto el fármaco como la dosis y frecuencia.

\subsubsection{Cirugía}

Todos los pacientes fueron intervenidos de manera programada en el mismo quirófano del HUCA previa consulta ambulatoria. En ella se les explicó los beneficios y riesgos de la intervención, y firmó un Consentimiento Informado en el que se recoge la posibilidad del uso de los datos generados por la cirugía. Fueron dos los cirujanos principales, ambos presentes en la mayoría de las intervenciones, pero al menos uno de ellos en todas.

Para optimizar a los pacientes de cara a la cirugía realizaron fisioterapia respiratoria, y la semana previa a la intervención tomaron una dieta líquida con suplementos hiperproteicos e hipocalóricos. A aquellos que cumplían criterios se les colocó un BIG los meses anteriores como se ha explicado. Los pacientes ingresaron el día previo a la intervención, que se efectuó habitualmente a primera hora del día siguiente. Al menos las primeras 24 horas siguientes a la intervención las pasaron en Reanimación. Tras un estudio favorable con gastrografín al día siguiente de la intervención, para descartar complicación en la anastomosis gastroyeyunal, fueron trasladados a la habitación. Los días de ingreso se prolongaron hasta garantizar que no existían complicaciones quirúrgicas precoces y que toleraban la dieta necesaria para poder ir de alta.

\section{Procedimiento}

El tipo de BPG fue estándar en la parte gástrica (restrictiva), sobre curvatura menor y adaptado para una capacidad entre $25-50 \mathrm{cc}$. Varió en función del IMC en la parte intestinal (malabsortiva), calibrando la longitud del asa alimentaria (AA) o común (AC) en función del mismo (figura 16). 


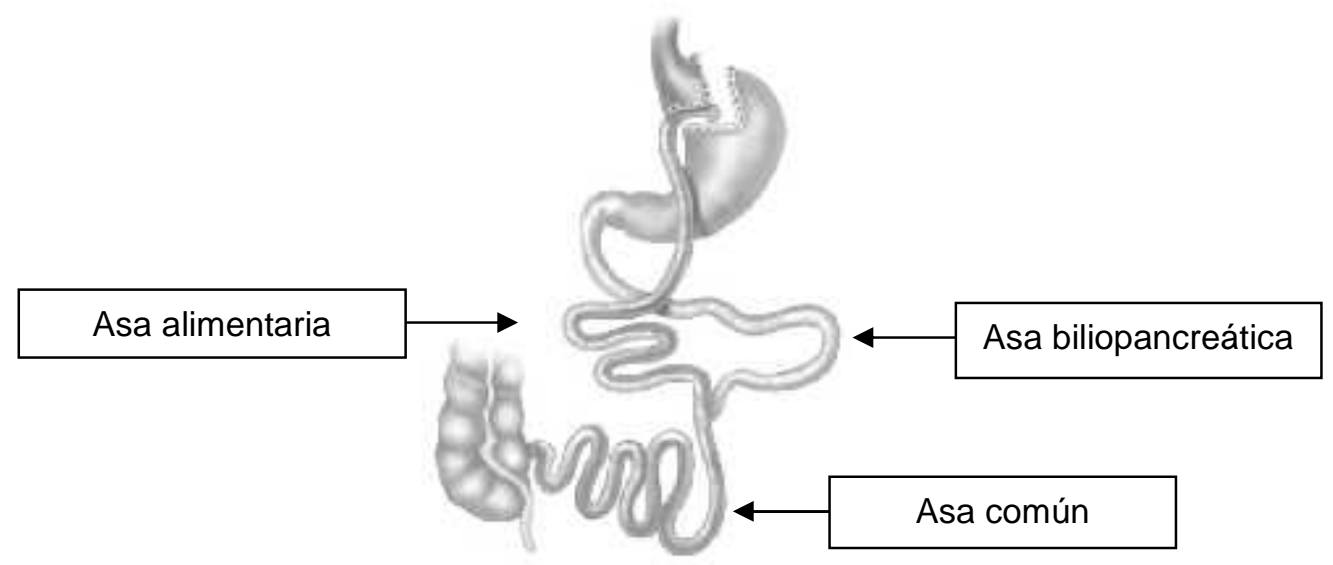

Figura 16. Bypass gástrico. Tipos de asas para su calibrado

En aquellos pacientes con un IMC entre 35 y $45 \mathrm{~kg} / \mathrm{m}^{2}$, se realizó un BPG corto en el que el AA mide $70 \mathrm{~cm}$, el mínimo imprescindible para garantizar que no exista reflujo biliar. Para IMC entre 46 y $55 \mathrm{~kg} / \mathrm{m}^{2}$ realizamos un BPG largo, con AA de $200 \mathrm{~cm}$, siempre que el AC sea mayor de $100 \mathrm{~cm}$. En las obesidades más extremas, es decir con IMC mayor de $55 \mathrm{~kg} / \mathrm{m}^{2}$, indicamos un BPG distal con $100 \mathrm{~cm}$ de $A C$, el mínimo necesario para no incurrir en problemas nutricionales derivados de la malabsorción; añadimos una colecistectomía en todos ellos, por el alto índice de colelitiasis asociado a esta cirugía (153). En todos los casos el asa biliopancreática (ABP) midió $50 \mathrm{~cm}$ (tabla 6).

Tabla 6. Calibrado de asas según IMC

\begin{tabular}{|c|c|c|c|c|c|}
\hline BPG & IMC & ABP & AA & AC & Colecistectomía \\
\hline Corto & $35-45 \mathrm{~kg} / \mathrm{m}^{2}$ & $50 \mathrm{~cm}$ & $70 \mathrm{~cm}$ & El resto & Si colelitiasis \\
\hline Largo & $46-55 \mathrm{~kg} / \mathrm{m}^{2}$ & $50 \mathrm{~cm}$ & $200 \mathrm{~cm}$ & El resto $(>100 \mathrm{~cm})$ & Si colelitiasis \\
\hline Distal & $>55 \mathrm{~kg} / \mathrm{m}^{2}$ & $50 \mathrm{~cm}$ & El resto & $100 \mathrm{~cm}$ & Siempre \\
\hline
\end{tabular}

BPG: Bypass gástrico, IMC: Índice de masa corporal, ABP: Asa biliopancreática, AA: Asa alimentaria, AC: Asa común

La cirugía laparoscópica fue implantándose de manera paulatina desde el año 2007, cuando ya se habían realizado más de 100 BPG abiertos, seleccionando los primeros casos por su IMC, para paulatinamente según se iba superando la curva de aprendizaje, pasar a casos más complejos. Los pacientes con 
obesidades extremas no eran candidatos, al menos en el momento del estudio, a esta vía de abordaje.

\section{Complicaciones}

Entendemos por complicación cualquier desviación del curso postoperatorio normal (154).

a) Sistémicas: Cualquier patología médica sintomática que se produzca en el periodo de ingreso postoperatorio, o que requiera reingreso en los 30 días posteriores al alta.

b) Quirúrgicas: Cualquier patología derivada del procedimiento quirúrgico, en el periodo de ingreso postoperatorio, o que requiera reingreso en los 30 días posteriores al alta.

c) Reintervención: Toda cirugía adicional que precise el paciente durante el postoperatorio, o durante los 30 días posteriores al alta.

\section{Medicación al alta}

En el momento del alta se indicó que reiniciaran la medicación previa al ingreso. En la mayoría de los pacientes diabéticos fue frecuente el ajuste en la medicación, por el efecto inmediato de esta cirugía, ya mencionado. Además se realizó profilaxis de trombosis venosa profunda con heparinas de bajo peso molecular durante 1 mes, y omeprazol hasta acudir a la primera revisión. Como suplementos se pautó un complejo multivitamínico así como hierro oral, en forma de sulfato ferroso por tener mejor absorción tras esta cirugía.

\subsubsection{Seguimiento}

El seguimiento en consulta de todos los pacientes tras la intervención se realizó de acuerdo al protocolo establecido en la tabla 7 , con recogida de datos en el formulario de la figura 17, que se introdujeron en la base de datos. Se puso especial atención a la evolución ponderal, secuelas inherentes al procedimiento quirúrgico, resolución parcial o total de las comorbilidades, y reducción o no de fármacos necesarios para su control (HTA, DM,...) o necesidad de nuevos, 
junto a la adherencia a la terapéutica sustitutiva (vitaminas, minerales,...). En las comorbilidades distinguimos entre: igual, mejorada y curada (cuando el paciente refiere no padecerla y no precisa de medicación). Tanto la primera consulta, como las sucesivas tuvieron lugar en una consulta monográfica de obesidad atendida por cirujanos dedicados a esta patología.

Pasados cinco años de la intervención damos de alta al paciente en el Servicio de Cirugía General, pero deberá mantener el seguimiento por Endocrinología de por vida, por tratarse de una intervención malabsortiva (77). Ante cualquier incidencia relacionada con la cirugía nos remiten de nuevo al enfermo.

En aquellos pacientes que al segundo año de la intervención, momento cuando asumimos que la pérdida ponderal es más o menos estable, tenían un IMC menor de $30 \mathrm{~kg} / \mathrm{m}^{2}$, fueron enviados a Cirugía Plástica para valorar el tratamiento de las secuelas de la CB (abdomen, muslos y brazos). Se realizó la reparación simultánea de eventración en caso de presentarla. Esta cirugía realizada pasados dos años de la cirugía de la obesidad, queda fuera de nuestra evaluación.

En cada revisión anual se realizó un cuestionario BAROS. Para este estudio hemos tomado el cuestionario del segundo año.

Puesto que el cierre del estudio se efectuó en diciembre de 2012, todos los pacientes mantuvieron un seguimiento mínimo de dos años.

Tabla 7. Revisiones y pruebas del seguimiento de cirugía bariátrica

\begin{tabular}{|c|c|c|c|c|c|c|c|}
\hline & 1 mes & 6 meses & 1 año & 2 años & 3 años & 4 años & 5 años \\
\hline $\mathrm{H}^{\mathrm{a}}$ y exploración & * & * & * & * & * & * & * \\
\hline ECO abdominal & & & * & * & * & * & * \\
\hline Contraste & & * & * & * & * & * & * \\
\hline Gastroscopia & \multicolumn{7}{|c|}{ Según clínica } \\
\hline Analítica & \multicolumn{7}{|c|}{ Revisar las de Nutrición } \\
\hline
\end{tabular}


$\mathrm{NH}$ ㅇ: Nombre:

\begin{tabular}{|l|l|l|l|}
\hline Fecha revisión & $/$ & Fecha intervención & $/$ \\
\hline Tipo intervención & \multicolumn{3}{|l|}{} \\
\hline
\end{tabular}

Peso: inicial $\mathrm{kg}$

actual $\mathrm{kg}$

Clínica:

- diarrea

- vómitos

- hemorragia

- dolor abdominal

- eventración

- anemia

- desnutrición - déficits:

- otra:

\section{Comorbilidades:}

$\begin{array}{llll}\text { - HTA } & \text { - DM } & \text { - Varices } & \text { - Incontinencia urinaria } \\ \text { - Cardiopatía } & \text { - Apnea del sueño } & \text { - Colelitiasis } & \text { - Amenorrea } \\ \text { - Dislipemia } & \text { - Artrosis } & \text { - Depresión } & \text { - HT intracraneal }\end{array}$

- ERGE

Tratamiento medicamentoso actual:

Estudios complementarios:

\begin{tabular}{|c|c|c|c|c|c|}
\hline Gastroscopia: & no & Normal & Úlcera & Estenosis & Comunicación Gastro-Gástrica \\
\hline Contraste: & no & Normal & Úlcera & Estenosis & Comunicación Gastro-Gástrica \\
\hline ECO abdominal: & & no & Normal & Colelitiasis & Otra \\
\hline Dilataciones: & & no & si: & & \\
\hline
\end{tabular}

Otros:

Reintervención: no si fecha:

Causa/ técnica:

Figura 17. Formulario de seguimiento de cirugía bariátrica 


\subsection{Cálculo de costes}

\subsubsection{Contabilidad analítica mediante GRD}

Se solicitó al Servicio de Admisión la codificación de todos los pacientes a partir de su número de historia y fecha de ingreso, cirugía y alta. Nos devolvieron el GRD en el que fueron agrupados cada uno de los episodios de ingreso, tras completarse la CMBD al alta. Como ya se explicó tenemos tres tipos de GRD quirúrgicos para toda la serie en el ingreso de la cirugía, así como otros dos quirúrgicos correspondientes a dos reingresos y otro médico en aquellos que precisaron BIG preoperatorio. El peso de los GRDs se estableció según la versión 27.0, utilizada en el HUCA para el año 2013, y el coste asignado por unidad de peso es el derivado del GFH de Cirugía General con Hospitalización del HUCA.

\subsubsection{Cálculo individualizado}

Este cálculo, mucho más complejo pero más preciso, resulta del desglose de cada una de las fases del ingreso. Por una parte calculamos el coste del ingreso por días de estancia hospitalaria, al que añadimos el coste del acto quirúrgico (recuperando el tiempo empleado en cada una de las intervenciones, de los datos registrados en la codificación quirúrgica y facilitado por el Servicio de Control de Gestión del hospital), y sumamos el tiempo de quirófano, de cirujanos, de anestesistas y de material empleado. Dado que es una cirugía estandarizada realizada por un grupo reducido de cirujanos y con evolución postoperatoria similar en la mayoría de los casos, hicimos un cálculo estándar de la medicación que precisará en el postoperatorio y todo lo que se salga de este estándar, por el que pasarán todos los pacientes a lo largo del ingreso, se sumará cada día de ingreso extra por la media de gasto farmacéutico imputado al Servicio de Cirugía General en el año 2013. Como todos los pacientes precisan una serie de estudios preoperatorios y postoperatorios, así como un número establecido de consultas de los distintos servicios implicados, también calculamos el coste de estas variables y lo agregamos individualmente. En los pacientes que precisaron un BIG se les sumó el coste del ingreso para su colocación teniendo en cuenta el preoperatorio, el tiempo empleado en el área 
quirúrgica con un anestesista, un endoscopista y un médico residente, así como una gastroscopia ambulatoria para su retirada y las revisiones para ver la evolución ponderal durante los cuatro meses que lo llevan. Todos estos datos fueron cuantificados y asignados a cada uno de los pacientes por separado.

También para el cálculo individualizado tomamos como referencia de costes el año 2013, ya que cuando los costes y efectos se producen en un periodo superior al año, es preciso transformarlos en las unidades equivalentes a las del año cero (momento en que se realiza la evaluación), que correspondería al año 2014 pero como este fue el año que se produjo el traslado del HUCA a su actual ubicación no disponemos de datos fiables; y en el momento del estudio aún no se había cerrado el ejercicio del 2015.

\section{Coste de Personal}

El coste de personal facultativo se asignó al paciente teniendo en cuenta los siguientes criterios:

El coste de personal es medido en tiempo efectivo de trabajo en la realización de las actividades cotidianas y según los minutos de trabajo total en el mes.

El coste medio hora del personal facultativo en un determinado GFH, se obtuvo:

- Coste hora $=$ Coste total del personal facultativo / N. total de minutos trabajados.

La asignación del coste al episodio clínico de los distintos facultativos, diferenciando entre médico interno residente (MIR) y facultativo especialista de área (FEA), que intervienen en la realización de una intervención quirúrgica se calculó:

- Coste Cirujano $=$ Tiempo de intervención $\times$ Coste medio minuto Facultativo $\times \mathrm{N}$. - de cirujanos que intervinieron en la operación (diferenciando MIR y FEA).

- Coste Anestesista $=$ Tiempo de anestesia $\times$ Coste medio Anestesista $\mathrm{x}$ N. de Anestesistas que intervienen en la operación (diferenciando MIR y FEA). 
- Coste Digestólogo/Endoscopista $=$ Tiempo de intervención $\times$ Coste medio minuto Facultativo $\times$ N.․ de digestólogos que intervienen en la endoscopia en quirófano (diferenciando MIR y FEA).

\section{Coste de Farmacia}

El coste del consumo de los distintos medicamentos que se suministran a un paciente se asigna al episodio clínico siempre que la dispensación se realice a través del sistema en dosis unitaria. En este trabajo partimos de un periodo estándar de ingreso en el que calculamos el coste de toda la medicación dispensada para un periodo de seis días, según el protocolo de la Unidad, y a partir de este número de días lo que hacemos es multiplicar los días de más por la media de gasto farmacéutico por día de nuestro Servicio. De este modo asumimos que en algún momento del ingreso todos los pacientes pasan por las fases calculadas en el protocolo.

\section{Coste de Suministros Específicos}

El material que supone un coste muy importante y constituye un elemento diferencial de un proceso se asigna al episodio clínico. En este caso se calculó el gasto generado para cada una de las intervenciones quirúrgicas atendiendo al abordaje abierto o laparoscópico, considerando si hubo BIG previo o si se realizó colecistectomía, cuantificando todo el material empleado. La tarea se facilita al tratarse de una cirugía muy estandarizada, en la que el consumo de recursos es prácticamente igual en todos los pacientes. En caso de complicación y reintervención se realizó a su vez un cálculo individualizado.

\section{Costes de Anatomía Patológica}

El coste de la producción realizada por el GFH de Anatomía Patológica se asigna directamente al episodio clínico según el catálogo de pruebas y el coste de las pruebas realizadas. Lo aplicamos en el caso de existir colecistectomía asociada. 


\section{Coste de Laboratorios}

El coste de la producción realizada por los distintos laboratorios (Análisis Clínicos, Hematología, Microbiología, etc....) se asigna directamente al episodio clínico según el catálogo de pruebas y el coste de las pruebas realizadas. En nuestro caso lo tomamos del catálogo del Servicio Vasco de Salud al no tener la posibilidad de obtener este coste de nuestro Centro, y asumir que se trata de un sistema de salud equiparable.

\section{Coste de las Unidades de Enfermería}

El coste generado por las Unidades de Enfermería se asignó directamente al episodio clínico según las estancias generadas.

\section{Coste de Quirófanos}

El coste del GFH quirófano se imputó a cada episodio clínico en función de los minutos utilizados para cada intervención. En él se incluyeron tanto costes directos: medicación dispensada en quirófano, personal no facultativo del Área Quirúrgica y el material utilizado por parte de Anestesia, excluyendo el material sanitario asignado de manera independiente; así como costes indirectos repercutidos de otros Servicios intermedios (farmacia, lavandería, banco de sangre, mantenimiento,...) y estructurales. El coste de una hora de intervención se ha obtenido dividiendo los costes agregados por el número total de horas utilizadas, las cuales conocemos a través del registro de Control de Gestión que recoge mensualmente el número de horas que cada Servicio consume de quirófano.

\section{Coste de Reanimación}

El coste total del GFH Reanimación, se dividió por el número de intervenciones realizadas y el resultado se asignó a cada episodio clínico, ya que no se conoce el tiempo de reanimación correspondiente a cada paciente. 


\section{Coste de Radiodiagnóstico}

El coste de la producción de Radiodiagnóstico se asigna directamente al episodio clínico según el catálogo de exploraciones y el coste de las exploraciones realizadas.

\section{Coste de Alimentación}

El coste de la producción realizada de Alimentación se asignó directamente al episodio clínico según el catálogo de dietas y el coste de las dietas y las ingestas realizadas.

\subsubsection{Costes recogidos}

Los costes más interesantes serán los derivados del ingreso, en el que se incluyen tanto los propios de la cirugía como los de hospitalización, para poder compararlos por los proporcionados por los GRDs y por las distintas técnicas quirúrgicas, pues el resto de costes calculados tanto de consultas como de estudios pre y postoperatorios son similares para todos los pacientes. Sumamos al coste del ingreso para la cirugía los costes de la colocación del BIG previo por paciente y por GRD (ya que requiere ingreso).

De los costes en fármacos de los pacientes en cada una de las visitas postoperatorias queda recogida la medicación, teniendo cuatro momentos de actualización de la misma (al mes, seis meses, año y dos años). El coste de los medicamentos analizados en un principio los obtuvimos del catálogo de información sobre los productos incluidos en la prestación farmacéutica del SNS del Ministerio (155). Debido a la gran variabilidad de medicamentos y modificación de los mismos en las distintas revisiones optamos por tomar como coste de los mismos los obtenidos de publicaciones de nuestro país. Así queda recogido a continuación el coste anual en fármacos por patologías: 
- HTA: De acuerdo con Sicras (156) el coste anual del tratamiento en fármacos de la HTA es de 894€. Tomaremos esta referencia a la hora de hacer el cálculo del ahorro que supone su resolución.

- DM: Tomando como referencia los resultados de Crespo (157), el coste estimado en fármacos en nuestro país es de $265 €$ al año.

- DL: Con los resultados recogidos de Oliva (158), asumimos que el coste anual en fármacos es de $282 €$.

- SAHS: El coste de la terapia CPAP es de unos $675 €$ anuales $(1,85 €$ por sesión), cantidad que utilizaremos en nuestro cálculo (159).

- Depresión: La diferencia de coste entre pacientes que presentan remisión de esta patología, respecto a los que no, se estima en los $1.000 €$ anuales, cifra que tomaremos de referencia (160).

Con estos valores haremos el cálculo del ahorro que supone en nuestro entorno la resolución de comorbilidades tras la cirugía de la obesidad, y como repercute en el gasto farmacológico.

En el resto de patologías no realizaremos una estimación de reducción de costes por no disponer de estudios adecuados para conocer el coste de cada una de ellas. 


\subsection{Análisis estadístico}

Revisión de los protocolos de inclusión y seguimiento de la Unidad de Cirugía Bariátrica, así como la historia clínica de los pacientes, con un seguimiento de dos años, que es el tiempo considerado de referencia para alcanzar la mayor pérdida ponderal. En colaboración con el Servicio de Contabilidad de Gestión del HUCA, que nos proporcionó el coste que supuso cada una de las intervenciones, calculado mediante contabilidad analítica tanto por GRDs, como por paciente.

El análisis fue realizado por el encargado del área de estadística del HUCA.

El asesoramiento de la metodología de estudio y supervisión de la parte económica lo realizó el Servicio de Contabilidad de Gestión del HUCA. La Oficina de Investigación Biosanitaria (OIB) del Principado de Asturias a su vez prestó asesoramiento metodológico.

Metodología estadística: Estudio observacional, descriptivo, longitudinal y retrospectivo sobre base de datos prospectiva. Se realizó el análisis estadístico mediante el software estadístico de libre distribución R (www.r-project.org) (161). Realizamos estadística descriptiva, expresando las variables continuas en forma de media (M), desviación estándar (DE), y en algunas se detalla la mediana y el rango. Las variables categóricas están expresadas como frecuencias y porcentajes.

La comparación por grupos se realizó mediante la prueba robusta de Welch, cuando las variables fueron simétricas, y mediante test no-paramétricos de Kruskal-Wallis cuando las variables fueron asimétricas. La comparación entre variables categóricas se realizó mediante la prueba exacta de Chi-cuadrado.

Nivel de significación: Consideramos diferencias significativas a las $p<0,05$.

Variables de estudio: Descritas en el apartado 5.3. 


\subsection{Análisis coste-efectividad}

Para conocer la relación coste-efectividad primero definimos los datos de efectividad, que a nuestro juicio giran en torno a tres ámbitos: la pérdida de peso, la resolución de comorbilidades, y la calidad de vida, es decir los objetivos que persigue esta intervención; así como analizamos también cada una de estas variables por separado para definir el paciente que mejor las representa. Posteriormente dividimos el coste de cada intervención por la medida utilizada de efectividad, calculado individualmente para todos los pacientes de la muestra, y el cociente obtenido nos muestra la relación costeefectividad. El resultado, en criterio de coste-efectividad, nos indica que la mejor opción terapéutica es aquella con menor cociente, es decir, menos es más.

De esta intervención debemos esperar lo primero y más evidente, la pérdida de peso, valorada mediante el PSP o PEIMCP, con distintos criterios a la hora de definir éxito. Tomamos como referencia el PSP mayor del 50\% para no hablar de fracaso, así en nuestro estudio nos fijamos en los resultados que se recogen en el cuestionario de calidad de vida BAROS: fue buen resultado aquel con un PSP comprendido entre $50 \%$ y $75 \%$, tomando la referencia de los dos años como ya expusimos previamente, y un resultado excelente un PSP mayor del $75 \%$. Para el análisis CE usamos el resultado final, sin agrupar.

Otra de las variables a tener en cuenta a la hora de valorar la efectividad del tratamiento quirúrgico es la resolución de las comorbilidades, ya que son estas las que condicionan tanto la calidad de vida de los pacientes, como su esperanza de vida y los costes asociados. En este sentido, y siguiendo con el modelo propuesto en el BAROS, diferenciamos entre aquellos pacientes en los que se han resuelto todas las comorbilidades mayores y otras mejoradas para definir un resultado excelente, aquellas en las que se resolvió una mayor y otras mejoradas o simplemente mejoradas como resultado bueno, y finalmente aquellos en los que no existe mejoría o se agravan, como fracaso. Hay que apuntar que existe un grupo, definido como obesos metabólicamente sanos (metabolically healthy but obese MHO) (162) y que no presentan 
comorbilidades mayores, en los que no consideraremos esta variable para definir efectividad.

Por último evaluamos el resultado del cuestionario de calidad de vida, sobre una puntuación máxima de 3 puntos y mínima de -3 . Consideramos fracaso un resultado negativo, un buen resultado aquel comprendido entre 0 y 1,5 y excelente por encima de 1,5.

Si juntamos cada uno de estos parámetros, e incluimos los pacientes que presentaron complicación postoperatoria y los que precisaron reintervención, en detrimento de esta puntación, según se indica en el cuestionario de BAROS, tendremos una puntuación final en función de si presentaban comorbilidades previamente o no (tabla 8), que utilizaremos de manera global, ya que incluye los tres aspectos, para definir efectividad.

Tabla 8. Evaluación final de resultados según el cuestionario BAROS

\begin{tabular}{|l|c|c|}
\hline & Sin comorbilidades & Con comorbilidades \\
\hline Fallo & 0 ó menos & 1 ó menos \\
\hline Regular & $>0-1,5$ & $>1-3$ \\
\hline Buena & $>1,5-3$ & $>3-5$ \\
\hline Muy buena & $>3-4,5$ & $>5-7$ \\
\hline Excelente & $>4,5-6$ & $>7-9$ \\
\hline
\end{tabular}

En un principio planteamos un análisis estadístico de regresión logística, transformamos la variable de PSP a los dos años en dos grupos, excelente frente a los demás. A partir de aquí lo comparamos con el resto de variables susceptibles de estudio. Finalmente se desestimó por las características de la serie y métodos de definición de efectividad dicotómicos, que tras realizar alguna aproximación, a criterio del estadístico, no ofrecía suficiente solvencia.

Hicimos un análisis diferenciado de los pacientes con DM, considerados por múltiples publicaciones como los grandes beneficiados de esta cirugía $(128,129,131,163,164)$. 
RESULTADOS 



\section{RESULTADOS}

\subsection{Estudio descriptivo preoperatorio}

\subsubsection{Cohorte a estudio}

De los 384 pacientes intervenidos en el HUCA desde que se inició el programa de cirugía bariátrica hasta diciembre de 2012, se excluyeron 77 de acuerdo a la figura 18.

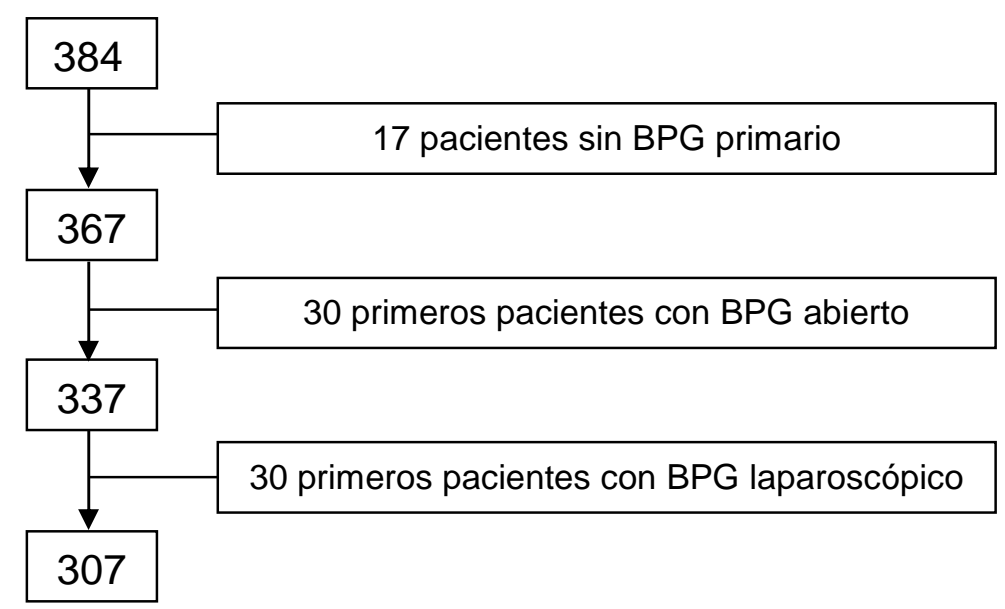

Figura 18. Diagrama de la selección de pacientes incluidos en el estudio 


\subsubsection{Características de la cohorte}

Las características generales de los pacientes incluidos en el estudio se muestran en la tabla 9.

Tabla 9. Características generales de la serie

\begin{tabular}{|l|l|}
\hline Variable (n=307) & \\
\hline Edad: media (DE), mediana (rango), años & $43,5(10), 43,5(18,1-62,2)$ \\
\hline Sexo: Hombre/Mujer (\%) & $68(22,1) / 239(77,8)$ \\
\hline Talla: media (DE), mediana (rango), cm & $162,3(8,5), 162(146-186)$ \\
\hline Peso (1 a Consulta): media (DE), mediana (rango), kg & $131,8(21,4), 127(95-219)$ \\
\hline Peso (Preoperatorio): media (DE), mediana (rango), kg & $127,3(17,8), 125(89-207)$ \\
\hline IMC (1 ${ }^{\text {a }}$ Consulta): media (DE), mediana (rango), $\mathrm{kg} / \mathrm{m}^{2}$ & $49,9(6,8), 49,5(36,2-77,6)$ \\
\hline IMC (Preoperatorio): media (DE), mediana (rango), $\mathrm{kg} / \mathrm{m}^{2}$ & $48,2(5,3), 48,8(35-64,5)$ \\
\hline Comorbilidades (\%) & $283(92,2)$ \\
\hline HTA (\%) & $164(53,4)$ \\
\hline DM (\%) & $85(27,7)$ \\
\hline DL (\%) & $73(23,7)$ \\
\hline SAHS (\%) & $178(58)$ \\
\hline Artrosis (\%) & $107(34,9)$ \\
\hline Depresión (\%) & $123(40,1)$ \\
\hline ERGE (\%) & $77(25,1)$ \\
\hline Colelitiasis (\%) & $51(16,6)$ \\
\hline Incontinencia urinaria (\% mujeres) & $80(33,4)$ \\
\hline Amenorrea (\% mujeres) & $35(14,6)$ \\
\hline
\end{tabular}

DE: Desviación estándar. IMC: Índice de masa corporal. HTA: Hipertensión arterial. DM: Diabetes mellitus. DL: Dislipemia. SAHS: Síndrome de apnea e hipopnea del sueño. ERGE: Enfermedad por reflujo gastroesofágico. 


\section{Parámetros demográficos}

La población a estudio tiene una edad media (DE) de 43,5 (10) años. Por sexo, 239 son mujeres $(77,8 \%)$ (figura 19$)$.

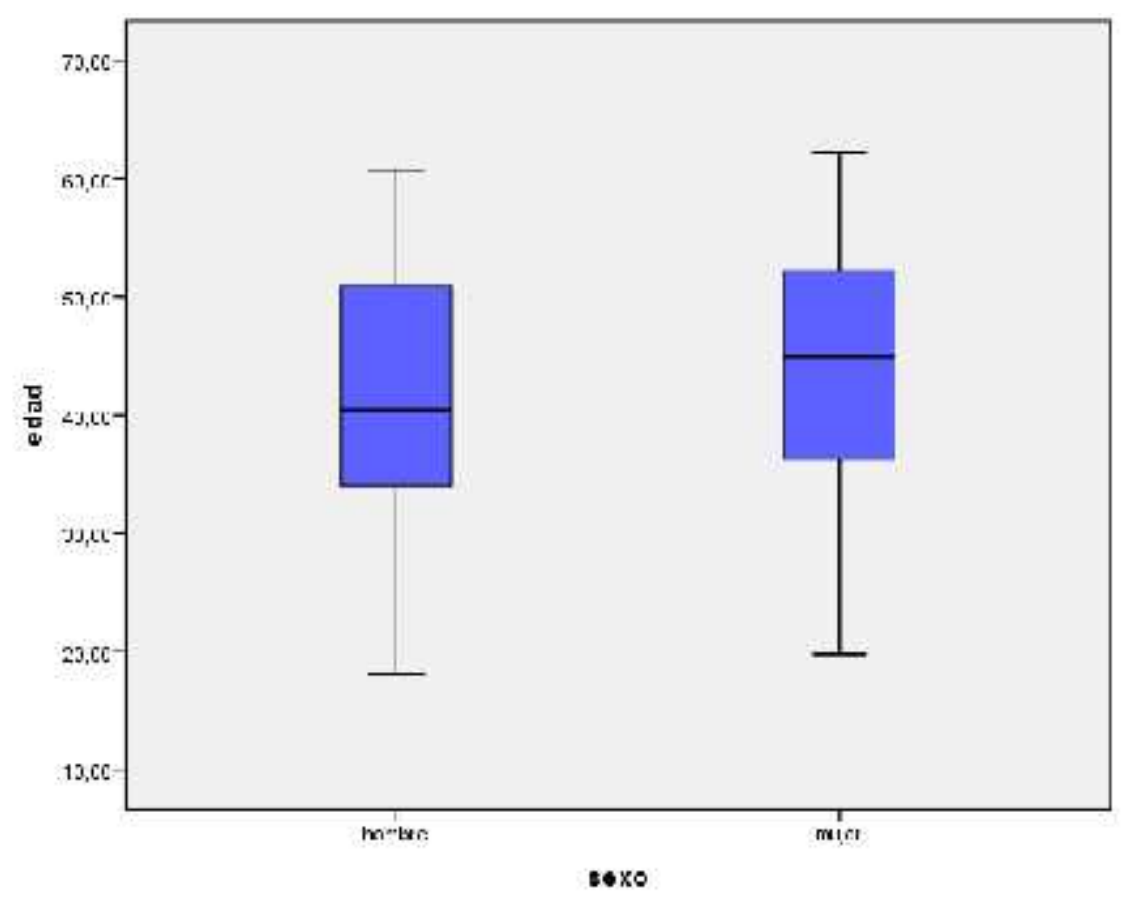

Figura 19. Diagrama de cajas por edad y sexo

\section{Parámetros antropométricos}

La talla media fue de $162,3 \mathrm{~cm}$. La media de peso en la primera consulta fue de $131,8(21,4) \mathrm{kg}$, mientras que la preoperatoria fue de $127,3(17,8) \mathrm{kg}$. El IMC medio en consulta fue $49,9(6,8) \mathrm{kg} / \mathrm{m}^{2}$ y el preoperatorio $48,2(5,3) \mathrm{kg} / \mathrm{m}^{2}$. Si filtramos los pacientes a los que se les colocó un BIG preoperatorio, nos queda un peso en primera consulta de $127,3(17,7) \mathrm{kg}$ y preoperatorio de $125,8(16,5)$ kg. Por IMC $48(5,3) \mathrm{kg} / \mathrm{m}^{2}$ y 47,5 $(4,9) \mathrm{kg} / \mathrm{m}^{2}$, respectivamente. 


\section{Comorbilidades relacionadas con la obesidad}

$283(92,2 \%)$ de los pacientes a estudio presentaban algún tipo de comorbilidad. Entre las comorbilidades mayores relacionadas con la obesidad (figura 20): HTA 164 (53,4\%), DM 85 (27,7\%), DL 73 (23,7\%), SAHS 178 (58\%), Artrosis 107 (34,9\%). De las menores: Depresión 123 (40,1\%), Varices 96 (31,3\%), Incontinencia urinaria $81(26,4 \%)$, ERGE 77 (25,1\%), Colelitiasis $51(16,6 \%)$, Amenorrea 35 (14,6\% de las mujeres), Hipertensión intracraneal idiopática 2 $(0,7 \%)$. Otros: Hipotiroidismo 13, Fibromialgia 7, Trombosis venosa 4 (2 profundas y 2 superficiales). 24 pacientes $(7,8 \%)$ no presentaban ninguna comorbilidad. En la tabla 10 quedan diferenciadas por sexos y en la tabla 11 por IMC.

Tabla 10. Características de la población en función del género

\begin{tabular}{|l|c|c|c|}
\hline Variable (n=307) & Mujeres (n=239) & Hombres (n=68) & $\mathbf{p}$ \\
\hline Edad, media (DE), años & $44(9,8)$ & $41,8(10,3)$ & 0,124 \\
\hline Talla, media (DE), cm & $159(0,06)$ & $172(0,06)$ & $<0,001$ \\
\hline Peso, media (DE), kg & $126,7(17,2)$ & $149,6(24,9)$ & $<0,001$ \\
\hline IMC, media (DE), kg/m² & $49,9(6,4)$ & $50(7,9)$ & 0,944 \\
\hline HTA (\%) & $123(51,5)$ & $41(60,3)$ & 0,217 \\
\hline DM (\%) & $65(27,2)$ & $20(29,4)$ & 0,719 \\
\hline DL (\%) & $52(21,8)$ & $23(33,8)$ & 0,054 \\
\hline SAHS (\%) & $122(51)$ & $56(82,4)$ & $<0,001$ \\
\hline Artrosis (\%) & $88(36,8)$ & $19(27,9)$ & 0,196 \\
\hline Depresión (\%) & $106(44,4)$ & $17(25)$ & 0,005 \\
\hline ERGE (\%) & $60(25,1)$ & $17(25)$ & 1 \\
\hline Colelitiasis (\%) & $44(18,4)$ & $7(10,3)$ & 0,140 \\
\hline
\end{tabular}

DE: Desviación estándar. IMC: Índice de masa corporal. HTA: Hipertensión arterial. DM: Diabetes mellitus. DL: Dislipemia. SAHS: Síndrome de apnea e hipopnea del sueño. ERGE: Enfermedad por reflujo gastroesofágico. 


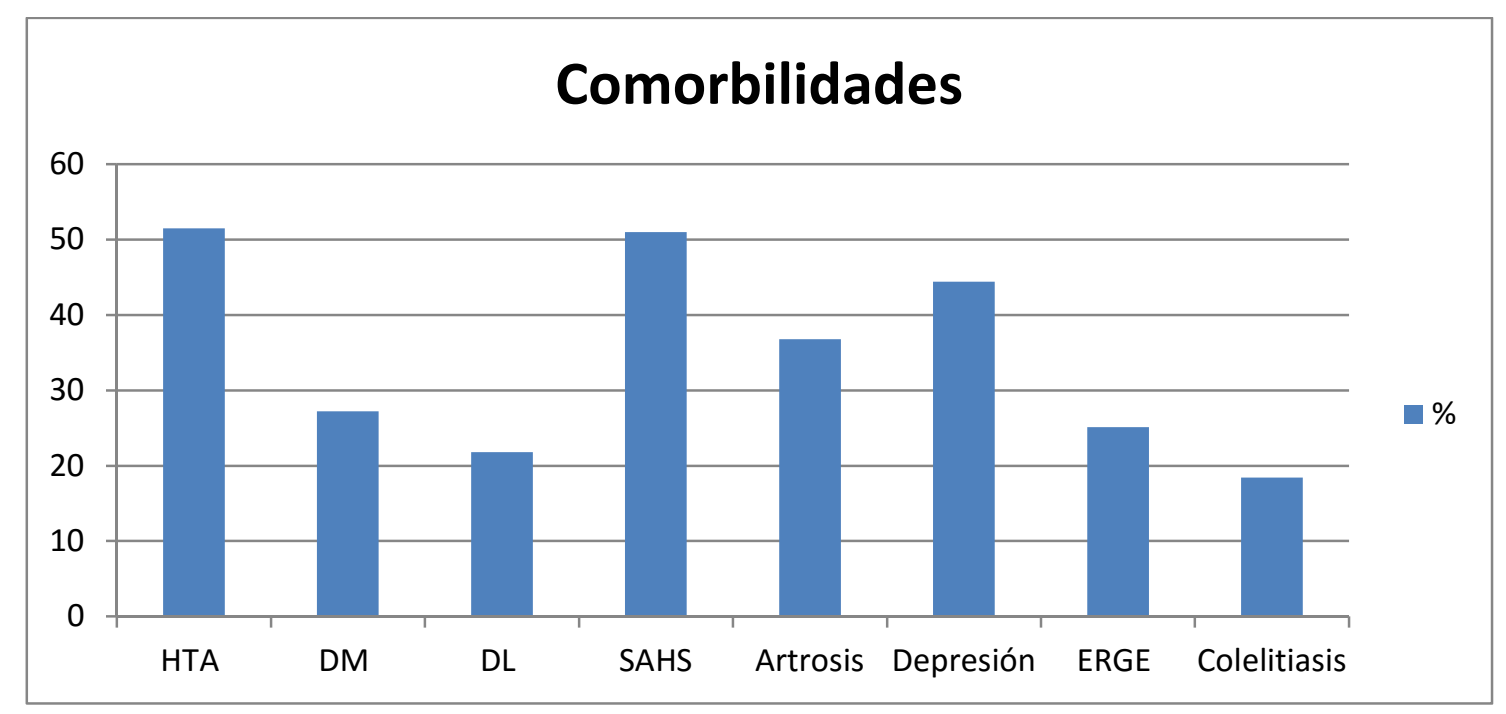

Figura 20. Comorbilidades principales relacionadas con la obesidad

Según el IMC antes de la cirugía, que determina el tipo de bypass que realizamos, distinguimos los siguientes grupos recogidos en tablas.

Tabla 11. Características de la población en función del IMC de la primera consulta

\begin{tabular}{|c|c|c|c|c|}
\hline Variable $(n=307)$ & $\begin{array}{l}\text { IMC 35-45 } \\
(n=76)\end{array}$ & $\begin{array}{l}\text { IMC 46-55 } \\
(n=169)\end{array}$ & $\begin{array}{c}\text { IMC }>55 \\
(n=62)\end{array}$ & $\mathbf{p}$ \\
\hline Edad, media (DE), años & $43,4(8,9)$ & $43,6(10,8)$ & $43,4(8,9)$ & 0,987 \\
\hline $\begin{array}{llll}\text { Sexo: Hombre } & (\%) & \\
\text { Mujer }(\%) & \end{array}$ & $\begin{array}{l}15(19,7) \quad / \\
61(80,2)\end{array}$ & $\begin{array}{lrl}38 & (22,5) & \\
131 & (77,5)\end{array}$ & $\begin{array}{ll}15 & (24,1) \quad / \\
47 & (75,8)\end{array}$ & 0,514 \\
\hline Talla, media (DE), cm & $164,8(0,08)$ & $161,6(0,08)$ & $161,1(0,08)$ & 0,009 \\
\hline Peso, media (DE), kg & $114,7(10,9)$ & $130,2(14,8)$ & $157,1(22,8)$ & $<0,001$ \\
\hline IMC, media (DE), $\mathrm{kg} / \mathrm{m}^{2}$ & $42,1(2)$ & $49,7(2,7)$ & $60,2(4,7)$ & $<0,001$ \\
\hline HTA (\%) & $36(47,4)$ & $93(55)$ & $35(56,5)$ & 0,467 \\
\hline DM (\%) & $21(27,6)$ & $47(27,8)$ & $17(27,4)$ & 0.998 \\
\hline $\mathrm{DL}(\%)$ & $20(26,3)$ & $46(27,2)$ & $9(14,5)$ & 0,125 \\
\hline SAHS (\%) & $39(51,3)$ & $100(59,2)$ & $39(62,9)$ & 0,350 \\
\hline Artrosis (\%) & $25(32,9)$ & $58(34,4)$ & $24(38,7)$ & 0,757 \\
\hline Depresión (\%) & $33(43,4)$ & $65(38,5)$ & $25(40,3)$ & 0,764 \\
\hline ERGE (\%) & $29(38,2)$ & $33(19,5)$ & $15(24,2)$ & 0,008 \\
\hline Colelitiasis (\%) & $12(15,8)$ & $31(18,3)$ & $8(12,9)$ & 0,601 \\
\hline
\end{tabular}

DE: Desviación estándar. IMC: Índice de masa corporal. HTA: Hipertensión arterial. DM: Diabetes mellitus. DL: Dislipemia. SAHS: Síndrome de apnea e hipopnea del sueño. ERGE: Enfermedad por reflujo gastroesofágico. 
Si tomamos el IMC del momento de la cirugía, una vez que se han realizado las medidas previas a la cirugía de colocación de BIG y dieta hipocalórica, nos queda la siguiente tabla.

Tabla 12. Características de la población en función del IMC preoperatorio

\begin{tabular}{|c|c|c|c|c|}
\hline Variable $(n=307)$ & $\begin{array}{l}\text { IMC 35-45 } \\
\qquad(\mathrm{n}=90)\end{array}$ & $\begin{array}{l}\text { IMC 46-55 } \\
(n=188)\end{array}$ & $\begin{array}{c}\text { IMC >55 } \\
(n=29)\end{array}$ & $\mathbf{p}$ \\
\hline Edad, media (DE), años & $43,4(9,1)$ & $43,6(10,5)$ & $42,9(9,3)$ & 0,929 \\
\hline $\begin{array}{l}\text { Sexo: Hombre (\%) / } \\
\text { Mujer (\%) }\end{array}$ & $\begin{array}{lll}24 & (26,7) \quad / \\
66 & (73,3)\end{array}$ & $\begin{array}{lll}38 & (20,2) & / \\
150 & (79,8)\end{array}$ & \begin{tabular}{lcc|}
6 & $(20,7) \quad /$ \\
23 & $(79,3)$ &
\end{tabular} & 0,470 \\
\hline Talla, media (DE), cm & $165(0,08)$ & $161,4(0,08)$ & $159,9(0,09)$ & 0,003 \\
\hline Peso, media (DE), kg & $119,5(15,6)$ & $134,3(18,8)$ & $153,7(28,8)$ & $<0,001$ \\
\hline IMC, media (DE), kg/m² & $43,8(4,2)$ & $51,3(4,9)$ & $59,7(7,1)$ & $<0,001$ \\
\hline HTA (\%) & $46(51,1)$ & $102(54,3)$ & $16(55,2)$ & 0,869 \\
\hline DM (\%) & $24(26,7)$ & $55(29,3)$ & $6(20,7)$ & 0,610 \\
\hline $\mathrm{DL}(\%)$ & $24(26,7)$ & $46(24,5)$ & $5(17,2)$ & 0,590 \\
\hline SAHS (\%) & $50(55,6)$ & $111(59)$ & $17(58,6)$ & 0,857 \\
\hline Artrosis (\%) & $32(35,6)$ & $68(36,2)$ & $7(24,1)$ & 0,443 \\
\hline Depresión (\%) & $37(41,1)$ & $75(39,9)$ & $11(37,9)$ & 0.952 \\
\hline ERGE (\%) & $31(34,4)$ & $43(22,9)$ & $3(10,3)$ & 0,018 \\
\hline Colelitiasis (\%) & $13(14,4)$ & $31(16,5)$ & $7(24,1)$ & 0,474 \\
\hline
\end{tabular}

DE: Desviación estándar. IMC: Índice de masa corporal. HTA: Hipertensión arterial. DM: Diabetes mellitus. DL: Dislipemia. SAHS: Síndrome de apnea hipopnea del sueño. ERGE: Enfermedad por reflujo gastroesofágico.

\section{Número de fármacos según patología antes de la cirugía}

Por patologías, como se recoge en la tabla 13 , vemos el número de fármacos diferentes que se consumen diariamente. 
Tabla 13. Número de fármacos por patología

\begin{tabular}{|l|c|c|c|c|c|}
\hline & Sin fármacos & $\mathbf{1}$ & $\mathbf{2}$ & $\mathbf{3}$ & $\mathbf{4}$ \\
\hline $\begin{array}{l}\text { HTA (\%) } \\
\mathrm{n}=163\end{array}$ & $20(12,3)$ & $91(55,8)$ & $34(20,9)$ & $14(8,6 \%)$ & $4(2,5)$ \\
\hline $\begin{array}{l}\mathrm{DM}(\%) \\
\mathrm{n}=85\end{array}$ & $20(23,5)$ & $39(45,9)$ & $20(23,5)$ & $6(7,5)$ & - \\
\hline $\begin{array}{l}\mathrm{DL}(\%) \\
\mathrm{n}=73\end{array}$ & $38(52,1)$ & $32(43,8)$ & $3(4,1)$ & - & - \\
\hline $\begin{array}{l}\text { Artrosis (\%) } \\
\mathrm{n}=83\end{array}$ & $19(22,9)$ & $48(57,8)$ & $11(13,3)$ & $4(4,8)$ & $1(1,2)$ \\
\hline $\begin{array}{l}\text { Depresión (\%) } \\
\mathrm{n}=121\end{array}$ & $23(19)$ & $47(38,8)$ & $38(31,4)$ & $11(9,1)$ & $2(1,7)$ \\
\hline
\end{tabular}

HTA: Hipertensión arterial. DM: Diabetes mellitus. DL: Dislipemia.

En los pacientes con HTA el número de fármacos varió desde 0 a 4: Ninguno 20 (12,3\%), Uno 91 (55,8\%), Dos 34 (20,9\%), Tres 14 (8,6\%), Cuatro 4 (2,5\%). Mediana: 1. El total de pacientes en los que se recoge este dato es 163 , un $98,7 \%$ de los hipertensos.

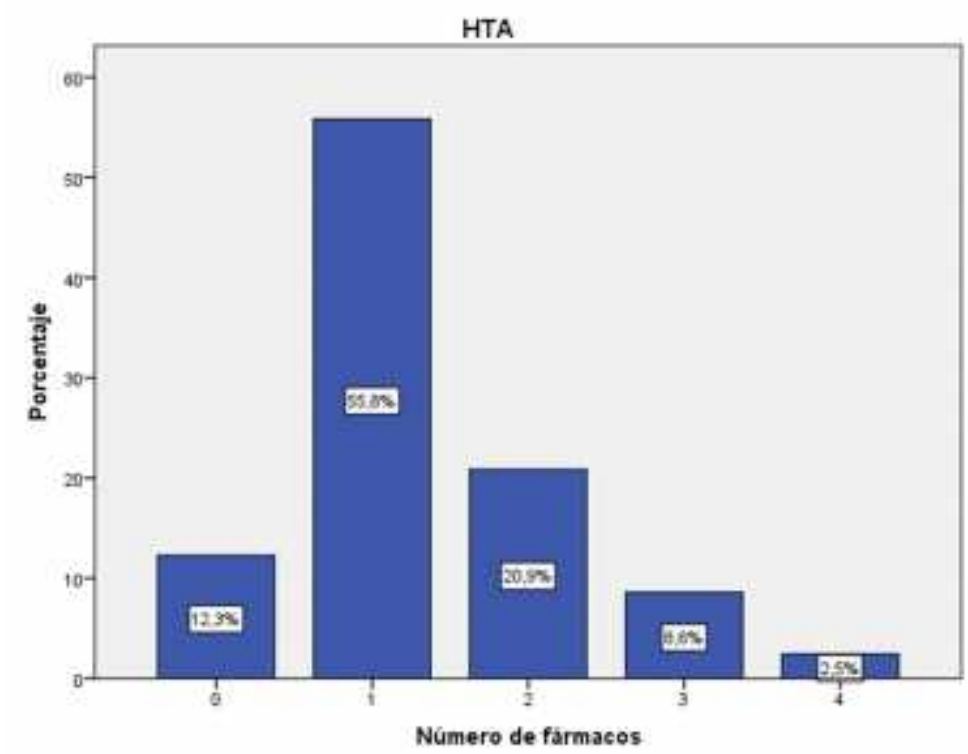

Figura 21. Número de fármacos para la HTA antes de la cirugía 
En los pacientes con diabetes mellitus: Ninguno 20 (23,5\%), Uno 39 (45,9\%), Dos $20(23,5 \%)$, Tres $6(7,1 \%)$. Mediana: 1 . El total de pacientes en los que se recoge este dato es 85 , todos los pacientes diabéticos.

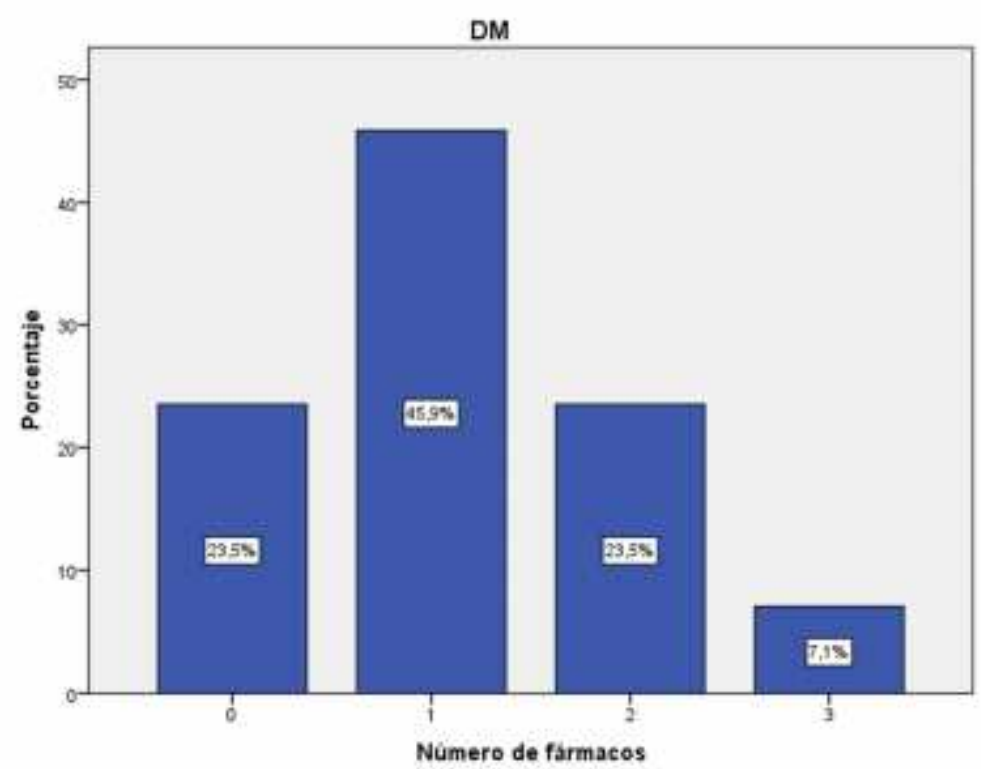

Figura 22. Número de fármacos para la DM antes de la cirugía

En la dislipemia: Ninguno 38 (52,1\%), Uno $32(43,8 \%)$, Dos $3(4,1 \%)$. Mediana: 1. El total de pacientes en los que se recoge este dato es 73 , un $97,3 \%$ de los que padecen dislipemia.

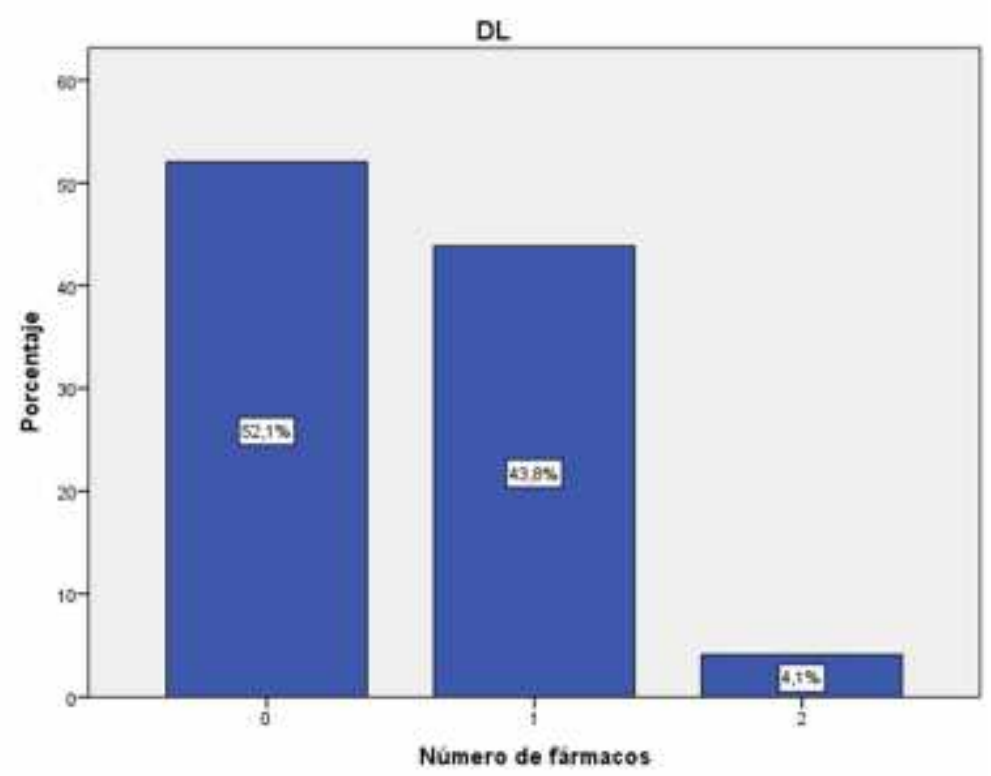

Figura 23. Número de fármacos para la $\mathrm{DL}$ antes de la cirugía 
Los pacientes con artrosis: Ninguno 19 (22,9\%), Uno 48 (57,8\%), Dos 11 $(13,3 \%)$, Tres $4(4,8 \%)$, Cuatro $1(1,2 \%)$. Mediana: 1 . El total de pacientes en los que se recoge este dato es 83 , un $77,5 \%$ de los que padecen artrosis.

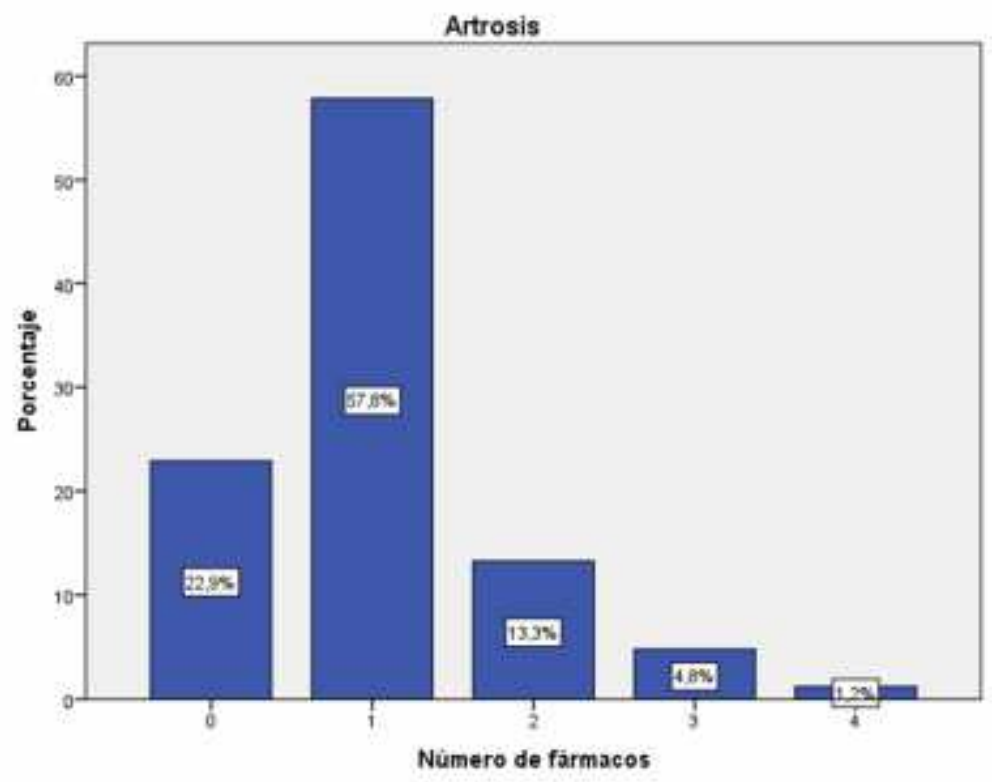

Figura 24. Número de fármacos para la artrosis antes de la cirugía

En aquellos con depresión: Ninguno 23 (19\%), Uno 47 (38,8\%), Dos 38 $(31,4 \%)$, Tres $11(9,1 \%)$, Cuatro $2(1,7 \%)$. Mediana: 1. El total de pacientes en los que se recoge este dato es 121 , un $97,5 \%$ de los que padecen depresión.

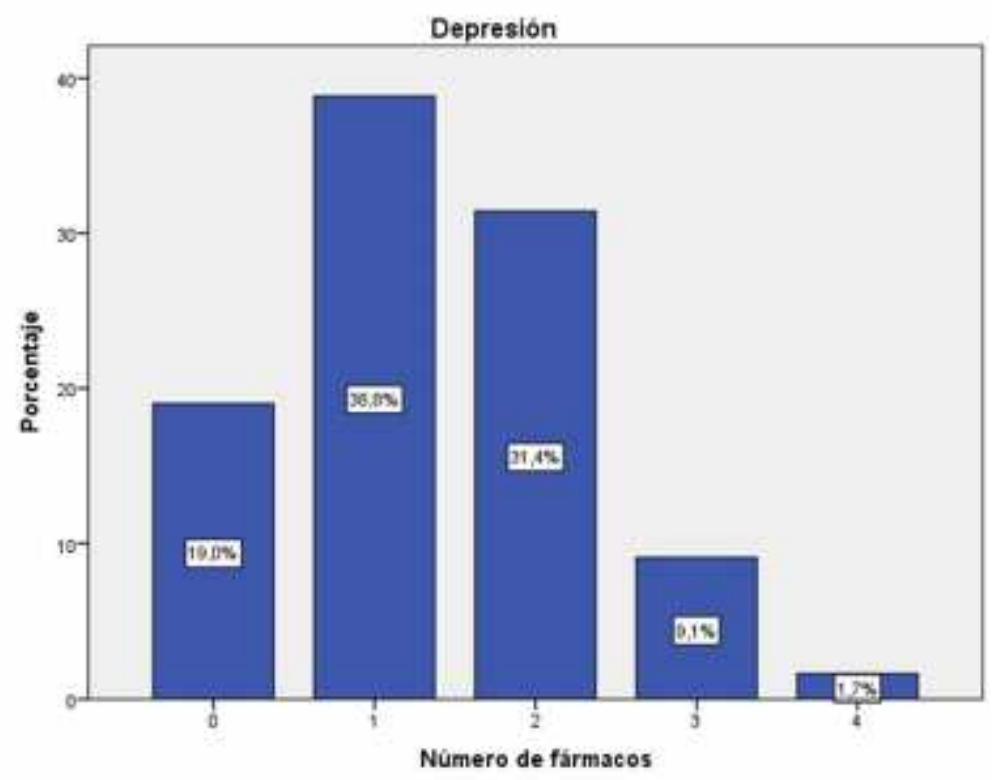

Figura 25. Número de fármacos para la depresión antes de la cirugía 
De los 178 pacientes con SAHS usaban CPAP 174 pacientes $(97,8 \%)$.

No tomaban ningún tipo de fármaco 77 pacientes de la serie (25\%), de éstos 34 tenían SAHOS y 31 usaban la CPAP. En definitiva, en 46/307 pacientes (15\%) no se cuantificó gasto terapéutico, bien por no tenerlo o por escaparse a la recogida de datos, como sucedió en un caso de los cuatro que padecen dislipemia de este grupo, y en tres de los cinco que padecen artrosis, las patologías que presentan peor recogida de fármacos.

El número total de fármacos consumidos para las patologías mencionadas es de 602, para un total de 210 pacientes que tomaba al menos un fármaco. Una relación de 2,86 fármacos/paciente medicado.

\section{Estudios preoperatorios}

Los resultados de la evaluación endocrinológica y psiquiátrica deben ser observados con precaución. Como todos los pacientes son valorados por Endocrinología, previo a la remisión a nuestra Unidad una vez evaluados y compensados, no debe extrañar que sólo $2(0,7 \%)$ tuvieran algún problema por mal control de la DM, o que en la evaluación psiquiátrica sólo 9 de ellos (3\%) presentaran algún tipo de problema, generalmente de tipo alimenticio, que precisaron de un seguimiento previo a la cirugía.

Se realizó gastroscopia preoperatoria a 304 pacientes (99\%), fue normal y sin Helicobacter pylori en 98 pacientes $(32,2 \%)$, el resto lo más frecuente fue la presencia de Helicobacter pylori en 158 pacientes $(51,9 \%)$, que en 22 casos se asociaba a gastritis o úlcera $(7,2 \%)$. De los que faltan, 29 pacientes tenían gastritis $(9,5 \%), 12$ esofagitis $(3,9 \%)$ y $7(2,3 \%)$ presentaban otra patología, generalmente pólipos hiperplásicos. De los 3 a los que no se realizó gastroscopia sabemos que eran Helicobacter pylori positivo. Se consiguió erradicar el Helicobacter en 139 enfermos (86,3\%).

Siguiendo el protocolo establecido la mayoría de los pacientes fueron sometidos a una ecografía preoperatoria, 306 (99,6\%). Fue normal en 131 $(42,8 \%), 117$ presentaban esteatosis $(38,2 \%), 34$ colelitiasis $(11,1 \%)$ y 8 ambas $(2,6 \%)$. 
El estudio del sueño se realizó a $278(90,5 \%)$ pacientes, fue normal en 100 $(35,9 \%)$ y $178(64 \%)$ presentaban SAHS.

El ecocardiograma se realizó a 220 pacientes (71,6\%), fue normal en 200 $(90,9 \%)$.

\section{Lista de espera quirúrgica}

El tiempo de espera para la intervención desde que el paciente es valorado en nuestra consulta y con todos los estudios correctos para incluirlo en la lista de espera, hasta el día de la intervención fue de 12,2 $(3,8)$ meses, mediana de 12, rango de 1 a 27,8 meses.

\subsection{Pacientes con BIG}

La colocación de un BIG previo a la cirugía se indicó en 46/307 pacientes (15\%). La media de pérdida de peso con respecto al día previo a su colocación, fue de $24,6(9,9) \mathrm{kg}$.

El tiempo consumido del área quirúrgica fue de $53,2(20,2)$ minutos, con un rango de 25,2-130,2 minutos.

Presentaron complicación con el BIG 7/46 pacientes (15,6\%), la más frecuente los vómitos, y una neumonía en un paciente. Fue necesaria la retirada anticipada en 2/46 pacientes, uno a la semana de la colocación, y otro al mes y medio.

La media de tiempo de aplicación de BIG estuvo en 4,2 (0,5) meses.

Realizamos el cálculo de los pacientes que tuvieron un BIG previo, con las tres medidas ponderales mensuales y la previa a la cirugía. La evolución ponderal se describe en la tabla siguiente. 
Tabla 14. Evolución ponderal durante el periodo con BIG, media (DE) / rango

\begin{tabular}{|l|c|c|c|c|c|c|}
\hline & $\begin{array}{l}\text { Primera } \\
\text { consulta }\end{array}$ & Pre-BIG & Revisión 1 & Revisión 2 & Revisión 3 & Pre-cirugía \\
\hline Peso (kg) & $157(23,2)$ & $160,2(23,5)$ & $145,3(19,7)$ & $142,9(21,2)$ & $138,8(22)$ & $136,3(22,1)$ \\
& $120-217$ & $119-222$ & $112-214$ & $112-207$ & $103-207$ & $97-207$ \\
\hline IMC & $60,1(5)$ & $61,4(5,5)$ & $56,2(5,5)$ & $54,8(5,6)$ & $53,2(5,9)$ & $52,2(5,6)$ \\
$\left(\mathrm{kg} / \mathrm{m}^{2}\right)$ & $50,8-71,2$ & $48,2-75,3$ & $45,4-69$ & $44,4-67,4$ & $40,6-67,8$ & $40,2-64,5$ \\
\hline Diferen. de & - & - & $14,1(6,8)$ & $18(7,8)$ & $22,1(9,6)$ & $24,6(9,9)$ \\
peso (kg) & & & $3-37$ & $4-42$ & $2-53$ & $3-54$ \\
\hline PSP (\%) & - & - & $14,1(6,4)$ & $17,9(7,5)$ & $22,1(9,5)$ & $24,7(9,9)$ \\
& & & $2,7-35,5$ & $5,2-41,3$ & $3,2-52,1$ & $4,8-53,1$ \\
\hline PEIMCP & - & - & $14,8(6,6)$ & $18,8(7,7)$ & $23,1(9,8)$ & $25,7(10,3)$ \\
$(\%)$ & & & $2,8-36,6$ & $5,4-43$ & $3,4-54,3$ & $5,2-55,3$ \\
\hline Pacientes & 46 & 46 & 45 & 44 & 44 & 44 \\
\hline
\end{tabular}

IMC: Índice de masa corporal. PSP: Porcentaje de sobrepeso perdido. PEIMCP: Porcentaje de exceso de IMC perdido. BIG: Balón intragástrico.

\subsection{Estudio descriptivo de cirugía}

Se realizó un BPG a todos los pacientes. De acuerdo a lo establecido, varió la longitud de las asas en función del IMC, fue corto en $79 / 307$ pacientes $(25,7 \%)$, largo en $164 / 307(53,4 \%)$ y distal en $64 / 307$ (20,8\%). El abordaje inicial fue abierto en 189/307 (61,5\%) casos a los que hay que sumar 9 conversiones de la cirugía laparoscópica a abierta, quedan por tanto 198/307 (64,5\%). Se programó vía laparoscópica en 118/307 (38,4\%), sin embargo hubo 9 conversiones 9/118 (7,6\%), quedando 109/307 (35,5\%) laparoscópicos. En la tabla 15 se recogen las características de la serie en función del abordaje. 
Tabla 15. Características de la población en función del abordaje quirúrgico

\begin{tabular}{|l|c|c|c|}
\hline Variable (n=307) & Abierto (n=198) & Laparoscópico (n=109) & p \\
\hline Edad, media (DE), años & $43,9(10)$ & $42,7(10)$ & 0,333 \\
\hline Sexo (Hombre/Mujer) (\%) & $21,2 / 78,8$ & $23,9 / 76,1$ & 0,594 \\
\hline Talla, media (DE), cm & $162(0,08)$ & $162,9(0,08)$ & 0,370 \\
\hline Peso, media (DE), kg & $137,7(22,1)$ & $120,9(14,7)$ & $<0,001$ \\
\hline IMC, media (DE), kg/m² & $52,3(6,6)$ & $45,5(4,4)$ & $<0,001$ \\
\hline BPG corto (\%). N=79 & $21(26,6)$ & $58(73,4)$ & $<0,001$ \\
\hline BPG largo (\%). N=164 & $114(69,5)$ & $50(30,4)$ & $<0,001$ \\
\hline BPG distal (\%). N=64 & $63(98,4)$ & $1(1,6)$ & $<0,001$ \\
\hline $\begin{array}{l}\text { Tiempo quirúrgico, media } \\
\text { (DE), minutos }\end{array}$ & $265,2(45,9)$ & $328,4(47,9)$ & $<0,001$ \\
\hline $\begin{array}{l}\text { Complicaciones que demoran } \\
\text { el alta (\%) }\end{array}$ & $28(14,1)$ & & 0,726 \\
\hline Estancia, media (DE), días & $13(29,7)$ & $13(11,9)$ & \\
\hline PSP a los 2 años & $67,53(18,36)$ & $72,92(18,12)$ & 0,018 \\
\hline
\end{tabular}

DE: Desviación estándar. IMC: Índice de masa corporal. BPG: Bypass gástrico. PSP: Porcentaje de sobrepeso perdido.

En 117 pacientes de toda la serie $(38,1 \%)$, se asoció alguna técnica quirúrgica, la más frecuente fue la colecistectomía en 97 casos (31,6\%), seguida de 12 hernioplastias, 12 eventroplastias $(3,9 \%)$ y otras en menor número: resección de divertículo de Meckel en 4 pacientes, herniorrafia también en 4 , apendicectomía y gastrectomía en 2 y una hemicolectomía derecha.

El tiempo quirúrgico, entendido como tiempo de utilización del área quirúrgica, tuvo una media de $287,7(55,5)$ minutos y un rango de 178-505; este tiempo está recogido para todos los pacientes. Si nos referimos al tiempo invertido por el cirujano, la media fue de $158,5(44,4)$ minutos con un rango de $80-320$; este tiempo depende de que se refleje en el protocolo del alta y está recogido de 243 pacientes.

La estancia hospitalaria media fue de 11,2 (24,1) días, con un rango de 5-407.

Presentaron algún tipo de complicación que demoró el alta 41 pacientes de las serie $(13,4 \%)$. El total de pacientes con complicación, independiente de si 
demoró o no el alta, fue de 99 (32,2\%) entre sistémicas y quirúrgicas. 66 $(21,4 \%)$ tuvieron sólo complicaciones quirúrgicas, 22 (7,1\%) una complicación médica y $11(3,5 \%)$ ambas. En cinco enfermos hubo más de una complicación quirúrgica. Las complicaciones se detallan en la tabla 16.

Tabla 16. Complicaciones postoperatorias. $99 / 307$ casos $(32,2 \%)$

\begin{tabular}{|l|l|c|c|c|}
\hline & Tipo & Número & \% Total & \% Ajustado \\
\hline Sistémica (33) & & & & \\
\hline & ITU & 19 & 6,1 & 57,5 \\
\hline & Neumonía & 4 & 1,3 & 12,1 \\
\hline Quirúrgica (84) & Otras & 10 & 3,2 & 30,3 \\
\hline & IHQ & & & \\
\hline & Hemorragia & 9 & 2,9 & 10,7 \\
\hline & Dehiscencia & 8 & 2,6 & 9,5 \\
\hline & Obstrucción & 4 & 1,3 & 4,7 \\
\hline & Absceso & 4 & 1,3 & 4,7 \\
\hline & Peritonitis & 3 & 1 & 3,5 \\
\hline & Otras & 10 & 3,2 & 11,9 \\
\hline & & & &
\end{tabular}

ITU: Infección del tracto urinario. IHQ: Infección de herida quirúrgica.

Las complicaciones sistémicas si las diferenciamos por el tipo de abordaje, presentaron ITU 14/189 pacientes de cirugía abierta (7,4\%), y 5/109 (4,5\%) de cirugía laparoscópica. La neumonía en 3/189 sometidos a laparotomía (1,5\%) y $1 / 109$ a laparoscopia $(0,9 \%)$.

En las complicaciones quirúrgicas destaca la infección de la herida operatoria, $33 / 189(17,4 \%)$ en cirugía abierta, $10 / 109(9,1 \%)$ en los pacientes de cirugía laparoscópica, y 3/9 (33,3\%) en los que precisaron conversión.

La hemorragia fue más frecuente en el grupo laparoscópico 4/109 (3,6\%) que en cirugía abierta 4/189 (2\%), el noveno era del grupo de conversión. La dehiscencia de sutura siguió un patrón inverso a la hemorragia: 2/109 (1,8\%) en los sometidos a laparoscopia, 5/189 (2,6\%) casos en cirugía abierta, el 
octavo era del grupo de conversión. Los cuatro casos con obstrucción intestinal fue en el grupo de abordaje laparoscópico, en uno de ellos fue necesario convertir. El absceso abdominal fue diagnosticado en 3/189 pacientes de cirugía abierta (1,5\%), y 1/109 laparoscópica $(0,9 \%)$.

Como queda recogido en la tabla 17 precisaron reintervención 14/307 (4,5\%) de los pacientes sometidos a BPG o $14 / 77(18,1 \%)$ de los pacientes que presentaron complicación quirúrgica. Las 4 dehiscencias de sutura reintervenidas, eran fugas de la anastomosis gastro-yeyunal, una de ellas condicionada por obstrucción del pie de asa; se realizó sutura y colocación de gastrostomía de alimentación en tres. Dos obstrucciones del pie de asa y una tercera por hernia incarcerada en un orificio de trócar, todas en cirugía laparoscópica. Una hemorragia de la línea de sección del estómago excluido y una colecistitis alitiásica. Perforación inadvertida de intestino delgado durante la laparoscopia 3.Las dos restantes fueron drenajes de abscesos, uno de ellos un empiema izquierdo tras abordaje laparoscópico.

La mayoría de estas reintervenciones tuvieron lugar en el postoperatorio inmediato y sin haber causado alta tras la intervención inicial. Sin embrago dos de ellas fueron con reingreso dentro de los treinta días posteriores al alta.

Según el tipo de abordaje inicial, 3/189 fueron en cirugía abierta (1,5\%), 8/109 en laparoscópica $(7,3 \%)$, y 3/9 en conversión (33,3\%).

Tabla 17. Causas de reintervención según abordaje del BPG. 14/307 pacientes

\begin{tabular}{|l|c|c|c|}
\hline & Abierto $\mathbf{n = 1 8 9}$ & Laparoscópico $\mathbf{n = 1 0 9}$ & Conversión $\mathbf{n = 9}$ \\
\hline Dehiscencia & 2 & 1 & 1 \\
\hline Obstrucción & - & 3 & - \\
\hline Hemorragia & - & - & - \\
\hline $\begin{array}{l}\text { Perforación de } \\
\text { intestino delgado }\end{array}$ & - & 3 & - \\
\hline Absceso & 1 & 1 & 1 \\
\hline Colecistitis & - & - & - \\
\hline
\end{tabular}


La medicación al alta para todos los pacientes consistió en: Clexane 40mg una inyección subcutánea al día durante un mes (135€/30 días), y Omeprazol 20mg (2,4€/30 días) hasta acudir a la consulta, aproximadamente al mes de la cirugía, suplemento multivitamínico $(0,125 € /$ día $)$ y de hierro, generalmente Tardyferon 80mg 1 comprimido al día (0,08€/día).

\subsection{Seguimiento de la intervención}

Los resultados de la cirugía los analizamos en las tres categorías más destacables o las que persigue este tipo de tratamiento: el peso, las comorbilidades y la calidad de vida.

\subsubsection{Evolución ponderal}

La evolución ponderal toma como referencia inicial el peso en la primera consulta (peso consulta), seguida del peso el día previo a la intervención (peso inicial), que en el caso de haber tenido un BIG previo además contaremos con varias mediciones entre ambos momentos. Los siguientes registros serán postoperatorios, revisión 1 (al mes), revisión 2 (6 meses), revisión 3 (año), revisión 4 (dos años). Calculamos la pérdida de peso, el PSP y el PEIMCP.

Tabla 18. Evolución ponderal de toda la serie, media (DE) / rango

\begin{tabular}{|l|c|c|c|c|c|c|}
\hline & $1^{\text {a }}$ consulta & Peso inicial & Revisión 1 & Revisión 2 & Revisión 3 & Revisión 4 \\
\hline Peso $(\mathrm{kg})$ & $131,8(21,4)$ & $127,3(17,8)$ & $111,1(15,6)$ & $89,4(14,3)$ & $81,8(14,2)$ & $81,8(14,9)$ \\
& $95-219$ & $89-207$ & $77-168$ & $59-138,5$ & $50-145$ & $49-150$ \\
\hline IMC & $49,9(6,8)$ & $48,2(5,3)$ & $42,1(5)$ & $33,9(4,8)$ & $31,1(4,9)$ & $31,1(5,2)$ \\
$\left(\mathrm{kg} / \mathrm{m}^{2}\right)$ & $36,2-77,6$ & $35-64,5$ & $22,6-59,1$ & $21,7-53,2$ & $20,8-47,4$ & $20,1-48,9$ \\
\hline PSP (\%) & - & - & $25(7,9)$ & $58,6(13,6)$ & $69,8(18,4)$ & $69,3(18,4)$ \\
& & & $4,8-59,6$ & $24,3-93,5$ & $18,8-107,6$ & $13,3-119,5$ \\
\hline PEIMCP & - & - & $26,9(8,7)$ & $63,3(15,5)$ & $75,1(18,3)$ & $74,7(20,2)$ \\
$(\%)$ & & & $5,2-62,6$ & $26-132,1$ & $19,9-116,7$ & $14-134,6$ \\
\hline Pacientes & 307 & 307 & 305 & 294 & 288 & 287 \\
\hline
\end{tabular}

IMC: Índice de masa corporal. PSP: Porcentaje de sobrepeso perdido. PEIMCP: Porcentaje de exceso de IMC perdido. 


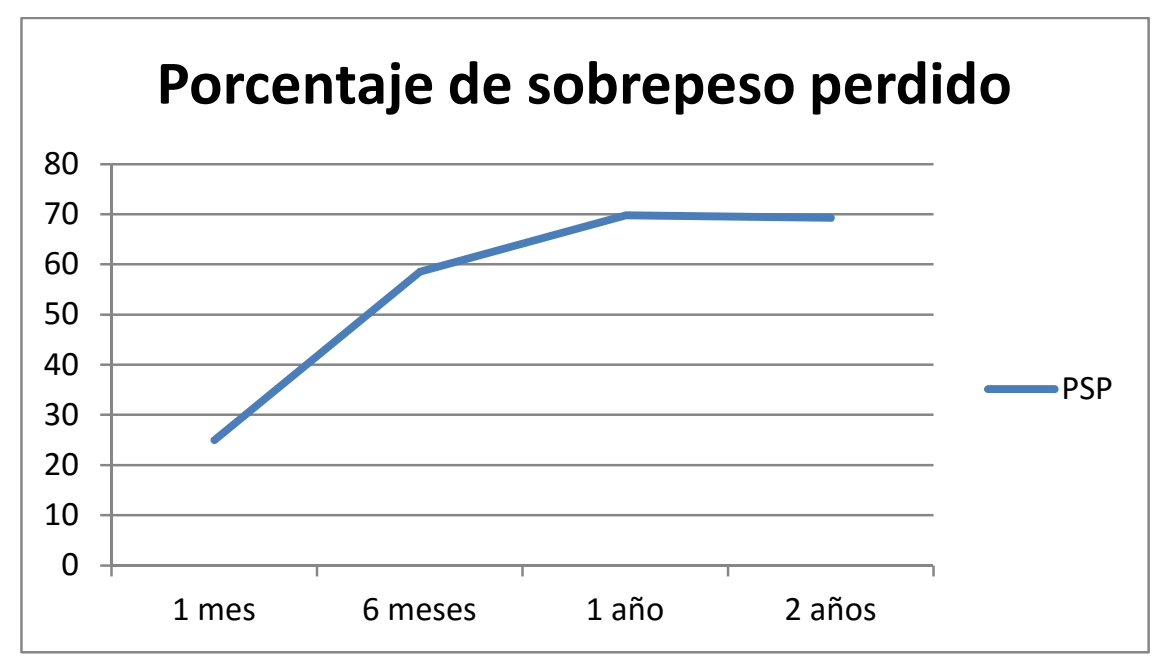

Figura 26. Evolución ponderal por porcentaje de sobrepeso perdido

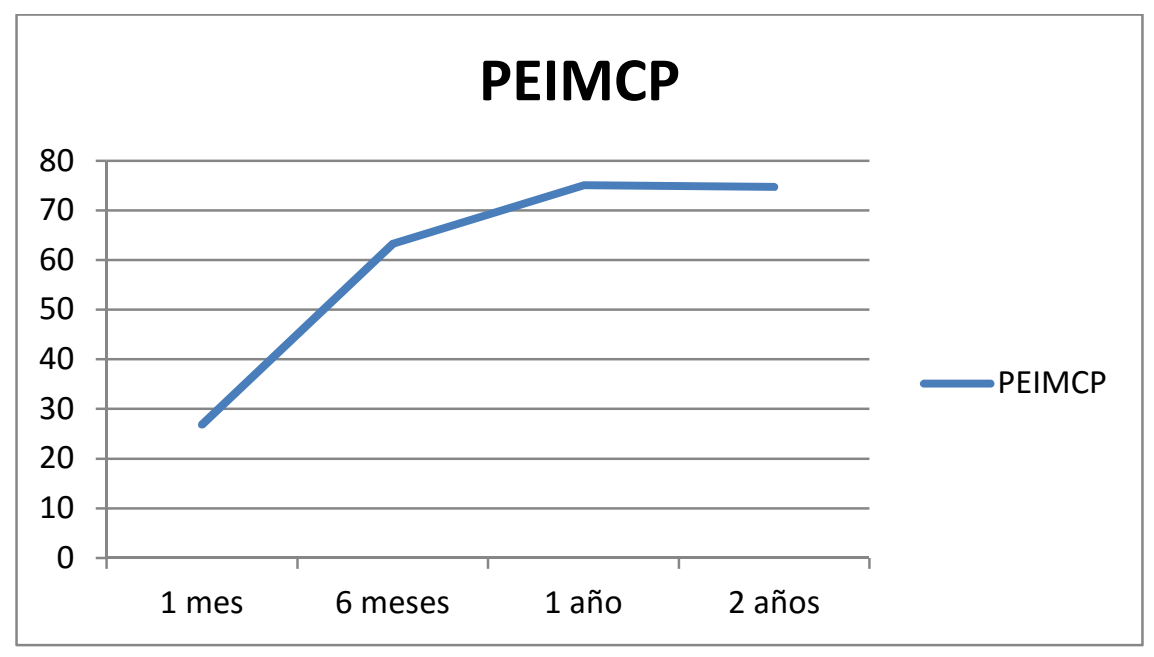

Figura 27. Evolución ponderal por porcentaje de exceso de IMC perdido 
Si comparamos en función del abordaje, abierto o laparoscópico, la evolución queda recogida en la figura siguiente.

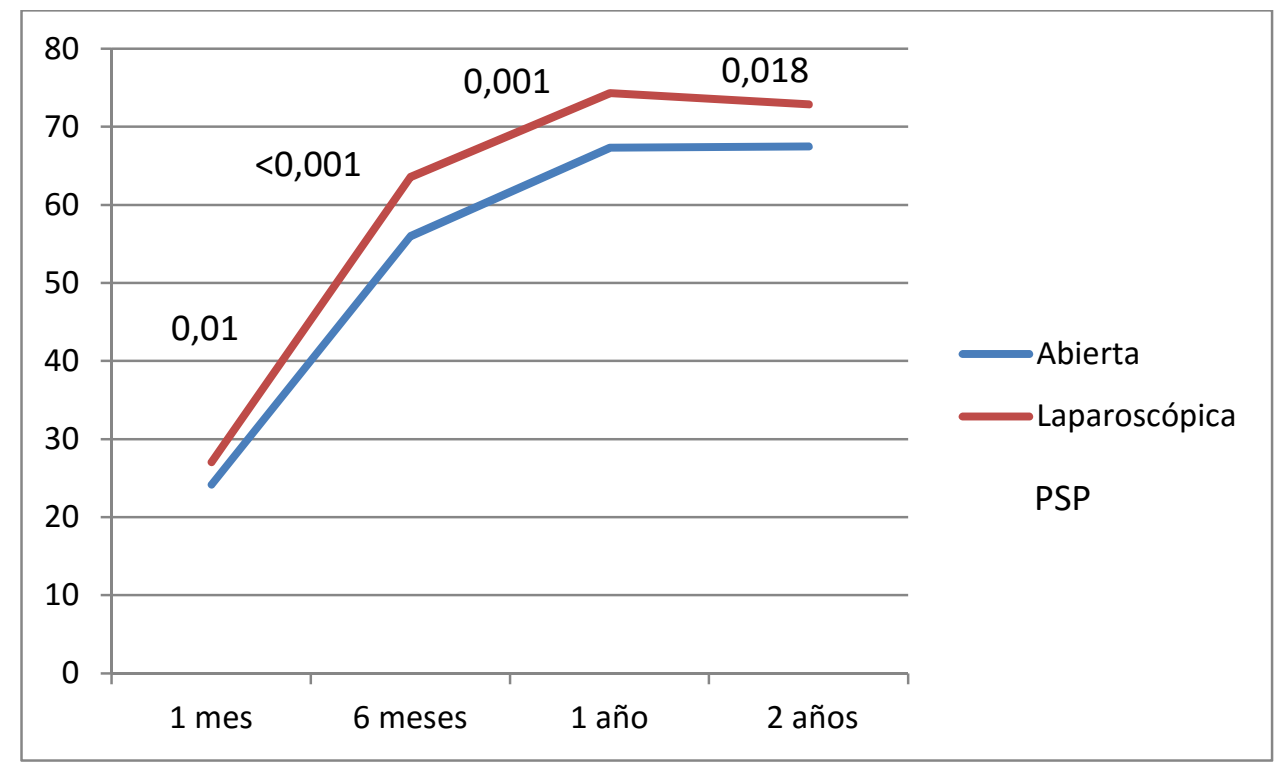

Figura 28. Porcentaje de sobrepeso perdido en el seguimiento, según abordaje

Si comparamos aquellos que tuvieron un BIG previo, la diferencia de PSP es la recogida en la figura 29.

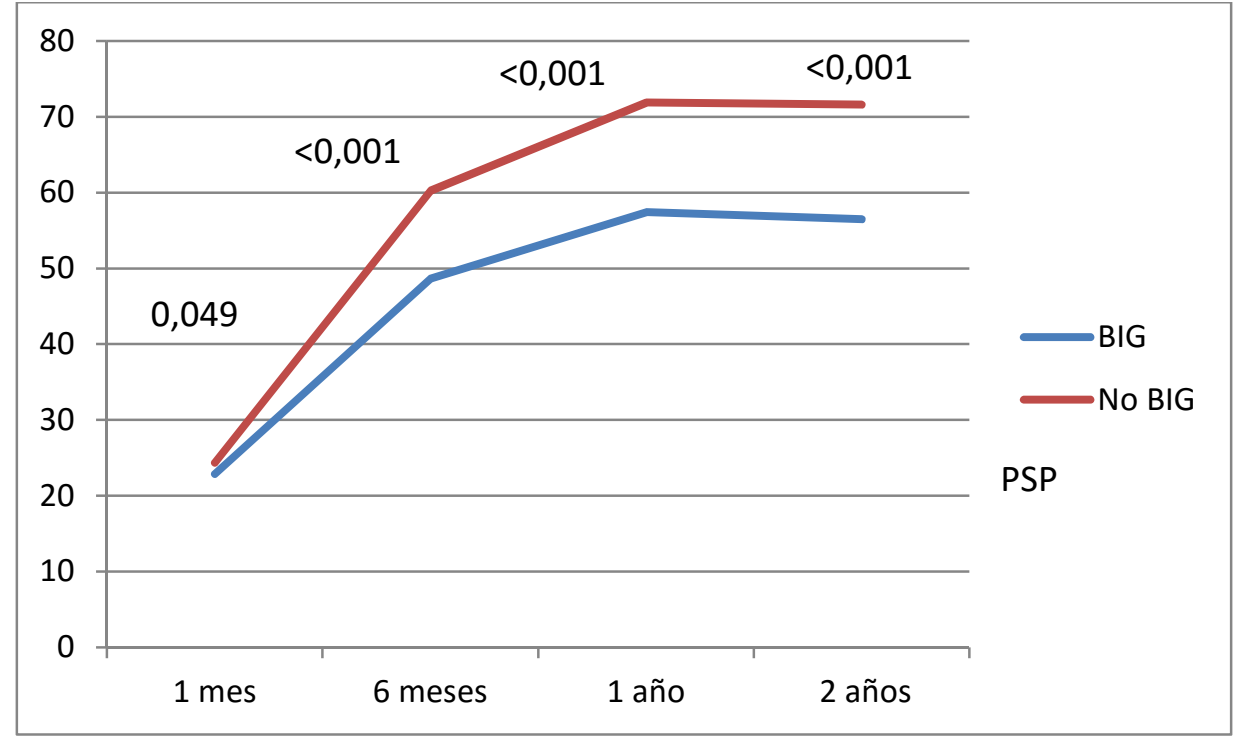

Figura 29. Porcentaje de sobrepeso perdido en el seguimiento, según BIG previo 


\subsubsection{Modificación de fármacos tras la cirugía por patologías}

\section{HTA}

Partimos de 164/307 pacientes con HTA (53,4\%), de los que 143 de los 163 recogidos (88\%) tomaban al menos un fármaco y 20 pacientes no están medicados. La media de fármacos antes de la cirugía era $1,33(0,88)$. Su evolución en las sucesivas revisiones viene recogida en la tabla y figura siguientes:

Tabla 19. Evolución postoperatoria de la HTA

\begin{tabular}{|l|c|c|c|c|}
\hline & R1 (mes) & R2 (6 meses) & R3 (año) & R4 (2 años) \\
\hline Igual (\%) & $45(27,8)$ & $7(4,4)$ & $5(3,2)$ & $5(3,2)$ \\
\hline Mejor (\%) & $53(32,7)$ & $55(34,8)$ & $41(26,3)$ & $33(21,3)$ \\
\hline Resuelta (\%) & $64(39,5)$ & $96(60,8)$ & $110(70,5)$ & $117(75,5)$ \\
\hline Media de fcos (DE) & $0,74(0,84)$ & $0,46(0,68)$ & $0,37(0,65)$ & $0,3(0,61)$ \\
\hline Pacientes (n) & 162 & 158 & 156 & 155 \\
\hline
\end{tabular}

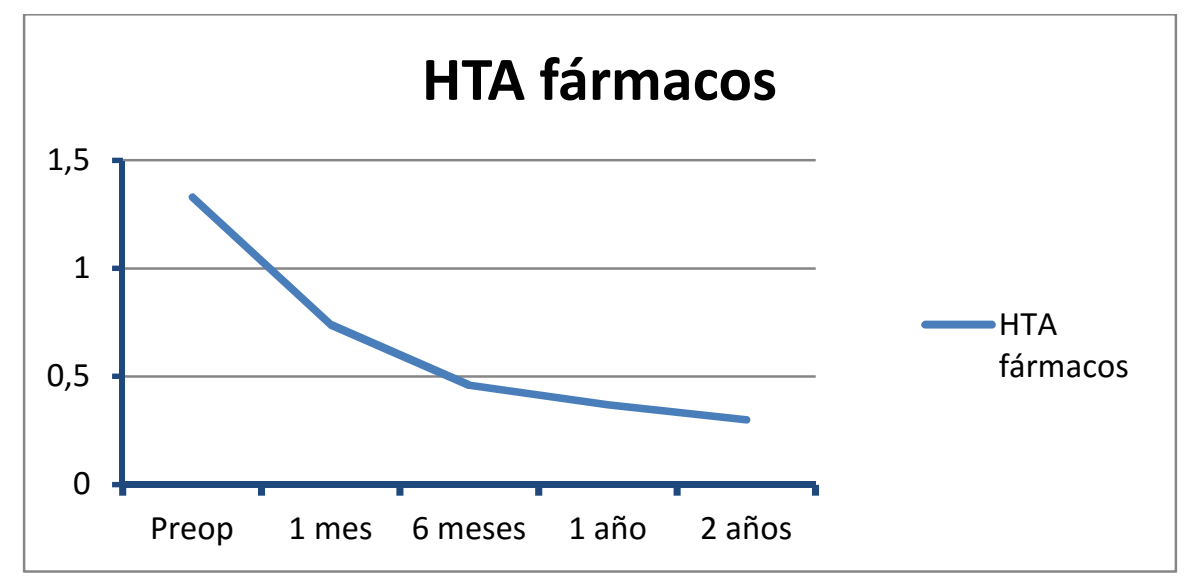

Figura 30. Número de fármacos para la HTA en el seguimiento 


\section{DM}

Partimos de 85 pacientes con DM (27,7\%), de los que 65 (76,5\%) recibían al menos un fármaco y 20 no estaban medicados. La media de fármacos/paciente antes de la cirugía era $1,14(0,86)$. La evolución en las sucesivas revisiones viene recogida en la tabla y figura siguientes:

Tabla 20. Evolución postoperatoria de la DM

\begin{tabular}{|l|c|c|c|c|}
\hline & R1 (mes) & R2 (6 meses) & R3 (año) & R4 (2 años) \\
\hline Igual (\%) & $6(7,1)$ & $1(1,2)$ & - & - \\
\hline Mejor (\%) & $27(32,1)$ & $17(20,2)$ & $10(12)$ & $9(11)$ \\
\hline Resuelta (\%) & $51(60,7)$ & $66(78,6)$ & $73(88)$ & $73(89)$ \\
\hline Media de fcos (DE) & $0,37(0,53)$ & $0,2(0,4)$ & $0,11(0,31)$ & $0,11(0,35)$ \\
\hline Pacientes (n) & 84 & 84 & 83 & 82 \\
\hline
\end{tabular}

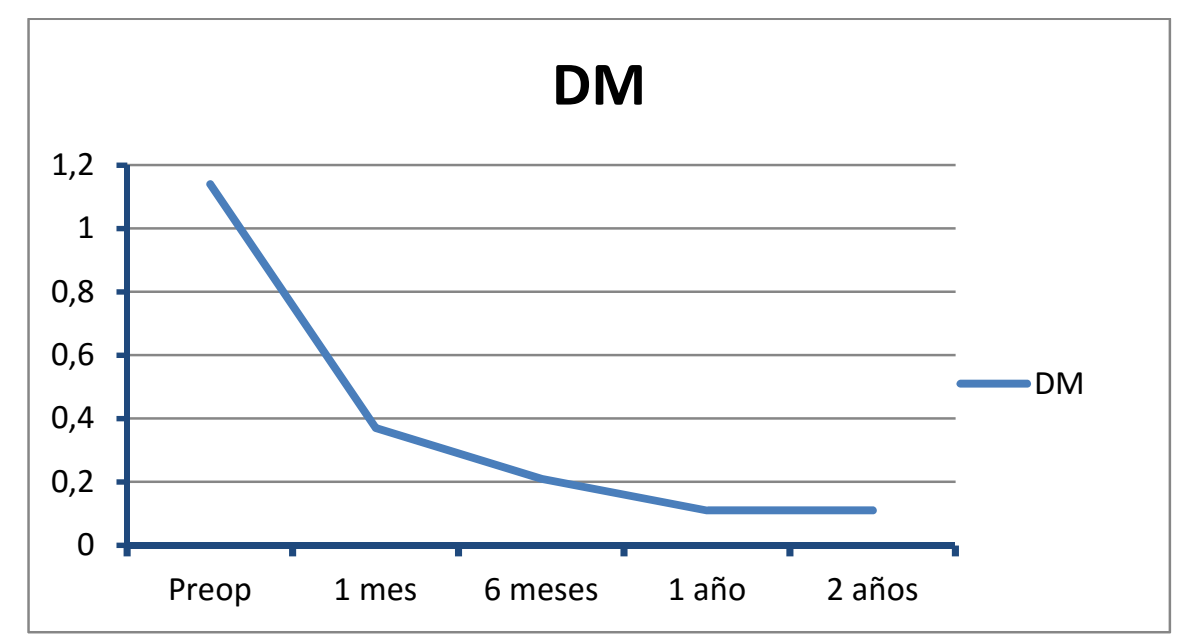

Figura 31. Número de fármacos para la DM en el seguimiento 


\section{DL}

De 73 pacientes con DL (23,7\%), 35 (48\%) tomaba al menos un fármaco y 38 no estaban medicados. La media de fármacos antes de la cirugía era 0,52 $(0,57)$. La evolución en las sucesivas revisiones viene recogida en la tabla y figura siguientes:

Tabla 21. Evolución postoperatoria de la DL

\begin{tabular}{|l|c|c|c|c|}
\hline & R1 (mes) & R2 (6 meses) & R3 (año) & R4 (2 años) \\
\hline Igual (\%) & $25(34,7)$ & $8(11,3)$ & $3(4,3)$ & $2(2,9)$ \\
\hline Mejor (\%) & $11(15,3)$ & $6(8,5)$ & $5(7,1)$ & $6(8,7)$ \\
\hline Resuelta (\%) & $36(50)$ & $57(80,2)$ & $62(88,6)$ & $61(88,4)$ \\
\hline Media de fcos (DE) & $0,28(0,51)$ & $0,17(0,41)$ & $0,07(0,26)$ & $0,07(0,26)$ \\
\hline Pacientes (n) & 72 & 71 & 70 & 69 \\
\hline
\end{tabular}

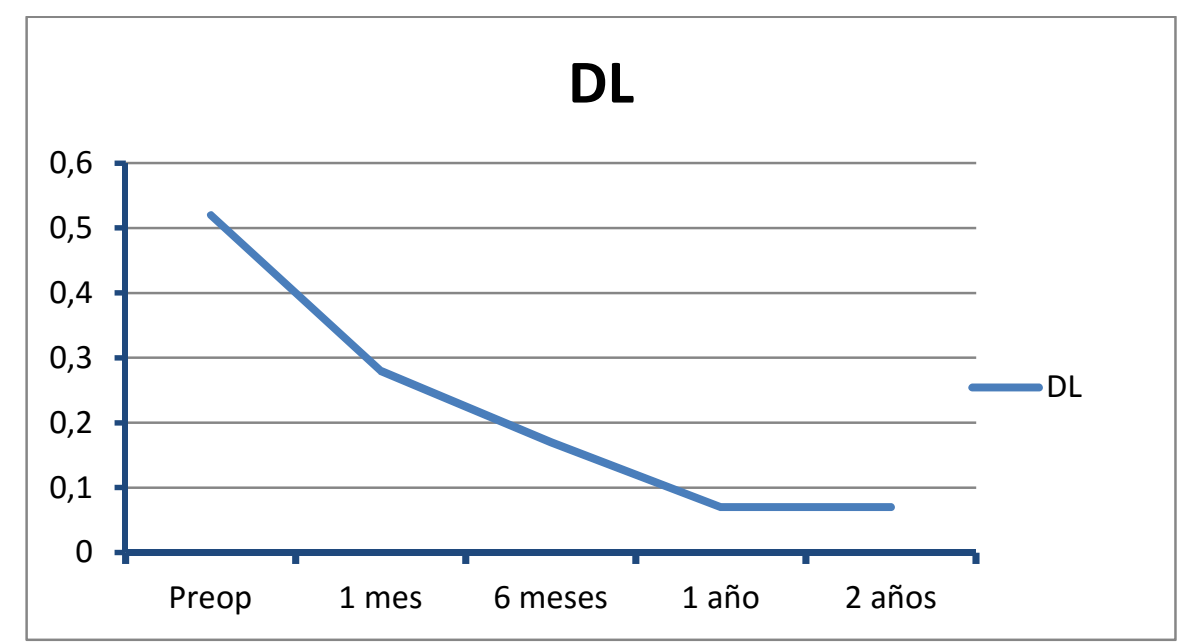

Figura 32. Número de fármacos para la DL en el seguimiento 


\section{SAHS}

De los 178 pacientes que padecían SAHS (58\%), 174 (98\%) usaban CPAP. La evolución en las sucesivas revisiones viene recogida en la tabla y figura siguientes:

Tabla 22. Evolución postoperatoria del SAHS

\begin{tabular}{|l|c|c|c|c|}
\hline & R1 (mes) & R2 (6 meses) & R3 (año) & R4 (2 años) \\
\hline Igual (\%) & $76(42,9)$ & $16(9,3)$ & $4(2,49$ & $4(2,4)$ \\
\hline Mejor (\%) & $53(29,9)$ & $47(27,3)$ & $29(17,4)$ & $13(7,8)$ \\
\hline Resuelta (\%) & $48(27,1)$ & $109(64,3)$ & $134(80,2)$ & $149(89,8)$ \\
\hline Uso de CPAP (\%) & 68 & 34 & 18 & 9 \\
\hline Pacientes (n) & 177 & 172 & 167 & 166 \\
\hline
\end{tabular}

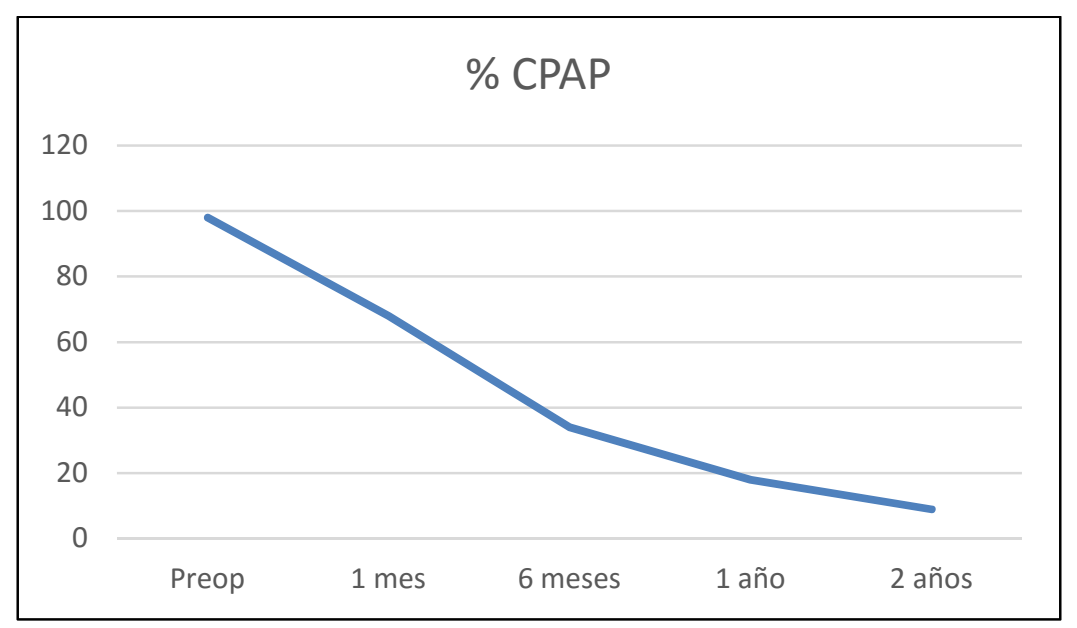

Figura 33. Porcentaje de pacientes que utilizan la CPAP en el seguimiento 


\section{Artrosis}

Ciento siete pacientes tenían artrosis (34,9\%), de los que teníamos recogida la medicación en 83, y 64/83 (77\%) tomaba al menos un fármaco. La media de fármacos antes de la cirugía era de 1,03 (0,81). La evolución en las sucesivas revisiones viene recogida en la tabla y figura siguientes:

Tabla 23. Evolución postoperatoria de la artrosis

\begin{tabular}{|l|c|c|c|c|}
\hline & R1 (mes) & R2 (6 meses) & R3 (año) & R4 (2 años) \\
\hline Igual (\%) & $53(73,6)$ & $18(25,7)$ & $10(14,5)$ & $8(11,8)$ \\
\hline Mejor (\%) & $19(26,4)$ & $49(70)$ & $42(60,9)$ & $38(55,9)$ \\
\hline Resuelta (\%) & - & $3(4,3)$ & $17(24,6)$ & $22(32,4)$ \\
\hline Media de fcos (DE) & $0,95(0,7)$ & $0,79(0,68)$ & $0,54(0,65)$ & $0,47(0,61)$ \\
\hline Pacientes (n) & 72 & 70 & 69 & 68 \\
\hline
\end{tabular}

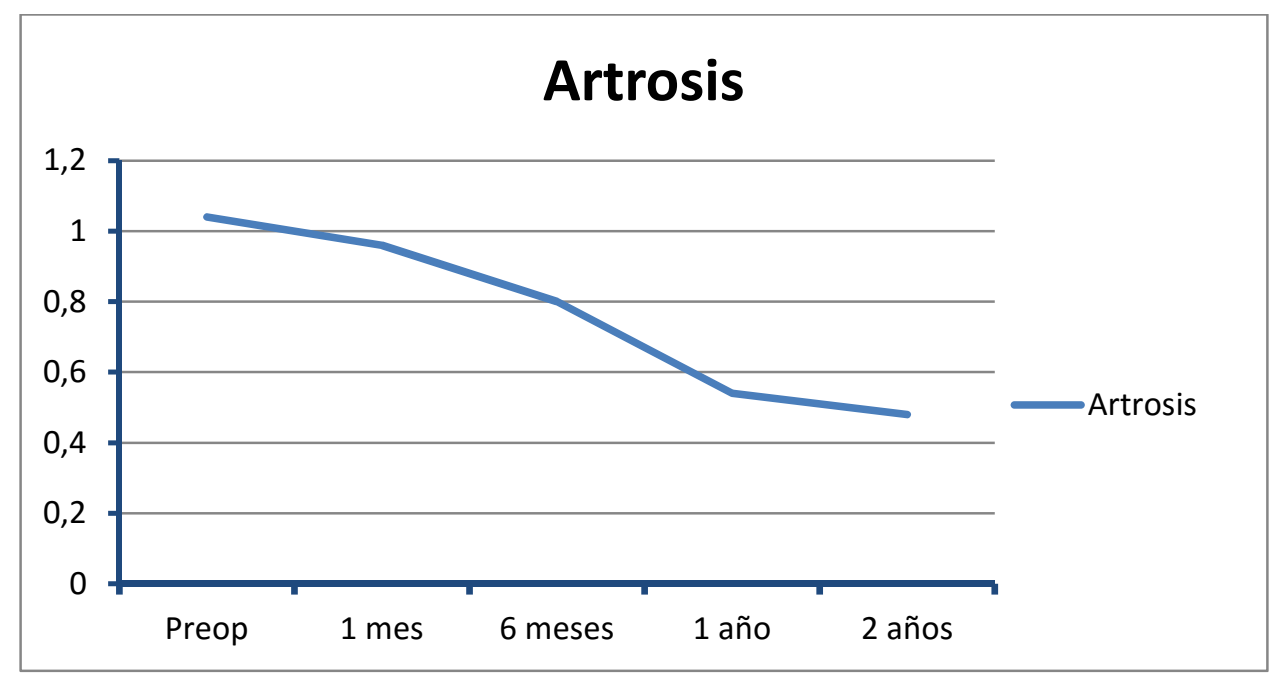

Figura 34. Número de fármacos para la artrosis en el seguimiento 


\section{Depresión}

En 123/307 pacientes se registró depresión (40\%), de los que 98 de los 121 recogidos $(81 \%)$ tomaba al menos un fármaco y 23 pacientes no estaban medicados. Su media de fármacos antes de la cirugía era de 1,35 $(0,94)$. La evolución en las sucesivas revisiones viene recogida en la tabla y figura siguientes:

Tabla 24. Evolución postoperatoria de la depresión

\begin{tabular}{|l|c|c|c|c|}
\hline & R1 (mes) & R2 (6 meses) & R3 (año) & R4 (2 años) \\
\hline Igual (\%) & $71(64)$ & $33(31,7)$ & $18(17,3)$ & $13(12,6)$ \\
\hline Mejor (\%) & $27(24,3)$ & $43(41,3)$ & $46(44,2)$ & $44(42,7)$ \\
\hline Resuelta (\%) & $13(11,7)$ & $28(26,9)$ & $40(38,5)$ & $46(44,7)$ \\
\hline Media de fcos (DE) & $1,14(0,94)$ & $0,92(0,89)$ & $0,72(0,84)$ & $0,7(0,83)$ \\
\hline Pacientes (n) & 111 & 104 & 104 & 103 \\
\hline
\end{tabular}

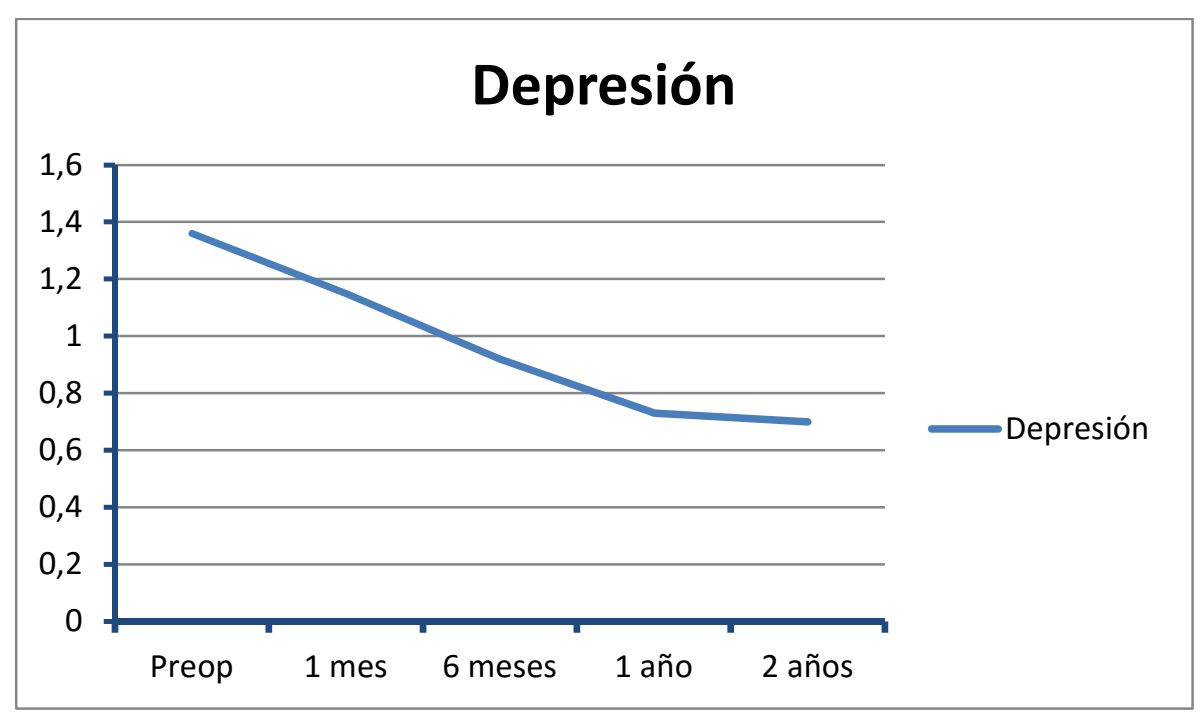

Figura 35. Número de fármacos para la depresión en el seguimiento 


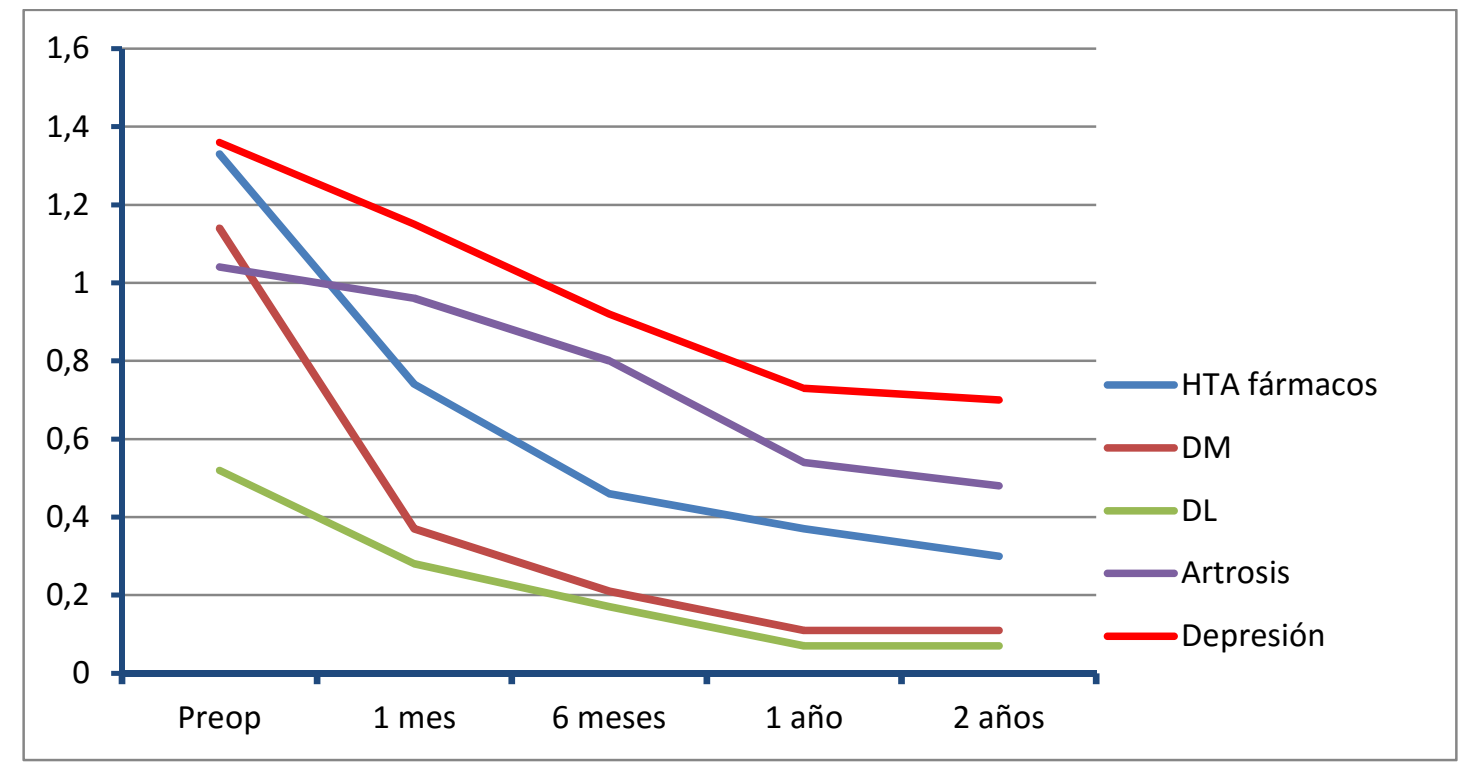

Figura 36. Número de fármacos para las principales comorbilidades, en el seguimiento

Evolución global de fármacos estudiados en la figura 36. La suma final de fármacos fue de 164, que de los 210 pacientes que estaban medicados al principio del estudio queda una relación de 0,78 fármacos por paciente, frente a los 2,86 antes de la intervención (602/210). Por grupos de peso, para la serie completa o para los que toman medicación, la evolución queda recogida en las tablas siguientes:

Tabla 25. Número de fármacos por paciente de la serie completa (307), por grupos de peso según su índice de masa corporal (IMC), en primera consulta

\begin{tabular}{|l|c|c|}
\hline & Antes de la cirugía & A los dos años \\
\hline IMC $<45$ & $167 / 76(2,19)$ & $43 / 76(0,56)$ \\
\hline IMC 45-55 & $332 / 169(1,96)$ & $99 / 169(0,58)$ \\
\hline IMC $>55$ & $103 / 62(1,66)$ & $22 / 62(0,35)$ \\
\hline
\end{tabular}

Tabla 26. Número de fármacos por paciente que toman fármacos (210), por grupos de peso según su índice de masa corporal (IMC), en primera consulta

\begin{tabular}{|l|c|c|}
\hline & Antes de la cirugía & A los dos años \\
\hline IMC $<45$ & $167 / 43(3,88)$ & $43 / 43(1)$ \\
\hline IMC 45-55 & $332 / 109(3,04)$ & $99 / 109(0,91)$ \\
\hline IMC $>55$ & $103 / 58(1,77)$ & $22 / 58(0,38)$ \\
\hline
\end{tabular}


La media de fármacos para la serie completa (307 pacientes), queda recogida en la figura siguiente.

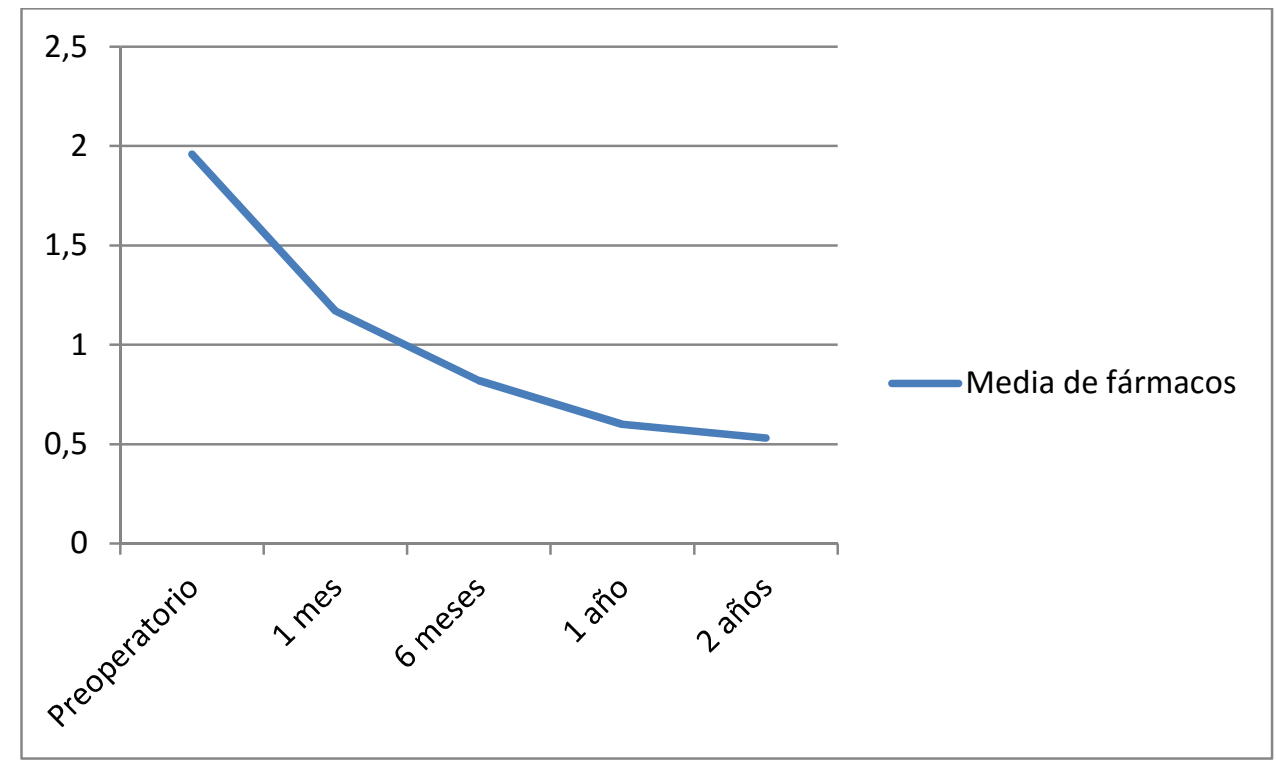

Figura 37. Evolución de la media de consumo de fármacos en el seguimiento

\subsubsection{Cálculo del ahorro en fármacos}

Calculamos el ahorro en fármacos que supone la resolución de las principales comorbilidades con las referencias ya explicadas:

- HTA: A los seis meses (lo consideramos como el momento de referencia para calcular el ahorro del primer año): Un 31,2\% de la muestra completa no tenía HTA. De acuerdo con el coste anual en fármacos por paciente con HTA de 894€, aplicado al global serían $279 €$ que dejamos de gastar. Si aceptamos una reducción de coste a la mitad de los que tienen mejoría en lugar de resolución, tenemos un $17,9 \%$ de la muestra, que transformado en coste de fármacos, serían $80 €$. Siendo el total al primer año 359€. Aplicado sólo a los que padecen HTA serían $699 €$. A los dos años un $40 \%$ de la muestra completa no presenta HTA, que aplicado al global podemos hablar de $357 €$ al año, sin tener en cuenta la 
progresión previa en disminución de gasto, ni el tiempo posterior en el que se mantendrá está resolución. Sumado al $21 \%$ de pacientes que ha disminuido la medicación, que representan el $10,7 \%$ de la muestra completa, y a mitad de coste, tendríamos $48 €$. El total al segundo año es

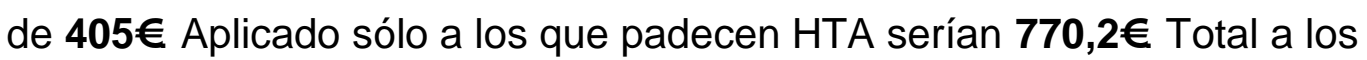
dos años: $764 €$ por paciente de la muestra y $1.469 €$ por paciente con HTA.

- DM: A los seis meses el $21,5 \%$ de la muestra completa ya no tenía DM, de un coste estimado en fármacos en nuestro país de $265 €$ al año, aplicado al global serían $57 €$. Si asumimos una reducción a la mitad de los que mejoran, tenemos un $5,5 \%$, que a mitad de coste serían $7 €$. Total 64€. Aplicado sólo a los que tienen DM serían 235€. A los dos años un $23,7 \%$ de la muestra se habrá curado, que en coste farmacéutico supone a $265 €$ al año, hablar de $63 €$ por paciente intervenido. Un 2,9\% presenta mejoría, al que corresponden $4 €$. Total: $67 €$. Aplicado sólo a los que tienen DM serían $250 €$. Total a los dos

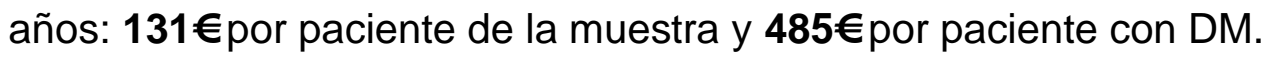

- DL: A los seis meses el $18,5 \%$ de la muestra completa ya no tenía DL, de un coste de $282 €$ al año, aplicado al global serían $52 €$. Si asumimos una reducción a la mitad de los que mejoran, tenemos un $2 \%$, que a mitad de coste serían $3 €$. Total $55 €$. Aplicado sólo a los que tienen DL serían $238 €$. A los dos años un 19,8\% de la muestra se habrá curado, que en coste farmacéutico supone a $282 €$ al año, hablar de $56 €$ por paciente intervenido. Un $2 \%$ presenta mejoría, al que corresponden $3 €$. Total: $59 €$. Aplicado sólo a los que tienen DL serían $262 €$. Total a los dos años: $114 €$ por paciente de la muestra y $500 €$ por paciente con $\mathrm{DL}$.

- SAHS: En este caso sólo nos fijamos en los que dejan de usar la CPAP. A los seis meses un $35,6 \%$ de la muestra ya no usarán la CPAP. El coste de la terapia CPAP es de unos $675 €$ anuales (1,85€ por sesión), lo que supone $240 €$ por paciente de la muestra. 445,5€ para los que tenían SAHS. A los dos años un $49,2 \%$ de la muestra no harán uso de la 
CPAP, lo que supone $332 €$ por paciente de la muestra y $614,2 €$ para los que tenían SAHS. Total a los dos años: $572 €$ por paciente de la muestra

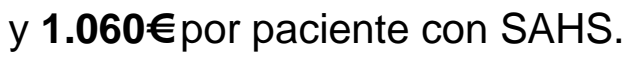

- Depresión: A los seis meses un 9,1\% de la muestra habrá dejado la medicación, la diferencia de coste entre pacientes que presentan remisión, respecto a los que no, es de $1.000 €$ anuales. En este caso supondrían $91 €$. No consideramos la mejoría. Para el grupo de paciente con depresión supondría un ahorro de 269€. A los dos años un $15 \%$ de la muestra habrá dejado la medicación, lo que representa $150 €$. Para el grupo de depresión sería de 447€. Total a los dos años: $241 €$ por paciente de la muestra y $716 €$ por paciente con depresión.

\subsubsection{Medicación profiláctica}

En este apartado se recoge la medicación que van a requerir los pacientes en suplementos vitamínicos y minerales, como consecuencia principalmente del componente malabsortivo de la cirugía. Los pacientes llevan por protocolo un suplemento multivitamínico tipo Supradyn $\AA$ un comprimido al día $(0,125 € /$ día $)$ y hierro oral tipo Tardyferon un comprimido al día $(0,08 € /$ día). La evolución del tratamiento profiláctico queda recogida en la tabla 27 , donde distinguimos entre los que tienen profilaxis habitual y los que precisan de otros suplementos.

Tabla 27. Pacientes con suplementos habituales en las sucesivas revisiones

\begin{tabular}{|l|c|c|c|c|}
\hline & $\mathbf{1}$ mes & $\mathbf{6}$ meses & $\mathbf{1}$ año & $\mathbf{2}$ años \\
\hline Sí & $297 / 299$ & $267 / 293(91,1 \%)$ & $201 / 287(70 \%)$ & $147 / 286(51,4 \%)$ \\
& $(99,3 \%)$ & & & \\
\hline No & $2 / 299(0,7 \%)$ & $26 / 293(8,9 \%)$ & $86 / 287(30 \%)$ & $139 / 286(48,6 \%)$ \\
\hline
\end{tabular}


En la tabla 28 se muestran los suplementos extra que precisan en cada una de las revisiones.

Tabla 28. Suplementos no habituales en las sucesivas revisiones

\begin{tabular}{|l|c|c|c|c|}
\hline & $\mathbf{1}$ mes & $\mathbf{6}$ meses & $\mathbf{1}$ año & $\mathbf{2}$ años \\
\hline Vitamina D & $2 / 2$ & $13 / 26(50 \%)$ & $31 / 86(36 \%)$ & $51 / 139(36,7 \%)$ \\
\hline Vitamina B12 & - & $7 / 26(27 \%)$ & $27 / 86(31,3 \%)$ & $39 / 139(28 \%)$ \\
\hline Ambas & - & $2 / 26(7,7 \%)$ & $14 / 86(16,3 \%)$ & $29 / 139(20,8 \%)$ \\
\hline Otras & - & $4 / 26(15,3 \%)$ & $14 / 86(16,3 \%)$ & $20 / 139(14,4 \%)$ \\
\hline
\end{tabular}

De la medicación en forma de suplementos el coste de la vitamina $D$ es de 0,9€/cada 15-30 días, y el de la vitamina B12 0.45€/cada 15-30 días.

Entre las otras encontramos vitamina $A$, ácido fólico, vitamina $B 1$ y $E$, calcio, hierro intravenoso y magnesio. El más frecuente el ácido fólico, en seis pacientes, con un coste de 0,09€/día.

\subsubsection{Cuestionario de calidad de vida BAROS}

El cuestionario BAROS a los dos años se realizó a 273/307 pacientes (88,9\%), el resto no acudieron a revisión. De manera global el paciente define su situación como queda reflejado en la tabla 29.

Tabla 29. Definición global de su situación respecto a antes de la cirugía

\begin{tabular}{|l|c|c|}
\hline & Número de pacientes & Porcentaje (\%) \\
\hline Mucho peor & 1 & 0,4 \\
\hline Peor & 1 & 0,4 \\
\hline Igual & 4 & 1,5 \\
\hline Mejor & 46 & 16,8 \\
\hline Mucho mejor & 221 & 81 \\
\hline
\end{tabular}


Por apartados del BAROS vemos las respuestas recogidas en la tabla 30.

Tabla 30. Resultado del cuestionario BAROS por apartados

\begin{tabular}{|l|c|c|c|c|c|}
\hline & $\begin{array}{c}\text { Autoestima } \\
(\%)\end{array}$ & $\begin{array}{c}\text { Actividad } \\
\text { física (\%) }\end{array}$ & $\begin{array}{c}\text { Relaciones } \\
\text { sociales (\%) }\end{array}$ & $\begin{array}{c}\text { Laboral } \\
(\%)\end{array}$ & $\begin{array}{c}\text { Sexual } \\
(\%)\end{array}$ \\
\hline Mucho peor & - & - & - & - & $1(0,4)$ \\
\hline Peor & $1(0,4)$ & - & $1(0,3)$ & $3(1,1)$ & $6(2,2)$ \\
\hline Igual & $14(5,1)$ & $5(1,8)$ & $98(35,9)$ & $100(36,6)$ & $137(50)$ \\
\hline Mejor & $87(31,9)$ & $59(21,6)$ & $87(31,9)$ & $95(34,8)$ & $81(29,8)$ \\
\hline Mucho mejor & $171(62,9)$ & $209(76,6)$ & $87(31,9)$ & $75(27,5)$ & $48(17,6)$ \\
\hline
\end{tabular}

EI PSP para los pacientes con BAROS, dividido en los grupos de puntuación del cuestionario se recoge en la tabla 31.

Tabla 31. Clasificación en grupos de porcentaje de peso perdido (PSP), según el BAROS

\begin{tabular}{|l|c|c|}
\hline PSP & Número de pacientes & Porcentaje (\%) \\
\hline$<25 \%$ & 1 & 0,3 \\
\hline $25-49 \%$ & 29 & 10,6 \\
\hline $50-74 \%$ & 125 & 45,7 \\
\hline$>75 \%$ & 118 & 43,2 \\
\hline
\end{tabular}

En resolución de comorbilidades hay 35 pacientes que no presentaban complicaciones mayores, con lo que la puntuación final del BAROS es sin complicación. De los 238 pacientes que presentaban complicaciones mayores la evolución queda recogida en la tabla 32.

Tabla 32. Evolución de comorbilidades según el cuestionario BAROS $(n=238)$

\begin{tabular}{|l|c|c|}
\hline & Número de pacientes & Porcentaje (\%) \\
\hline Sin cambios & $6 / 238$ & 2,2 \\
\hline Mejorada & $17 / 238$ & 7,1 \\
\hline $\begin{array}{l}\text { Una mayor resuelta otras } \\
\text { mejoradas }\end{array}$ & $61 / 238$ & 25,6 \\
\hline $\begin{array}{l}\text { Todas las mayores resueltas } \\
\text { otras mejoradas }\end{array}$ & $154 / 238$ & 64,7 \\
\hline
\end{tabular}


La evaluación final se recoge en la tabla siguiente.

Tabla 33. Evaluación final del cuestionario BAROS, si presentaba o no comorbilidades previas

\begin{tabular}{|l|c|c|}
\hline & BAROS (\%) & BAROS sin comorbilidades (\%) \\
\hline Regular & $1 / 238(0,4)$ & - \\
\hline Bueno & $44 / 238(18,5)$ & $4 / 35(11,4)$ \\
\hline Muy bueno & $94 / 238(39,5)$ & $18 / 35(51,4)$ \\
\hline Excelente & $99 / 238(41,6)$ & $13 / 35(37,1)$ \\
\hline Puntuación media (DE) & $6,59(1,44)$ & $4,25(0,96)$ \\
\hline
\end{tabular}

Del total de pacientes a los que se realizó el BAROS tenemos que 224/273 $(82 \%)$ de los pacientes tuvieron un resultado muy bueno o excelente.

\subsubsection{Pruebas realizadas}

En la primera revisión (al mes) las pruebas realizadas son de manera selectiva, así se realizaron 2 gastroscopias, una que fue normal y otra que diagnosticó y trató una estenosis de la anastomosis gastro-yeyunal. También se realizó una ecografía abdominal y un TC, que fueron normales.

En la segunda revisión (a los seis meses) fueron necesarias 7 gastroscopias, 5 normales y dos estenosis que se dilataron. Se realizaron 285 estudios gastroduodenales (EGD), de los que en 284 no se objetivó patología subsidiaria de tratamiento, y se detectó una comunicación gastro-gástrica. Se realizaron 24 ecografías: 16 normales, 4 colelitiasis y 4 microlitiasis.

En la tercera revisión (al año) se realizaron 2 gastroscopias diagnósticas, normales, y 2 terapéuticas. EI EGD no presentó hallazgos relevantes en 268 pacientes, detectando 2 estenosis. La ecografía fue normal en 118 pacientes, 25 presentaban colelitiasis, 6 microlitiasis.

En la última revisión (a los dos años) realizamos 4 gastroscopias, 3 normales y una comunicación gastro-gástrica. El EGD se realizó a 254 pacientes, destacando una comunicación gastro-gástrica. Las ecografías fueron normales en 112 pacientes, con 23 colelitiasis y 5 microlitiasis. 


\subsection{Coste de la intervención}

\subsubsection{Contabilidad analítica mediante GRD}

El coste por GRD para el año 2013 viene recogido en la tabla siguiente.

Tabla 34. Costes en función del GRD al alta

\begin{tabular}{|l|c|c|c|c|c|c|}
\hline GRD & Número & $\%$ Global & \% Ajustado & Peso* & $\begin{array}{l}\text { Coste por } \\
\text { unidad de } \\
\text { peso (€) }\end{array}$ & Coste (€) \\
\hline 288 & 269 & 87,6 & 90,9 & 1,6197 & $2.859,54$ & $4.631,6$ \\
\hline 565 & 25 & 8,1 & 8,4 & 4,7414 & $2.859,54$ & 13.558 \\
\hline 877 & 2 & 0,7 & 0,7 & 43,4724 & $2.859,54$ & $124.311,3$ \\
\hline Perdidos & 11 & 3,6 & & & & \\
\hline
\end{tabular}

GRD: Grupos relacionados por el diagnóstico. * Versión 27.0 para el año 2013.

A estos GRD se sumó el del ingreso previo para colocación de BIG en aquellos pacientes que lo precisaron. Es el GRD 297 para todos los casos, que se colocó en 46 pacientes, con un coste de 1.639,37€. En caso del coste individualizado hay que mencionar que el coste del material principal, o sea el balón, fue de $1.683 €$.

En los pacientes que precisaron reingreso en los treinta días posteriores al alta, que fueron dos pacientes, sus GRD fueron el 75, con un coste de 5.171€, y el 154, con un coste de 10.199€, ambos quirúrgicos. Los dos se sumaron al GRD que tenían previamente.

Tras la suma de costes de los GRD para cada uno de los pacientes, sin contar los 11 que no se codificaron, queda una media de 6.545,9 $(10.087,8) €$, rango 4.631,5-124.311,3€. La suma total del coste de todos los GRD es de 1.928.328€. 


\subsubsection{Cálculo individualizado}

El coste individualizado de cada uno de los pacientes intervenidos, que se adjunta en el archivo Excel, nos deja un coste medio de 10.572,2 $(5.485,7) €$, rango de 7.177,5-97.578,92€. La suma total del coste de todos los pacientes es de $3.199 .732,5 €$.

\section{Coste de personal}

El coste de personal incluye el coste de cirujanos, dos adjuntos y un residente por intervención. El de anestesistas, que es un adjunto y un residente. En caso de precisar BIG se suma el coste de un adjunto de endoscopias, un anestesista y un residente. Los costes se detallan en la tabla siguiente.

Tabla 35. Costes de personal para el cálculo individualizado

\begin{tabular}{|l|c|c|c|}
\hline & MIR (€/minuto) & FEA ( $€$ /minuto) & Total. Media (DE) \\
\hline Cirujano & 0,55 & 1,62 & $1.090,3(210,5) €$ \\
\hline Anestesista & 0,55 & 1,28 & $526,4(101,6) €$ \\
\hline Endoscopista & 0,55 & 0,76 & $70,2(26,5) €$ \\
\hline
\end{tabular}

El coste del pase de visita que añadimos en cada día de hospitalización distinto de Reanimación, para cada paciente, es de 40,5€ por paciente y día para el cirujano adjunto, y de 13,75€ para el MIR, considerando que la visita es de un adjunto y un residente, con una media de 12 pacientes valorados en un día.

\section{Coste de farmacia}

El coste de farmacia se calcula en base al gasto que por protocolo consumen los pacientes en el día previo, el de la cirugía y los 5 días posteriores a la intervención (tabla 36), a todo lo que se salga de estos días se le sumará el coste medio diario de farmacia del GFH Cirugía General con Hospitalización. 
Tabla 36. Coste en euros de los fármacos empleados durante el ingreso estándar

\begin{tabular}{|c|c|c|c|}
\hline & Precio (€) & Cantidad & Coste $(€)$ \\
\hline \multicolumn{4}{|l|}{ Día 1: Ingreso } \\
\hline Clexane 40mg subcutáneo & 0,35 & 1 & 0,35 \\
\hline Omeprazol 20mg comprimido oral & 0,38 & 1 & 0,38 \\
\hline Lorazepam $1 \mathrm{mg}$ comprimido sublingual & 0,67 & 1 & 0,67 \\
\hline \multicolumn{4}{|l|}{ Día 2: Intervención Quirúrgica } \\
\hline Suero glucosado $500 \mathrm{cc}$ & 0,7 & 3 & 2,1 \\
\hline Suero Salino 500cc & 0,59 & 2 & 1,18 \\
\hline Paracetamol 1g intravenoso & 0,79 & 3 & 2,37 \\
\hline Omeprazol 40mg intravenoso & 0,39 & 1 & 0,39 \\
\hline Lorazepam $1 \mathrm{mg}$ comprimido sublingual & 0,67 & 1 & 0,67 \\
\hline \multicolumn{4}{|l|}{ Día 3: $1^{\mathrm{er}}$ día postoperatorio } \\
\hline Suero glucosado $500 \mathrm{cc}$ & 0,7 & 3 & 2,1 \\
\hline Suero Salino $500 \mathrm{cc}$ & 0,59 & 2 & 1,18 \\
\hline Desketoprofeno 50mg intravenoso & 0,33 & 3 & 0,99 \\
\hline Clexane $40 \mathrm{mg}$ subcutáneo & 0,35 & 1 & 0,35 \\
\hline Paracetamol 1g intravenoso & 0,79 & 3 & 2,37 \\
\hline Omeprazol 40mg intravenoso & 0,39 & 1 & 0,39 \\
\hline Metoclopramida $10 \mathrm{mg}$ intravenoso & 0,15 & 3 & 0,45 \\
\hline Lorazepam $1 \mathrm{mg}$ comprimido oral & 0,67 & 1 & 0,67 \\
\hline \multicolumn{4}{|l|}{ Día 4: 2 día postoperatorio } \\
\hline Suero glucosado $500 \mathrm{cc}$ & 0,7 & 3 & 2,1 \\
\hline Suero Salino 500cc & 0,59 & 2 & 1,18 \\
\hline Paracetamol 1g intravenoso & 0,79 & 3 & 2,37 \\
\hline Metamizol $2 \mathrm{~g}$ intravenoso & 0,24 & 3 & 0,72 \\
\hline Omeprazol 20mg comprimido oral & 0,38 & 1 & 0,38 \\
\hline Clexane 40mg subcutáneo & 0,35 & 1 & 0,35 \\
\hline \multicolumn{4}{|l|}{ Día 5: $3^{\text {er }}$ día postoperatorio } \\
\hline Paracetamol 500mg vía oral & 0,15 & 6 & 0,9 \\
\hline Omeprazol 20mg comprimido oral & 0,38 & 1 & 0,38 \\
\hline Clexane $40 \mathrm{mg}$ subcutáneo & 0,35 & 1 & 0,35 \\
\hline \multicolumn{4}{|l|}{ Día 6: 4ํ día postoperatorio } \\
\hline Paracetamol 500mg vía oral & 0,15 & 6 & 0,9 \\
\hline Omeprazol 20mg comprimido oral & 0,38 & 1 & 0,38 \\
\hline Clexane $40 \mathrm{mg}$ subcutáneo & 0,35 & 1 & 0,35 \\
\hline Día 7: 5 día postoperatorio ALTA & nada & & 1,63 \\
\hline \multicolumn{3}{|c|}{ TOTAL DE CONSUMO DE FÁRMACOS DURANTE EL INGRESO } & $28,6 €$ \\
\hline
\end{tabular}

\section{CONSUMO MEDIO FÁRMACOS DEL SERVICIO DE CIRUGÍA $15,64 € /$ día GENERAL / ESTANCIA EN EL AÑO 2013}

El coste medio en fármacos de los pacientes intervenidos fue de 104 (98)€. 


\section{Coste de suministros específicos}

El material empleado en la cirugía se detalla en la tabla siguiente.

Tabla 37. Material quirúrgico empleado en el BPG laparoscópico

\begin{tabular}{|c|c|c|}
\hline MATERIAL & $\begin{array}{c}\text { PRECIO } \\
(€)^{\star}\end{array}$ & $\begin{array}{c}\text { UNIDADES } \\
\text { EMPLEADAS }\end{array}$ \\
\hline Bisturí Harmónico & 577,5 & 1 \\
\hline Trócares Xcel Optiview Ethicon 12mm & 100,75 & 1 \\
\hline Trócar Xcel sin introductor $12 \mathrm{~mm}$ & 51,4 & 4 \\
\hline Aspirador: mango & 172,08 & 1 \\
\hline terminal & 87,38 & 1 \\
\hline Endostitch: porta & 222,05 & 1 \\
\hline sutura seda $2 / 0$ & 26,19 & 5 \\
\hline Pinza Endopath & 121,1 & 1 \\
\hline Tijera & 83,51 & 1 \\
\hline Pinza Anvil Grasper & 201,98 & 1 \\
\hline Echelon pistola & 401,5 & 1 \\
\hline Sutura mecánica 60mm carga 2,5mm & 209 & 4 \\
\hline Sutura mecánica 60mm carga 3,5mm & 209 & 4 \\
\hline Sutura mecánica circular CEEA 25 & 278,04 & 1 \\
\hline Sutura Vicryl 1 anzuelo & 4,36 & 1 \\
\hline Aspirador de humo & 30,06 & 1 \\
\hline Aguja de Veress & 10,28 & 1 \\
\hline Sutura Monocryl $3 / 0$ & 3,58 & 1 \\
\hline Sutura Prolene 0 & 2,77 & 1 \\
\hline Sonda nasogástrica $n^{\circ} 16$ & 1,13 & 1 \\
\hline Sonda calibradora $34 \mathrm{fr}$ & 14,14 & 1 \\
\hline Campo estéril & 29,4 & 1 \\
\hline Endobag & 127,71 & 0 ó 1 \\
\hline Endoclips $10 \mathrm{~mm}$ & 129,86 & 0 ó 1 \\
\hline Grapadora piel & 7,3 & 1 \\
\hline Filtro de insuflación antibacteriano & 10,6 & 1 \\
\hline Sonda drenaje Jackson-Pratt & 33,88 & 1 \\
\hline Colector de drenaje $400 \mathrm{cc}$ & 20,75 & 1 \\
\hline BIG & 1.683 & 0 ó 1 \\
\hline
\end{tabular}

${ }^{*}$ Precio medio del año 2013. CEEA: Anastomosis mecánica circular

El de la cirugía abierta es similar, descontando el material propio de la laparoscopia. 
El coste en material para cirugía abierta fue de 3.077,57€ y laparoscópica de $4.442,42 €$. En los casos que hubo complicación con reintervención la media de la cirugía fue de $3.259,28 €$. Se empleó material de cirugía programada y otros que se detallan en la tabla siguiente.

Tabla 38. Material quirúrgico empleado en reintervenciones

\begin{tabular}{|l|c|c|}
\hline \multicolumn{1}{|c|}{ Material } & $\begin{array}{c}\text { PRECIO } \\
(\boldsymbol{\epsilon})^{\star}\end{array}$ & $\begin{array}{c}\text { UNIDADES } \\
\text { EMPLEADAS }\end{array}$ \\
\hline Sutura Polipropileno 0 & 2,78 & 3 \\
\hline Sutura Ácido.Poliglicólico 0 & 3,47 & 3 \\
\hline Equipo catéter venoso central: dos vías cánula 7ch & 71,29 & 1 \\
\hline Malla de Polipropileno y Poliglactina 15x15cm & 151,18 & 1 \\
\hline $\begin{array}{l}\text { Sonda gastrostomía endoscópica percutánea silicona } \\
\text { dos vías con balón 24ch }\end{array}$ & 70,58 & 1 \\
\hline
\end{tabular}

El coste medio de material quirúrgico fue de $3.897,9(753,6) €$.

\section{Costes de Anatomía Patológica}

El coste del análisis anatomopatológico de la vesícula biliar fue de $30,8 €$.

\section{Coste de laboratorio}

El coste de laboratorio para una analítica básica fue de 34,95€, y el de analítica completa de 54,95€. Se considera que en un ingreso se realiza una analítica básica cada 4 días y una completa semanal, donde se incluyen parámetros nutricionales, vitaminas, y pruebas de función hepática, de los que más llama la atención la pre-albúmina, con un coste de 9,6€. El coste queda incluido en el coste por estancia. 


\section{Coste de Unidades de Enfermería}

El coste de enfermería se incluye en el coste de la planta, que para la planta del Servicio de Cirugía General donde ingresan, fue de $125 €$ por día. El coste medio fue de $1.402,6(3.012,1) €$.

\section{Coste de quirófano}

El coste de quirófano, en el que se incluye tanto medicación, material utilizado por Anestesia, y personal no facultativo del Área Quirúrgica, fue para el quirófano utilizado para la cirugía bariátrica, de 6,54€/minuto. El coste medio fue de $3.574,6(691,5) €$.

\section{Coste de Reanimación}

Es un coste único, por paciente, independiente del número de días de ingreso que precise. El coste fue de $362,6 €$ para todos los pacientes y contabilizado por cada vez que van a quirófano.

\section{Coste de Radiodiagnóstico}

Las pruebas realizadas durante el ingreso se recogen en la tabla 39. Consideramos que un paciente con ingreso superior a las dos semanas realizará un TC abdominal por cada dos semanas más de ingreso. Una radiografía de tórax cada 4 días de ingreso. El estudio gastroduodenal se realiza el día siguiente a la cirugía. El coste queda incluido en el coste por estancia.

Tabla 39. Costes de pruebas de imagen durante el ingreso

\begin{tabular}{|l|c|}
\hline \multicolumn{1}{|c|}{ Tipo } & Precio $(\boldsymbol{\epsilon})$ \\
\hline Radiografía de Tórax & 12,23 \\
\hline TC Abdómino-pélvico con contraste & 158,66 \\
\hline Estudio Gastroduodenal & 62,75 \\
\hline
\end{tabular}




\section{Coste de alimentación}

La media de gasto en dieta se recoge en coste de hostelería por estancia, que para la planta de Cirugía General fue de 30,8€/día. Dado que la ingesta básicamente son caldos, y que no todos los días hay ingesta se desestima el uso de este coste.

\section{Otros costes}

Si tenemos en cuenta los costes preoperatorios tanto en consultas como en pruebas solicitadas, así como los costes postoperatorios, ajenos al ingreso, pero que están directamente relacionados con la cirugía, debemos incluir los siguientes:

Tabla 40. Costes de estudios y consultas perioperatorias

\begin{tabular}{|l|c|}
\hline Costes de estudios & PRECIO (€) \\
\hline & \\
\hline Gastroscopia & 205,48 \\
\hline Ecografía Abdómino-pélvica & 40,26 \\
\hline Estudio Gastroduodenal & 62,75 \\
\hline Polisomnografía del sueño & 426 \\
\hline Electrocardiograma & 14,86 \\
\hline Ecocardiograma & 87,3 \\
\hline Espirometría & 39,64 \\
\hline Analítica básica & 34,95 \\
\hline & \\
\hline Costes de consultas & 130,51 \\
\hline & 136,76 \\
\hline Primera consulta Cirugía General & 128,52 \\
\hline Primera consulta Endocrino & 349,49 \\
\hline Primera consulta Salud Mental & 67,05 \\
\hline Primera consulta Neumología & 78,31 \\
\hline Primera consulta Anestesia & 82,06 \\
\hline Consulta sucesiva Cirugía General & \\
\hline Consulta sucesiva Endocrino & \\
\hline
\end{tabular}


El coste medio de estudios y consultas realizados antes de la cirugía fue de $1.708,2(83,9) €$. El coste medio de estudios y consultas realizados tras la cirugía fue de 833,6 $(30,4) €$.

En este apartado debemos hacer mención a la inversión original que fue necesaria para comenzar con el programa de cirugía bariátrica en el HUCA, por las necesidades especiales de estos pacientes, por un total de $125.456 €$, como viene detallado en la siguiente tabla.

Tabla 41. Inversión inicial para iniciar el programa de cirugía bariátrica

\begin{tabular}{|c|c|c|c|c|}
\hline Año & MATERIAL & UNIDADES & PRECIO (€) & TOTAL $(€)$ \\
\hline 2002 & Mesa bariátrica & 1 & $18.454,18$ & $18.454,18$ \\
\hline 2002 & Báscula para obesos & 1 & 458,59 & 458,59 \\
\hline 2003 & Accesorios para mesa & 1 & $9.134,10$ & $9.134,10$ \\
\hline 2003 & Cama para obesidad & 2 & $2.767,50$ & 5.535 \\
\hline 2003 & Báscula con plataforma & 1 & 2.401 & 2.401 \\
\hline 2003 & $\begin{array}{l}\text { Separador quirúrgico } \\
\text { especial }\end{array}$ & 1 & $11.905,71$ & $11.905,71$ \\
\hline 2003 & Laringoscopio & 1 & 179,76 & \multirow[t]{2}{*}{664,15} \\
\hline 2003 & Pala de luz fría & 1 & 484,39 & \\
\hline 2003 & Monitor de anestesia & 1 & 10.730 & 10.730 \\
\hline 2003 & $\begin{array}{l}\text { Set de anestesia y } \\
\text { reanimación }\end{array}$ & 1 & 10.250 & 10.250 \\
\hline 2003 & Silla en tubo de acero & 4 & 105 & 420 \\
\hline 2003 & Colchón antiescaras & 2 & 593,85 & $1.187,70$ \\
\hline 2004 & Bipap visión & 1 & 11.750 & 11.750 \\
\hline 2004 & $\begin{array}{l}\text { Soporte móvil para v. no } \\
\text { invasiva }\end{array}$ & 1 & 5.250 & 5.250 \\
\hline 2004 & Respirador & 1 & 21.000 & 21.000 \\
\hline 2004 & Grúa liko viking 300 & 1 & 6.205 & \multirow[t]{3}{*}{8.231} \\
\hline 2004 & Kit bascula para grúa liko & 1 & 1.810 & \\
\hline 2004 & Arnés & 1 & 216 & \\
\hline 2005 & Silla de ruedas & 2 & 782,7 & $1.565,40$ \\
\hline 2005 & Grúa & 1 & 6.520 & 6.520 \\
\hline
\end{tabular}




\section{Costes totales}

El coste de cada uno de los apartados mencionados, para el total de la serie deja los siguientes consumos:

\section{- Área quirúrgica}

El coste medio (DE) del grupo de cirujanos fue de 1.090,3 (210,5)€, de anestesistas de 526,4 (101,6)€. En los que precisaron BIG el coste medio de endoscopista fue de 40,7 (15,3)€.

El material quirúrgico para la cirugía abierta fue de 3.077,57€ y laparoscópica $4.442,42 €$ y en caso de precisar colecistectomía en la laparoscópica sumaremos $257,27 €$. En aquellos que precisaron conversión se asume el coste de la cirugía laparoscópica. En los que tuvieron un BIG previo se sumó el coste del mismo (1.683€ por balón). El coste medio de material fue de 3.897,9 $(753,6) €$.

El coste medio de utilización de quirófano fue de 1.870,2 $(392,8) €$. Si incluimos material y personal facultativo y estancia en Reanimación, nos queda un coste de intervención medio de $6.211,8(3.551,3) €$. Aquellos que tuvieron un BIG previo a la intervención, el coste medio de quirófano para su colocación incluido el personal fue de 489,1 (184,6)€, que junto al material utilizado ya está incluido en el coste medio de intervención.

\section{- Área de hospitalización}

El coste medio de hospitalización, que incluye el coste de Unidad de Enfermería, y los correspondientes al consumo durante el periodo de hospitalización de Farmacia, Laboratorio y Radiodiagnóstico, fue de 2.205,6 $(1.296,1) €$.

\section{- Ingreso}

En los que precisaron un BIG previo el coste de ese ingreso fue de 2.944,8 $(184,6) €$, que incluimos en el coste total del ingreso. 
El coste del ingreso, incluidas las complicaciones que precisaron reintervención con o sin reingreso, fue de $10.572,2(5485,7) €$.

\section{- Todo el proceso}

Incluyendo los estudios y consultas preoperatorias, que tuvieron una media de $1.708,2(83,9) €$, nos queda un coste al momento del alta de 12.280,5 (5.497)€. Si queremos saber el coste de todo el periodo a estudio de 2 años incluimos las consultas y pruebas realizadas en el seguimiento postoperatorio, y nos queda una media de 13.095 (5.502)€.

Si comparamos ambos modelos de estimación de costes (tabla 42) para los distintos grupos de peso y abordaje quirúrgico, encontramos diferencias en todos ellos, la más llamativa la de la cirugía laparoscópica, que es más del doble.

Tabla 42. Comparación de costes por abordaje y peso, recogido en la media en euros

\begin{tabular}{|l|c|c|c|}
\hline & Coste por GRD & Coste por ingreso & p \\
\hline Cirugía abierta & $7.234,1$ & $10.354,3$ & $<0.001$ \\
\hline Cirugía laparoscópica & $5.293,9$ & $10.968,1$ & $<0.001$ \\
\hline IMC $<45$ & $5.286,8$ & $10.427,1$ & $<0.001$ \\
\hline IMC 46-55 & $7.106,4$ & $10.509,8$ & $<0.001$ \\
\hline IMC $>55$ & $6.638,2$ & $10.932,5$ & $<0.001$ \\
\hline
\end{tabular}

GRD: Grupos relacionados por el diagnóstico. IMC: Índice de masa corporal.

El coste por ingreso es más homogéneo en sus resultados, ya que mientras en la cirugía laparoscópica se incrementa el coste del material y tiempo quirúrgico, se ve compensado por un ingreso menos prolongado que la cirugía abierta.

Esta capacidad de adaptación o de análisis detallado que no tiene el GRD le lleva a tener un menor coste medio en la cirugía laparoscópica frente a la abierta $(p=0,037)$, por tener más GRD 288 en este grupo, que tienen menos coste. Lo mismo sucede con el IMC menor de 45, y su menor coste $(p=0,008)$, por el mismo fenómeno ya que en este grupo la mayoría fueron GRD 288 y no hubo ningún GRD 877. 


\subsection{Análisis estadístico}

Una vez definidos los parámetros clínicos y económicos procedemos a realizar el análisis para conocer el comportamiento de la serie, recogido en la tabla 43. Para ello comenzamos por la parte clínica detallando las variables de efectividad que definen esta intervención.

De manera global el BAROS reúne las tres variables de efectividad que nos interesan (pérdida de peso, resolución de comorbilidades y calidad de vida), pero debemos diferenciar entre aquellos con comorbilidades y los que no las tienen, ya que puntúan de manera distinta. Estudiamos las variables demográficas, IMC en primera consulta, comorbilidades mayores (HTA, DM, DL SAHS, Artrosis y Depresión), BIG previo, tipo de abordaje, estancia, y si hubo complicaciones que demoraron el alta.

No consideramos en el análisis 1 paciente con resultado BAROS regular, de los 238 pacientes con BAROS del grupo que presentaban complicaciones, por la distorsión que provocaba en el análisis.

En los 237 pacientes a los que se realizó el BAROS a los dos años, con comorbilidades previas, fue la edad con una $p=0,004$ la que mostró en pacientes más jóvenes un resultado excelente. En los que tuvieron complicaciones que demoraron el alta el resultado del BAROS fue peor $(p=0,017)$. En cuanto a las comorbilidades previas fueron la depresión $(p=0,01)$ y la artrosis $(p=0,06)$ las que tuvieron más influencia, presentando resultados peores en aquellos que las padecían.

En los 35 pacientes sin comorbilidades previas realizamos el mismo análisis. En este grupo vemos que los pacientes con IMC más bajo presentaban más resultados excelentes $(p=0,072)$. La edad, sin tener tampoco significación, tuvo una diferencia de años entre grupos que fue prácticamente igual al grupo anterior, con preferencia por los jóvenes con mejores resultados $(p=0,305)$. En cuanto al sexo sólo hubo un hombre en este grupo. Con complicación sólo fueron seis pacientes y con BIG cinco, pero sólo hubo un resultado excelente entre los dos grupos con unas diferencias $p=0,145$ y $p=0,177$ respectivamente, lo que quizá orienta a tener alguna significación si la muestra fuese mayor. 
Pasamos al PSP como variable de efectividad, y categorizamos según los mismos grupos que hace el BAROS, por no existir consenso a la hora de definir el éxito, con un resultado excelente en aquellos en los que el PSP a los dos años es mayor del $75 \%$, y para las mismas variables estudiadas previamente. Así se obtuvieron diferencias significativas entre las distintas categorías establecidas en el PSP para las variables edad $(p<0,001)$ e IMC en primera consulta $(p=0,01)$, en favor de los jóvenes e IMC bajo. Los pacientes con BIG no consiguieron tantos resultados excelentes como los que no lo necesitaron $(p=0,001)$. Los pacientes con HTA $(p=0,08)$, DM $(p=0,001)$, artrosis $(p=0,018)$ y depresión $(p=0,01)$ obtuvieron peores resultados en PSP respecto a los que no las padecían.

En la resolución de comorbilidades, el resultado excelente es el que tiene todas las mayores resueltas y otras mejoradas. Son los pacientes más jóvenes los que consiguen este resultado $(p=0,024)$. Por comorbilidades, los pacientes con DM $(p=0,038)$ y SAHS $(p=0,007)$, presentan un mejor resultado que los que no los padecen, mientras que los pacientes que no tienen artrosis serán los de mejor resultado $(p<0,001)$.

Si analizamos calidad de vida, con resultado mayor de 1,5 como excelente, fueron sólo los pacientes con depresión los que se vieron influidos. Los que no tenían depresión fueron los que mejor resultado tuvieron $(p=0,011)$.

Para el coste hicimos uso del coste final del ingreso con las dos modalidades, uno por GRD, y otro el individualizado. Así diferenciamos ambos en dos grupos, en función de la mediana, que para el GRD estuvo en 4.631€ y para el coste individual en 9.936€.

El coste por GRD, para las mismas variables de análisis realizadas en el apartado anterior, resulta más caro en pacientes con IMC más elevado $(p<0,001)$, en los que presentaron complicación o necesitaron un $\mathrm{BIG}(p<0,001)$ el precio fue mayor, y más barato en los sometidos a laparoscopia $(p<0,001)$. Para el resto de variables no hubo relación estadísticamente significativa.

El coste por paciente muestra diferencias en el IMC. Más barato en los IMC más bajos $(p=0,041)$, y en los de menor estancia $(p=0,045)$. Entre los más 
caros estuvieron aquellos con complicaciones $(p<0,001)$, los que tuvieron un BIG $(p<0,001)$, los de abordaje laparoscópico $(p<0,001)$ y en cuanto a comorbilidades, los que tenían SAHS $(p=0,021)$ fueron más caros, hay que señalar que la media (DE) del IMC para los que tenían SAHS fue de 50,6 $(7,1)$ $\mathrm{kg} / \mathrm{m}^{2}$ frente a los $48,8(6,2) \mathrm{kg} / \mathrm{m}^{2}$ de los que no $(p=0,02)$. 


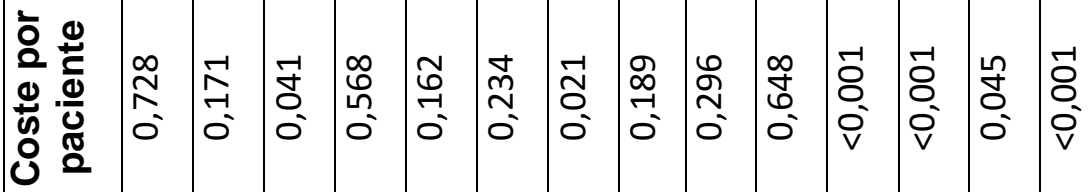

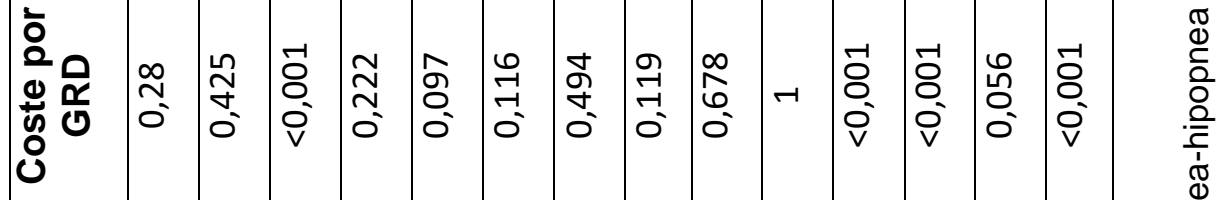

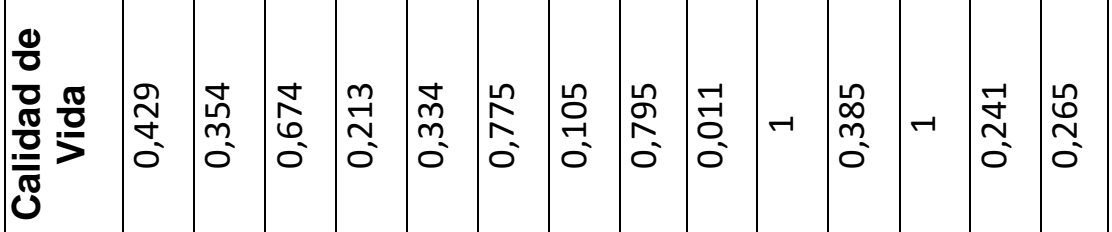

$\stackrel{2}{8}$

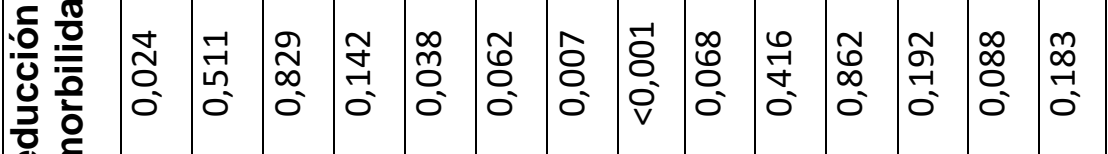

寗高

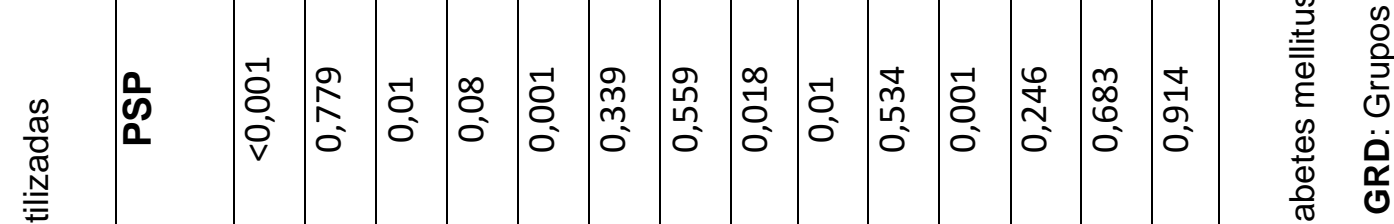

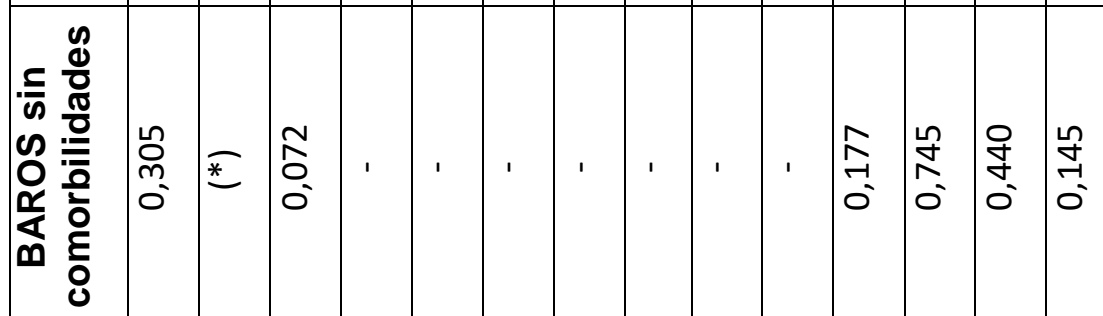

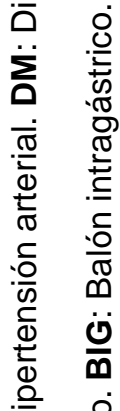

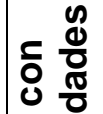

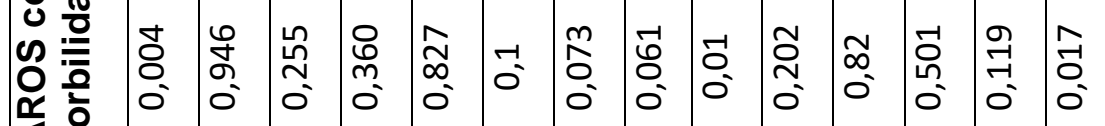

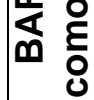


Para el estudio coste-efectividad de la cirugía abierta frente a la laparoscópica usamos los valores de efectividad descritos, para cada uno de los métodos de cálculo de coste del ingreso, quedando recogidas las medias en la siguiente tabla.

Tabla 44. Medias de coste-efectividad de la cirugía abierta vs laparoscópica

\begin{tabular}{|l|c|c|c|}
\hline & Cirugía abierta & Cirugía laparoscópica & p \\
\hline BAROS por GRD & 275,1 & 207,6 & 0,108 \\
BAROS por coste individual & 393,2 & 430,2 & 0,099 \\
\hline PSP por GRD & 107,8 & 78,7 & 0,053 \\
PSP por coste individual & 164,5 & 166 & 0,882 \\
\hline CV por GRD & $4.293,1$ & $2.974,6$ & 0,096 \\
CV por coste individual & $6.335,2$ & $6.228,7$ & 0,912 \\
\hline RC por GRD & $2.784,5$ & $2.077,9$ & 0,036 \\
RC por coste individual & $4.318,3$ & $4.367,9$ & 0,843 \\
\hline \multicolumn{2}{|c|}{ GRD: Grupos relacionado por el diagnóstico. PSP: Porcentaje de sobrepeso perdido. CV. }
\end{tabular}

GRD: Grupos relacionado por el diagnóstico. PSP: Porcentaje de sobrepeso perdido. CV:

Calidad de vida. RC: Resolución de comorbilidades. BAROS: Cuestionario de calidad de vida.

El resultado BAROS como agrupación de todas las variables que persigue esta cirugía nos parece el método más correcto para conocer si es la cirugía abierta o la laparoscópica la que se muestra más coste-efectiva. Para el coste medio por GRD la relación CE de cirugía abierta frente a laparoscópica fue 275,1 vs 207,6 $(p=0,1)$, con la premisa de menos es más, o sea el cociente nos muestra el mejor resultado de manera inversa, este fue favorable a la laparoscópica.

Para el coste medio por paciente la relación CE de cirugía abierta frente a laparoscópica fue de 393,2 vs $430,2(p=0,09)$, siendo en este caso favorable a la cirugía abierta, por ser esta más barata, y no llegar el BAROS a compensarlo, ya que a pesar de ser mejor en la laparoscopia, la diferencia de este por el tipo de abordaje es pequeña.

El resto de resultados reflejan un coste más bajo de la laparoscopia por GRD, y alcanza significación en la resolución de comorbilidades. 


\subsubsection{Pacientes con DM}

Ya que en apariencia los grandes beneficiados de la cirugía bariátrica, en lo clínico y en lo económico son los diabéticos, hacemos hincapié a continuación en este punto. En la siguiente tabla quedan recogidas las diferencias clínicas en los pacientes diabéticos.

Tabla 45. Características de los pacientes diabéticos

\begin{tabular}{|l|c|c|c|}
\hline & DM (n=85) & No DM (n=222) & p \\
\hline Edad media (DE) en años & $49,4(7,8)$ & $41,2(9,8)$ & $<0,001$ \\
\hline Mujeres (\%) & $65(76,5)$ & $174(78,4)$ & 0,719 \\
\hline Comorbilidades (\%) & $84(98,8)$ & $199(89,6)$ & 0,007 \\
\hline HTA (\%) & $70(82,4)$ & $94(42,3)$ & $<0,001$ \\
\hline DL (\%) & $43(50,5)$ & $32(14,4)$ & $<0,001$ \\
\hline SAHS (\%) & $61(71,8)$ & $117(52,7)$ & 0,002 \\
\hline Artrosis (\%) & $43(50,5)$ & $64(29)$ & $<0,001$ \\
\hline Colelitiasis (\%) & $23(27)$ & $28(12,6)$ & 0,002 \\
\hline Depresión (\%) & $43(50,5)$ & $81(36,5)$ & 0,039 \\
\hline ERGE (\%) & $23(27)$ & $54(24,3)$ & 0,621 \\
\hline IMC 1 Consulta (DE) en kg/m ${ }^{2}$ & $50,1(7,1)$ & $49,8(6,7)$ & 0,74 \\
\hline BIG (\%) & $17(20)$ & $29(13,1)$ & 0,128 \\
\hline Estancia media (DE) en días & $15,3(44,6)$ & $9,6(6)$ & 0,24 \\
\hline Cirugía laparoscópica (\%) & $25(29,4)$ & $84(37,8)$ & 0,167 \\
\hline Conversiones (\%) & $4(4,6)$ & $5(2,3)$ & 0,241 \\
\hline Complicaciones demoran el alta (\%) & $13(15,3)$ & $28(12,6)$ & 0,537 \\
\hline Coste medio por GRD en $€$ & $8.658,6$ & $5.722,6$ & 0,253 \\
\hline Coste medio por paciente en $€$ & 11.455 & $10.234,2$ & 0,081 \\
\hline
\end{tabular}

IMC: Índice de masa corporal. HTA: Hipertensión arterial. DM: Diabetes mellitus. DL: Dislipemia. SAHS: Síndrome de apnea hipopnea del sueño. ERGE: Enfermedad por reflujo gastroesofágico. IMC: Índice de masa corporal. BIG: Balón intragástrico. GRD: Grupos relacionados por el diagnóstico. DE: Desviación estándar. 



\section{DISCUSIÓN}





\section{DISCUSIÓN}

\subsection{La cohorte}

\subsubsection{Parámetros demográficos y antropométricos}

En el año 2006 la OMS en la Carta Europea contra la Obesidad fija como objetivo revertir la tendencia en el año 2015 (165), y aquí estamos, peor que hace 10 años. La obesidad es un problema de salud pública de primer orden y por el cual debemos hacer un esfuerzo intenso en su prevención, así como conocer el coste de ese empleo de recursos.

En este sentido hay que entender la eficacia como una obligación moral de los médicos, pero también debe serlo de los gestores, y no impedir medidas encaminadas a retomar la salud de nuestra sociedad, por razones puramente económicas, al amparo de la eficiencia. En cualquier caso hay que procurar perseguir ambos objetivos.

Los resultados de nuestra serie nos muestran una población con una edad media de 43,5 años, con $78 \%$ de mujeres y un IMC medio de $49,9 \mathrm{~kg} / \mathrm{m}^{2}$; similares a series previas, como el meta-análisis de Buchwald (2), el artículo más citado de la historia de la cirugía bariátrica (166), con 38,9 años de edad media, un $72,6 \%$ de mujeres y un IMC medio de $46,8 \mathrm{~kg} / \mathrm{m}^{2}$.

\subsubsection{Comorbilidades relacionadas con la obesidad}

\section{HTA}

Partimos de un 53,4\% de HTA en nuestra serie, con una evolución en dos años que consigue una resolución en el $75 \%$ de los pacientes, lo que implica que no toman medicación, y una mejoría en el $21 \%$, quedando solo cinco pacientes (3\%) al año y a los dos años que están igual. Resultados similares a los de Buchwald (2), con un $87 \%$ de resolución o mejoría, y mejores que el $79 \%$ de la serie de Hutter, al año de la cirugía (167). 
Los fármacos más utilizados en el tratamiento de la HTA se recogen en la tabla 46 , con un coste anual superior a los $400 €$ en la mayoría de ellos (168). Es complejo hacer un seguimiento exacto de la medicación de nuestros pacientes, por la gran variedad de fármacos del mercado y la rotación que existe entre ellos. Para nuestra valoración económica haremos uso de los resultados de Sicras et al que lo sitúan en $894 €$ anuales (156).

Tabla 46. Coste de fármacos antihipertensivos en España. Tomado de García-Ruíz (168)

\begin{tabular}{|c|c|c|c|c|}
\hline Fármacos & Dosis PA (mg) & N. de comprimidos & PVP + IVA (E) & Coste anual $(€)$ \\
\hline \multirow[t]{2}{*}{ Amlodipino/Otmesartán } & $5 / 20$ & 28 & 27,91 & 408,36 \\
\hline & $10 / 40$ & 28 & 40,64 & 529,77 \\
\hline \multirow[t]{2}{*}{ Amlodipino/Valsartán } & $5 / 160$ & 28 & 37,40 & 487,54 \\
\hline & $10 / 160$ & 28 & 40,60 & 529,25 \\
\hline Manidipino/Delapril & $10 / 30$ & 28 & 21,07 & 274,66 \\
\hline
\end{tabular}

\section{DM}

El 27,7\% en nuestra serie tenían DM al ingreso, con una evolución que consiguió resolución al mes en el $60 \%$ y del $89 \%$ de los pacientes en dos años, lo que implica que no toman medicación, y una mejoría en el 32 y $11 \%$ respectivamente, y todos los pacientes al año y a los dos años estaban mejor que antes de la intervención. Unos resultados mejores que los de Buchwald (2) y Hutter (167) con un 93\% y 83\% respectivamente de resolución o mejoría.

En el caso de los pacientes con DM hay publicaciones que encuentran en el tratamiento con BPG una relación coste-efectiva (131), para un coste por intervención de 8.344€, muy por debajo del coste generado por nuestros pacientes si hacemos uso de los GRD, y un coste aproximado si hacemos uso del coste por paciente. Con un gasto en fármacos estimado de $1.661 €$ anuales con el tratamiento convencional en España (169) en un estudio de varios países europeos, y de $1.770 €$ anuales en un estudio nacional (157), que representa en torno al $15 \%$ del coste total. 


\section{DL}

Partimos de 73 pacientes, el 23,7\% en nuestra serie con DL, y conseguimos la resolución del $88,4 \%$ de los pacientes en dos años, lo que implica que no toman medicación, y una mejoría en el 8,7\%, quedando dos pacientes igual que antes de la cirugía a los dos años. Resultados similares al $94 \%$ de resolución o mejoría de Buchwald (2), y mejor que el 66\% de resolución la serie de Hutter, al año de la cirugía (167) o el 70\% de Schauer (164). El cálculo económico lo hacemos con un coste anual de $282 €(158)$.

\section{SAHS}

178/307 pacientes padecían SAHS, de los que el 98\% hacían uso de la CPAP. En las sucesivas revisiones, al mes ya tenemos un 27,3\% de resolución, a los 6 meses $63,2 \%$, al año $80,1 \%$ y a los dos años $89,6 \%$. En cuanto al uso de la CPAP, al mes la usaban el $68 \%$, a los 6 meses el $34 \%$, al año el $18 \%$, y a los dos años el 9\%, una disminución progresiva en torno a la mitad de las previas.

Resultados mejores al estudio de Hutter (167) en el que el 66\% los pacientes al año presentan resolución. Y similares a los de los meta-análisis de Buchwald (2) con un $80 \%$ de resolución, y Lopes (135) con un 5,3\% que mantiene la CPAP.

Traducido en coste, la terapia CPAP se calcula en unos $675 €$ anuales $(1,85 €$ por sesión) (159), coste que ayuda a amortizar la inversión inicial que significa la cirugía, y que en el $90 \%$ de los pacientes que la padecían, se resuelve.

\section{Artrosis}

Aunque partimos de un $34,8 \%$ de artrosis en nuestra serie, tan solo en 83 de los 107 pacientes $(77,5 \%)$ tenemos recogida la medicación, por un lado por la percepción de no ser una patología directamente relacionada con la obesidad, y por otro por lo cambiante de la medicación que toman. La evolución en dos años consigue una resolución en el $32 \%$ de los pacientes, lo que implica que no toman medicación, y una mejoría en el 56\%. El $82 \%$ de este grupo son mujeres. Al igual que la depresión no están contemplados en los resultados de 
las grandes series, entre otras razones por lo difícil de objetivarlo. En nuestra serie viene definido por la ausencia de medicación, tanto profiláctica como puede ser el calcio, como terapéutica como los AINES; y no referir el paciente que lo padece.

En el caso de la artrosis de rodilla y cadera, zonas más afectadas por la obesidad, representa un coste anual en España es de 4.700 millones de euros, de los cuales, el 46 por ciento corresponde a gastos asistenciales, el 22 por ciento a bajas laborales, el 13 por ciento a ingresos hospitalarios, el 7 por ciento a pruebas diagnósticas y el 5 por ciento a fármacos (170). Es difícil evaluar la mejora que supuso esta intervención ya que no disponemos de cuestionarios específicos, en cualquier caso la pérdida de peso supone una mejoría directa sobre esta patología, y sin duda un ahorro, aunque no nos atrevemos a cuantificarlo.

\section{Depresión}

Al igual que la artrosis es difícil su evaluación. Partimos de un $40 \%$ de depresión en nuestra casuística, con una evolución en dos años que consigue una resolución en el $44 \%$ de los pacientes, lo que implica que no toman medicación, y una mejoría en el 42\%, quedando un $12 \%$ que continúan igual. No es una patología que se analice habitualmente a la hora de valorar resolución de comorbilidades, y se sabe que no responde igual de bien que otras más vinculadas a fenómenos metabólicos. Encontramos un estudio en el que al año tan solo encuentran un $9 \%$ que no precise medicación (171), muy por debajo de nuestro 38\%. Para definir su resolución, a falta de haber realizado cuestionarios específicos de la patología, valoramos la ausencia de medicación tanto antidepresiva como ansiolítica, y que no quede reflejada su presencia en la hoja de seguimiento.

De la depresión, según un estudio (160) publicado en el 2010, la diferencia de coste entre pacientes que presentaban remisión, respecto a los que no, era de casi $1.000 €$ anuales, lo cual sigue sumando ahorro. 


\section{Otras}

La $25,1 \%$ de los pacientes presentaba ERGE, y si bien algún paciente sigue con la ingesta de inhibidores de la bomba de protones tras la cirugía, por más de un mes que es lo que indicamos de manera general, no es motivo de revisión en este estudio, considerando que esta cirugía resuelve tanto el reflujo ácido como el biliar.

La colelitiasis, relacionada con la obesidad y con el sexo femenino, y que afecta entre un 10-15\% de la población (172), en nuestra serie está presente en el 16,6\%, presenta porcentajes similares a otras publicaciones (173), si bien nuestra cohorte es de predominio femenino y obeso, la del estudio mencionado con la mitad de la serie varones, tiene una mayor edad media. En cualquier caso la patología queda resuelta en la misma intervención para aquellos con IMC mayor de $55 \mathrm{~kg} / \mathrm{m}^{2}$ o que tienen colelitiasis en la ecografía preoperatoria, lo que previene futura patología biliar, que implica coste sanitario. En total se realizó colecistectomía en un $31,6 \%$ de los pacientes. Si asumimos que la incidencia anual de complicación es de un 0,3\% (174), que un 50\% desarrollará colelitiasis antes o después de la cirugía (175) y que un $20 \%$ se harán sintomáticas en el seguimiento (176), lo que da indicación a la cirugía de exéresis, y aceptamos que todas serían por laparoscopia y sin complicación, o sea el GRD 494, que en el año 2013 en el HUCA tuvo un coste de 3.126,62€. De los 97 pacientes colecistectomizados, 20 precisarían un ingreso para colecistectomía, lo que supone un coste de 62.532€, que dividido por la muestra nos da un ahorro de $203 €$ por paciente.

\subsubsection{Estudios preoperatorios}

Los estudios se realizaron según protocolo con un porcentaje de cumplimiento alto. El porcentaje de infección por Helicobacter pylori es similar al del resto de la población (177), y un porcentaje de erradicación condicionado probablemente por el aumento de las resistencias a antibióticos (178). El ecocardiograma, con un $90 \%$ de estudios normales en la actualidad nos hace ser más selectivos en su indicación. 


\subsection{Pacientes con BIG}

El BIG es efectivo en la pérdida de peso a corto plazo. La evolución ponderal con la colocación del BIG fue similar a la de otras publicaciones, para IMC mayor de 40 (179), en las que se recoge un PSP del 27\%, frente a nuestro $24 \%$ para IMC más altos. En cuanto a las complicaciones, también similar a resultados recogidos en un meta-análisis (180) donde hay un $4,2 \%$ de retirada anticipada del BIG, frente a nuestro $4,3 \%$, y un $13,6 \%$ de pacientes con vómitos o dolor abdominal, frente a nuestro $15,6 \%$ de complicaciones generales.

\subsection{Cirugía}

El hecho que sea el mismo tipo de tratamiento en todos los pacientes del estudio es un dato a favor a la hora de realizar una evaluación económica. La tendencia actual es a realizar el mayor número posible de intervenciones por laparoscopia, y en el caso de la cirugía bariátrica es el abordaje de elección. Tenemos un 7,6\% de conversión, un porcentaje elevado para lo exigible hoy en día, pero hay que pensar que ya han pasado casi 10 años desde los primeros casos, y sin duda la influencia de la experiencia del equipo quirúrgico, y la mejora del equipamiento, disminuye esta cifra hasta estar por debajo del $3 \%$ (181). La razón de existir diferencias de peso entre el grupo abierto y laparoscópico se explica por la selección de casos menos complejos al inicio de este abordaje, con los casos de IMC más bajo, así como el PSP de los pacientes intervenidos por laparoscopia es mayor, por tratarse de los de menor IMC. De la misma manera que para los tres tipos de bypass se ve modificada la vía de abordaje, en la medida en que el IMC condiciona cada uno de ellos. EI tiempo quirúrgico, mayor en la cirugía laparoscópica, por la dificultad que representan este tipo de cirugías, y la estancia media, mayor en las abiertas, son las características principales de cada uno de los abordajes.

De las complicaciones postoperatorias, destacar la infección de herida quirúrgica (IHQ), que representa el $60 \%$ de las complicaciones quirúrgicas, y afecta a un $15 \%$ de la serie, un porcentaje elevado quizá por un exceso de celo 
a la hora de registrar por nuestra parte cualquier drenaje de herida quirúrgica. Si lo diferenciamos por el tipo de abordaje, y comparamos con los resultados de Hutter (167) en el caso de la neumonía tuvimos para la cirugía abierta un $1,5 \%$ vs $1,3 \%$ y para la laparoscópica un 0,9 vs 0,4 , resultados equiparables teniendo en cuenta la muestra. De la ITU en cirugía abierta hubo un $7,4 \%$ vs $1 \%$, y en laparoscópica $4,5 \%$ vs $0,7 \%$, quizá respondiendo a este mismo fenómeno de exceso de recogida de datos, con orinas patológicas sin trascendencia clínica. De la $\mathrm{IHQ}$, si diferenciamos entre superficial y profunda, para la primera y por abordaje abierto tuvimos un $17,4 \%$ vs $4 \%$, y laparoscópica $9,1 \%$ vs $1,5 \%$, condicionado este último probablemente por la técnica quirúrgica, con la introducción de la sutura mecánica circular a través de uno de los puertos ampliado. La infección profunda para la abierta fue de $1,5 \%$ vs $1,4 \%$ y laparoscópica $0,9 \%$ vs $0,46 \%$, resultados equiparables.

La hemorragia, que debe presentar cifras inferiores al 4\%, es mayor en el grupo de la laparoscopia, influido por el periodo de aprendizaje y evolución del equipo quirúrgico en este aspecto, resultado similar a los observados en otras series (182). La obstrucción fue en su totalidad en la cirugía laparoscópica, por fallo en la técnica quirúrgica, asumimos que por el mismo motivo.

La dehiscencia según series varía del 0 al 4,4\%, si bien los resultados más recientes apuntan que está en torno al 1\% (183), con mayor presencia en la cirugía abierta y precisando reintervención en la mayoría de los casos, resultados similares a los de nuestra serie.

El porcentaje de reintervenciones en cirugía abierta es bajo, del 1,5\%, considerando la referencia en torno al 5\% tanto para abierta como laparoscópica, y del 7,3\% en laparoscópica, alto. No hubo ningún fallecido, y debemos considerar como deseable estar por debajo del $0,5 \%$.

Los ingresos son prolongados, especialmente en cirugía abierta con una estancia media de 13 días frente a los 7,9 de la laparoscópica, más próximos a la media nacional entre los años $2005-2010$ con 6,8 días (9). Pero estas cifras tan prolongadas nos disminuyen el número de reingresos, que en nuestra serie es del 2/307 (0,6\%), y que según la revisión de Hutter puede oscilar del 6 al 9\% para estancias medias de 2,6 a 3,7 días (167). 


\subsection{Seguimiento de la intervención}

\subsubsection{Evolución ponderal}

Si bien insistimos en la importancia de no ganar peso en el periodo antes de la cirugía y motivamos una pérdida de peso durante el tiempo que estén en lista de espera, no imponemos una cifra de pérdida de peso preoperatoria como posible acción de unos mejores resultados en PSP y menor tiempo quirúrgico como ha sido publicado (184), aunque hay autores que no han encontrado estas ventajas (185), cuestionando su importancia. Analizando la serie completa, el peso medio de la primera consulta es de $131,8 \mathrm{~kg}$ y el peso preoperatorio es de $127,3 \mathrm{~kg}$. Sin valorar los pacientes a los que se les colocó un BIG preoperatorio, la media de peso bajó de 127,3 a 125,8 kg, durante el tiempo que están en espera de la cirugía (una media de 12,2 meses), que teniendo en cuenta la evolución natural de la obesidad, nos indica el valor de las recomendaciones realizadas en consulta, y la dieta completa hipocalórica e hiperproteica que se indica la semana previa a la intervención.

En la evolución ponderal postoperatoria encontramos cuatro momentos, en los que queda reflejado que la pérdida de peso más llamativa se produce durante el primer año postoperatorio, manteniéndose o con leve descenso en el año siguiente. Un PSP entorno al 70\% de media y un PEIMCP del 75\% al año son resultados equiparables a estudios de revisión del BPG (84) e incluso superiores a otros (186).

\subsubsection{Comorbilidades}

La evolución de las comorbilidades se ha expuesto junto con la descripción de las mismas en la cohorte.

\subsubsection{Calidad de vida}

La calidad de vida medida mediante el cuestionario específico de cirugía bariátrica BAROS destaca una valoración global de "mucho mejor" respecto a 
antes de la intervención en más del $80 \%$ de los pacientes. Tan solo tenemos un paciente que se encuentra "peor" y otro "mucho peor". Por apartados es en la autoestima y la actividad física donde refieren sentirse con más frecuencia "mucho mejor". No tanto en relaciones sociales ni en el apartado laboral, como puede ser esperable, por no estar tan directamente relacionado con la pérdida de peso y resolución de comorbilidades. En el apartado de relaciones sexuales no hubo cambios en la mitad de ellos.

En pérdida de peso, si entendemos por fracaso un PSP menor del 50\%, con las limitaciones de tiempo existentes de corto plazo, en esta situación tenemos un $11 \%$, una cifra similar a otros trabajos (187).

Para la evaluación final BAROS, en la que se recogen todos los aspectos de la cirugía, debemos diferenciar entre los que presentan comorbilidades al ingreso de los que no, por puntuar sobre diferente escala. Tenemos más de un $80 \%$ con resultado muy bueno o excelente, mejor en el caso de aquellos sin comorbilidades.

En nuestro análisis del BAROS encontramos mejores resultados en pacientes jóvenes, resultado similar a trabajos previos (188), que aunque en aquellos sin comorbilidades no es significativa, aunque la diferencia de edad entre grupos es también de unos 6 años, siendo probablemente más un problema de tamaño muestral (35 pacientes). A diferencia de otros autores (189) no encontramos diferencia en el tipo de abordaje ni en el IMC, si bien en los de BAROS sin comorbilidades podemos ver cierta tendencia para el IMC $(p=0,072)$.

\subsubsection{Pruebas realizadas}

En las pruebas realizadas por protocolo, encontramos un uso adecuado de la gastroscopia y ecografía selectiva en el primer año postoperatorio. No tanto el EGD, con un elevado número de exploraciones normales.

La ecografía revela un $24,5 \%$ de colelitiasis al año de la cirugía, resultado esperable y que responde principalmente a la pérdida de peso acelerada de estos pacientes (190). 


\subsection{Modelos de estimación de costes}

\section{Coste por GRD}

En este punto debemos hacer mención al Benchmarking, la manera que tenemos de compararnos con nuestros "iguales", hospitales de más de 1.000 camas. Así para el año 2013, el GRD 288 (el 90\% de nuestra serie) tiene una estancia media de 5,79 días, una mortalidad de $0,1 \%$ y un coste de $7.055 €$ (191). Nuestra estancia media (DE) para este grupo de GRD es de 8,3 (3) días, que si seleccionamos aquellos intervenidos por laparoscopia, abordaje mayoritario en ese año, la media nos queda en 7,3 (2,3) días, cifra más acorde con la referencia aunque sin llegar a alcanzarla. La mortalidad es baja, en nuestro caso no hay ningún paciente, pero hay que recordar que es el GRD teórico sin complicación. El coste en nuestro hospital (4.631€) es mucho menor que en el resto de hospitales de nuestras características, con una diferencia de coste del $35 \%$, por un menor peso ya que usamos una versión distinta de GRDs, y por tener menor coste de unidad de peso para el GFH Cirugía General con Hospitalización.

Respecto al GRD 565, con 25 casos, la estancia media debería ser de 15 días, estamos en los $22(23,1)$ días. La mortalidad $2,7 \%$ y el coste $12.047 €$ frente a nuestros $13.558 €$, mostrando mayor similitud que en el caso anterior.

Por último el GRD 877 del que tenemos dos casos, la estancia media debería ser de 67 días, tenemos un paciente de 42 días y otro de 407 días. La mortalidad de este grupo es del $31 \%$. El coste de $107.911 €$ frente a nuestros $124.311 €$.

En lo que implica a este coste encontramos diferencia en IMC altos, así como un mayor coste en los que se complicaron y tuvieron un BIG previo, en la medida en que son costes que sí modifican el coste por GRD, y fueron los de abordaje laparoscópico los más baratos, por tener mayor número de GRD 288 en este grupo. 
Si bien es un registro que nos proporciona mucha información de lo que queramos analizar, a la hora de una correcta evaluación económica queda limitado, por lo que nos centraremos en el coste individualizado.

Queda explicado el origen de todos nuestros datos, de los que destaca el precio más elevado del material de la cirugía laparoscópica $(4.442,42 €)$, que es equiparable al coste que interpreta el GRD más frecuente (288) que supone el ingreso $(4.631,6 €)$. El coste principal del ingreso es el derivado del procedimiento quirúrgico.

Son pocos los estudios en obesidad de nuestro país que hagan la evaluación económica del procedimiento quirúrgico sin basarse en los GRDs, pero encontramos un estudio coste-efectividad (131) de varios países europeos en pacientes diabéticos intervenidos mediante BPG en el año 2009, donde se calcula que el coste de la intervención es de 4.000€, de los que la mitad corresponderían a material, mucho menor coste que en nuestro cálculo individualizado. El ingreso completo serían 5.483€, más en el entorno nuestro del cálculo por GRD, que en el individualizado.

La cirugía laparoscópica ya hace muchos años que se muestra como una alternativa coste-efectiva a la cirugía abierta, a pesar de precisar más tiempo de quirófano (más coste), ya que tiene menos estancia y recuperación más rápida, con una pérdida de peso similar y mejor calidad de vida al año (192). En nuestro estudio, con las limitaciones de selección existentes que implican que los pacientes a los que se les realizó un BPG laparoscópico son los de menor IMC, encontramos mejor resultado BAROS en los laparoscópicos, pero con diferencias no significativas.

La laparoscopia se muestra más coste-efectiva, aunque sin llegar a tener significación, si usamos el coste por GRD como método de valoración económica. Por un lado por tener mejor efectividad (en este caso medido por el resultado BAROS), y por otro por tener menor número de GRDs de complicación en este grupo, y ningún GRD 877, que son los que encarecen. Los días de ingreso, menores, y el material que en la cirugía laparoscópica es más caro, no son tenidos en cuenta por con este método, al menos de modo directo. 


\section{Coste por paciente}

Si hacemos uso de la valoración individualizada del coste la media será a favor de la cirugía abierta, que a su vez se muestra más coste-efectiva, aunque igualmente no llega a alcanzar significación. Esto es debido a que el material quirúrgico es más barato y los días de ingreso aunque sean más no superan este menor gasto en material, y la efectividad en forma de BAROS, que a pesar de ser menor en la abierta, tampoco llega a compensar al de la laparoscópica, lo que nos da un mejor resultado CE en la abierta.

En el análisis encontramos un mayor coste para los IMC altos, para estancias prolongadas, para aquellos con complicaciones y con BIG previo, así como para el abordaje laparoscópico, todos ellos esperables en la medida que computan como costes. Nos sorprende que se muestren como más caros los pacientes con SAHS, sin tener significación otras patologías como la DM, pero es cierto que los pacientes con SAHS tienen mayor IMC.

Como en estudios previos (193) la cirugía laparoscópica en nuestra serie presenta menos estancia, más tiempo quirúrgico y mejor pérdida de peso.

\subsection{Evaluación económica}

Para hacer una evaluación económica correcta del tratamiento quirúrgico de la obesidad debemos comenzar por saber cuál es el coste real de la intervención, cantidad muy variable por la diversidad de sistemas sanitarios y su financiación. En EEUU encontramos publicaciones desde los $8.090 \$$ de Snow (132), 17.400 de Sinha (194), 20.948\$ del Medicare (195), hasta los 25.000\$ de Myers (196) y Morton (197) para el BPG laparoscópico. En España se sitúa en los 7.468€ por GRD (9) para la cirugía bariátrica, en nuestro caso es de $4.631 €$ y para el ministerio 7.055€ para el mismo año; por coste individualizado está en los 5.483€ en otros estudios para el BPG laparoscópico (131), en nuestra evaluación algo por encima de los diez mil euros. 
En este sentido, en lo que se refiere al coste del ingreso, queda demostrado lo limitado del método clásico de contabilidad analítica mediante GRD, comportándose de manera más fiable e individualizada el método bottom-up.

Para estudiar el ahorro que pudiera suponer esta cirugía a los dos años, nos hemos centrado en el consumo de fármacos en las patologías más prevalentes, por las características del estudio, pero entran en juego una gran cantidad de costes no controlables (que recaen sobre el paciente y en segundo término sobre la sociedad), en los que dejamos sin evaluar costes indirectos (derivados de la pérdida de días de trabajo y cambios de la capacidad física del paciente; como absentismo laboral, incapacidad permanente,...) y costes intangibles (relacionados con el dolor o sufrimiento del paciente); así como el consumo de recursos hospitalarios o ambulatorios que no tenemos recogidos (consultas a los Especialistas, visitas al médico de Atención Primaria, pruebas en relación con sus pluripatologías,....).

Calculamos las principales comorbilidades por separado, las principales y que mayor gasto sanitario implican son la HTA y la DM, la primera con más prevalencia y la segunda con mejor resolución $(2,135,198)$. El gasto sanitario de la HTA en España en costes directos (199) y el porcentaje que supone la medicación (156), así como los costes directos de la DM (200) y su porcentaje en medicación (157), queda recogido en la tabla 47.

Tabla 47. Gasto sanitario español, costes directos por patología: HTA y DM

\begin{tabular}{|l|c|c|}
\hline & HTA & DM \\
\hline Gasto Sanitario (\%) & $3-4$ & $6-8$ \\
\hline Gasto de medicación (\%) & 70 & 15 \\
\hline Gasto por paciente/año $(€)$ & 1.312 & 1.770 \\
\hline Gasto en fármacos/año $(€)$ & 894 & 265 \\
\hline
\end{tabular}

HTA

La prevalencia de HTA en nuestra serie es del $53 \%$, de los que en torno al $90 \%$ está medicado, con una media de fármacos de 1,33 antes de la intervención, pasando a los dos años al 0,3, y una prevalencia del 12,3\%, resultados similares a Gould et al (201) con una prevalencia inicial del $46 \%$ pasando al $10 \%$ tras cirugía. El ahorro de consumo de fármacos para la HTA a los dos años es de $764 €$ por paciente de la muestra y $1.469 €$ por paciente con HTA. 


\section{DM}

La prevalencia de DM en nuestra serie es del $27 \%$, de los que el $76 \%$ está medicado, con una media de fármacos de 1,14 antes de la intervención, pasando al 0,11 a los dos años, y una prevalencia del 2,9\% en los que a pesar de seguir con la DM presentan mejoría en modo de disminución de la medicación. Resultados similares a Gould et al (201) donde se recoge una disminución de la incidencia del 26 al 4\% tras la cirugía. El ahorro en fármacos para la DM a los 2 años es de $131 €$ por paciente de la muestra y $485 €$ por paciente con DM. El ahorro en fármacos para la muestra completa en el apartado de DM queda muy diluido por su baja prevalencia y por la pequeña proporción de gasto que implican los fármacos en estos pacientes.

\section{DL}

La prevalencia de DL en nuestra serie es del $23 \%$, de los que el $48 \%$ está medicado, con una media de fármacos de 0,52 antes de la intervención, pasando al 0,07 a los dos años, y una prevalencia del 2,6\%. El ahorro en fármacos a los dos años es de 114€ por paciente de la muestra y $500 €$ por paciente con DL.

\section{SAHS}

La prevalencia de esta patología en nuestra serie es de un $58 \%$ de los que el 98\% usan CPAP. El ahorro en el dispositivo a los dos años es de $572 €$ por paciente de la muestra y $1.060 €$ por paciente con SAHS.

\section{Depresión}

Entre las patologías que peor responden a la cirugía se encuentra esta. La prevalencia en nuestra serie es de un $40 \%$, de los que el $81 \%$ está medicado. El ahorro en fármacos a los dos años es de $241 €$ por paciente de la muestra y $716 €$ por paciente con depresión. 
En el resto de patologías no hacemos una estimación de reducción de costes por no disponer de estudios adecuados para estimar el coste de cada una de ellas. La artrosis sin duda representa un elevado coste socio-sanitario, así como otras patologías derivadas de la obesidad, como algunos tipos de cáncer o la patología cardiaca. Tampoco entramos a valorar el coste de la medicación que precisan estos pacientes de manera profiláctica, principalmente suplementos vitamínicos y minerales.

Con todo ello, y teniendo en cuenta las principales patologías, a los dos años tenemos un ahorro de $1.822 €$ por paciente intervenido de nuestra serie, independientemente de las patologías previas que padezca, y de $4.230 €$ en caso de padecer las cinco patologías analizadas. Como en estudios previos (134) se acepta que el BPG tiene beneficio económico en disminución de fármacos y dispositivos médicos, lo que no está claro si es coste-ahorro en fármacos, lo que sí parece claro es que nuestro cálculo está infravalorando el ahorro real, por lo comentado, y que esta disminución de la mediación no se ciñe exclusivamente a los dos años. Podemos añadir en este punto los $203 €$ generados en nuestro cálculo por la colecistectomía profiláctica, con una suma final (4.433€) muy similar al coste que el HUCA interpreta que cuesta este tipo de intervención (4.631€), con el sistema de evaluación económica que maneja (GRD 288), para pacientes con todas las comorbilidades.

Con este cálculo, y asumiendo el coste individualizado como el adecuado, podríamos situar el punto en el que se cubren gastos en torno a los 8 años para la serie en general, similar al estudio de Faria et al (202), en Portugal, donde se hace especial mención a lo necesario de ofrecer este tratamiento lo antes posible, ya que en gente más joven (menores de 40 años), el tratamiento tiene mayor coste-ahorro, afirmación que por nuestros resultados compartimos; y similar también a Nguyen (136) que sitúa este punto en los 7 años, con una reducción al año de 2.016 en fármacos al año de la cirugía, cifra que nuestra serie necesita de dos años para alcanzarla, para la serie completa. Y por los cálculos realizados podemos añadir que en función de las comorbilidades que padezca cada paciente, disminuiría este tiempo. Estas afirmaciones desde la prudencia ya que nuestro estudio es detallado en los primeros dos años, y todo aquello que suceda más allá de este periodo se escapa a este análisis. 
Si tenemos en cuenta nuestro tiempo medio de LEQ (12,2 meses), esto supone un gasto que dejamos de mejorar. Snow et al (132) encuentran que el gasto generado en un periodo preoperatorio de 22 meses excede el coste del procedimiento, que calcula en referencia a un coste del procedimiento laparoscópico de 8.090\$. En nuestro caso dependerá de la medicación de cada paciente para hacer este cálculo, y si tenemos en cuenta el coste del principal GRD (4.631€), un paciente con todas las patologías estudiadas y con el ahorro de la colecistectomía, estaríamos en un coste similar a los 2 años. La media de reducción de fármacos a dos años, en ese estudio, es de 2,7 por paciente.

En el estudio de Lopes et al (135) el descenso es de 2,03 fármacos/paciente. En nuestro estudio partimos de 1,96 fármacos/paciente (602 fármacos) para la serie completa y de 2,86 fármacos/paciente que toma algún tipo de medicación (210 pacientes), y a los 2 años 0,53 fármacos/paciente (164 fármacos) de la serie completa y 0,78 fármacos/ paciente medicado. Una reducción en torno al $70 \%$, similar a los estudios mencionados. En el de Nguyen et al (136) esta reducción es mayor, de 2,4 fármacos de media antes de la cirugía, a 0,2 al año.

Tabla 48. Estudios de amortización de la cirugía bariátrica

\begin{tabular}{|c|c|c|c|c|c|c|}
\hline Autor & Intervención & $\begin{array}{l}\text { Datos } \\
\text { económicos }\end{array}$ & $\begin{array}{l}\text { No } \\
\text { Intervenidos }\end{array}$ & № Controles & $\begin{array}{l}\text { Tiempo } \\
\text { (años) }\end{array}$ & $\begin{array}{l}\text { Recupera } \\
\text { inversión } \\
\text { (años) }\end{array}$ \\
\hline $\begin{array}{l}\text { Sampalis } \\
2004 \\
\text { Canada } \\
(203)\end{array}$ & BPG, GVA & $\begin{array}{l}\text { Seguro. } \\
\text { Costes } \\
\text { directos }\end{array}$ & 1035 & 5746 & 5 & 3,5 \\
\hline $\begin{array}{l}\text { Cremieux } \\
2008 \\
\text { USA (126) }\end{array}$ & $\begin{array}{l}\text { BPG, GVA, } \\
\text { BG }\end{array}$ & $\begin{array}{l}\text { Seguro. } \\
\text { Costes } \\
\text { directos }\end{array}$ & 3651 & 3651 & 4 & $2-4$ \\
\hline $\begin{array}{l}\text { Klein } \\
2010 \\
\text { USA (127) }\end{array}$ & $\begin{array}{l}\text { BPG, GVA, } \\
\text { BG }\end{array}$ & $\begin{array}{l}\text { Seguro. } \\
\text { Costes } \\
\text { directos }\end{array}$ & 808 (DMT2) & 808 (DMT2) & 3 & 2,16 \\
\hline $\begin{array}{l}\text { Zanela } \\
2012 \\
\text { Méjico } \\
(204)\end{array}$ & BPG & $\begin{array}{l}\text { Sanidad } \\
\text { pública. } \\
\text { Costes } \\
\text { directos }\end{array}$ & 150 & 150 & 10 & $\begin{array}{l}6,8 \text { y } 4,4 \\
\text { en DMT2 }\end{array}$ \\
\hline $\begin{array}{l}\text { Weiner } \\
2013 \\
\text { USA (102) }\end{array}$ & $\begin{array}{l}\text { BPG, BG, } \\
\text { Otros } \\
\text { restrictivos }\end{array}$ & Seguro & 29820 & 29820 & 6 & No \\
\hline $\begin{array}{l}\text { Morton } \\
2015 \\
\text { USA (197) }\end{array}$ & $\mathrm{BPG}$ & Seguro & 823 & 786 & 4 & $\begin{array}{l}3 \text { y } 2 \text { en } \\
\text { DMT2 }\end{array}$ \\
\hline
\end{tabular}

BPG: Bypass gástrico. GVA: Gastroplastia vertical anillada. BG: Banda gástrica. DMT2: Diabetes mellitus tipo 2. 
Los pacientes con DM merecen un momento de análisis aparte, sin ser motivo de este estudio, trabajos recientes a largo plazo, con seguimientos de 15 años, apuntan la conveniencia de priorizar a estos pacientes (163), opinión que sin duda compartimos. Como hemos visto en nuestros pacientes el consumo de recursos es mucho mayor en los que padecen DM por la cantidad de enfermedades asociadas, lo que supone un grupo muy sensible a la hora de realizar cualquier evaluación económica. Nos sorprende que no exista diferencia de peso entre los que padecen DM y los que no; y el hecho de que sí exista diferencia de edad, mayores los que padecen DM probablemente por agotamiento de la función endocrina del páncreas, lo que apoya más la idea de priorizar a estos pacientes.

Para finalizar recordar que el paciente obeso consume per se más recursos, con independencia del procedimiento que se le vaya a realizar o motivo de ingreso $(205,206)$. Son pacientes que precisan de una gran cantidad de prestaciones tanto ambulatorias, hospitalarias, como farmacológicas que al ritmo actual, pueden llegar a hacer el sistema sanitario insostenible, representando un importante reto de nuestro tiempo que requiere la colaboración y los esfuerzos de todos (207), y cuanto antes revirtamos su situación, antes los devolveremos a un estado saludable, en el que los costes son menores, así como mejoraremos su cantidad y calidad de vida. 


\section{Limitaciones del estudio.}

En un estudio de costes como este nos vemos limitados por múltiples razones, entre otras por el diseño retrospectivo en el que en la recogida de datos dejamos sin registrar costes tan importantes como los derivados del desempleo, absentismo laboral, incapacidades y exclusión social consecuencia de la obesidad. Todos los gastos más allá de los reflejados en la historia clínica, como por ejemplo el gasto personal, entendido en algunos estudios como el principal gasto de estos pacientes (147). También nos vemos limitados en la recogida de datos de relevancia económica, como los resultantes de las secuelas de la cirugía bariátrica, tanto en tratamiento de eventraciones como de cirugía plástica. No hemos incluido las complicaciones más allá de las que puedan darse durante el ingreso de la cirugía o en los treinta días posteriores al alta, así como todo lo que se realice de manera ambulatoria fuera del protocolo de seguimiento, tanto en consulta de Atención Primaria, como de Especializada. Los cambios en la medicación desde la inclusión en LEQ hasta la cirugía no están recogidos, si bien en los primeros pacientes operados esto pudiera carecer de importancia por la corta demora, en los últimos sí puede representar una limitación, ya que el tiempo en lista de espera tuvo una media de un año, con un tiempo máximo que fue más allá de los dos años. El seguimiento se estableció en los dos años, periodo considerado de corto plazo (77), que es cuando se suele producir la mayor pérdida de peso, pudiendo existir a partir de aquí generalmente distintos grados de reganancia y reaparición de comorbilidades. Si bien no es el objetivo principal del estudio, en el ámbito del análisis de coste-efectividad lo ideal es tener un grupo con el que compararse, que en este caso serían pacientes no intervenidos. Como cualquier intervención tiene una curva de aprendizaje, establecida en nuestro estudio en los treinta primeros pacientes de abierta y laparoscópica, insuficiente para algunos autores (208), en cualquier caso con el aumento de la experiencia los resultados mejoran. Tratándose de un estudio observacional tenemos la limitación de la recogida de datos, aunque en este estudio todos fueron revisados por la misma persona, y recogidos de otras fuentes, como el seguimiento de Endocrinología u otros Servicios, en caso de duda en la recogida de algún dato. Los resultados son de una cohorte de pacientes 
intervenidos mediante bypass gástrico, no siendo los resultados extrapolables o comparables con otras medidas o intervenciones para el tratamiento de la obesidad. El coste de fármacos es estimativo, por la gran cantidad de presentaciones y precios de un mismo principio activo (155), lo que nos hizo desistir del gasto en fármacos individualizado. No incluimos otras patologías con potencial beneficio tras la cirugía, como la prevención de algunos tipos de tumores, patología cardiovascular, desordenes ginecológicos o problemas músculo-esqueléticos. 




\section{CONCLUSIONES}

La cirugía bariátrica resuelve patologías graves y costosas, hasta la fecha irreversibles con otros tratamientos. A los dos años, en pacientes con pluripatologías el ahorro sólo en fármacos podría compensar los gastos inherentes al tratamiento quirúrgico.

Los resultados a los dos años de la cirugía en pérdida de peso, resolución de comorbilidades, calidad de vida y modificación de la medicación están dentro de los estándares de los trabajos publicados de referencia, y son el fundamento clínico de su aplicación.

El coste por proceso mediante GRD se muestra insuficientes para hacer una correcta evaluación económica, recomendamos por tanto un método de estimación de coste por paciente.

Los pacientes que más se benefician de la cirugía bariátrica son los más jóvenes y con IMC más bajo. Por patologías la DM, el SAHS y la HTA son las comorbilidades que mejor van a responder, y la artrosis y la depresión las que peor. Lo que nos refuerza la idea del impulso y realización precoz de esta cirugía.

El análisis coste-efectividad, con las características de nuestra serie, no muestra resultados concluyentes como para afirmar que una vía de abordaje es superior a la otra. 



\section{BIBLIOGRAFÍA}





\section{BIBLIOGRAFÍA}

1. Informe anual del Sistema Nacional de Salud 2012. MINISTERIO DE SANIDAD, SERVICIOS SOCIALES E IGUALDAD; 2015.

2. Buchwald $H$, Avidor $Y$, Braunwald E, Jensen MD, Pories W, Fahrbach $K$, et al. Bariatric surgery: a systematic review and meta-analysis. JAMA. 2004;292(14):1724-37.

3. Buchwald H, Estok R, Fahrbach K, Banel D, Jensen MD, Pories WJ, et al. Weight and type 2 diabetes after bariatric surgery: systematic review and meta-analysis. Am J Med. 2009;122(3):248-56.

4. Buchwald H, Oien DM. Metabolic/bariatric surgery worldwide 2011. Obes Surg. 2013;23(4):427-36.

5. ASMBS. Bariatric Surgery Procedures - ASMBS [Internet]. American Society for Metabolic and Bariatric Surgery. [citado 17 de abril de 2016]. Recuperado a partir de: https://asmbs.org/patients/bariatric-surgery-procedures

6. Stocker DJ. Management of the bariatric surgery patient. Endocrinol Metab Clin North Am. 2003;32(2):437-57.

7. Carrasco F, Klaassen J, Papapietro K, Reyes E, Rodríguez L, Csendes $A$, et al. Propuesta y fundamentos para una norma de manejo quirúrgico del paciente obeso. Rev Médica Chile. 2005;133(6):699-706.

8. Sjöström L, Narbro K, Sjöström CD, Karason K, Larsson B, Wedel H, et al. Effects of bariatric surgery on mortality in Swedish obese subjects. $\mathrm{N}$ Engl J Med. 2007;357(8):741-52.

9. Sánchez-Santos R, Sabench Pereferrer F, Estévez Fernandez S, del Castillo Dejardin D, Vilarrasa N, Frutos Bernal D, et al. ¿Es rentable operar a los obesos mórbidos en tiempos de crisis? Análisis coste-beneficio de la cirugía bariátrica. Cir Esp. 2013;91(8):476-84.

10. McCarthy MI. Genomics, type 2 diabetes, and obesity. N Engl J Med. 2010;363(24):2339-50.

11. El-Sayed Moustafa JS, Froguel P. From obesity genetics to the future of personalized obesity therapy. Nat Rev Endocrinol. 2013;9(7):402-13.

12. Cizza G, Rother KI. Beyond fast food and slow motion: weighty contributors to the obesity epidemic. J Endocrinol Invest. 2012;35(2):236-42. 
13. Keith SW, Redden DT, Katzmarzyk PT, Boggiano MM, Hanlon EC, Benca RM, et al. Putative contributors to the secular increase in obesity: exploring the roads less traveled. Int J Obes. 2006;30(11):1585-94.

14. Klein S, Burke LE, Bray GA, Blair S, Allison DB, Pi-Sunyer X, et al. Clinical implications of obesity with specific focus on cardiovascular disease: a statement for professionals from the American Heart Association Council on Nutrition, Physical Activity, and Metabolism: endorsed by the American College of Cardiology Foundation. Circulation. 2004;110(18):2952-67.

15. Rinella ME. Nonalcoholic fatty liver disease: a systematic review. JAMA. 2015;313(22):2263-73.

16. Pi-Sunyer $X$. The medical risks of obesity. Postgrad Med. 2009;121(6):21-33.

17. Balsiger BM, Murr MM, Poggio JL, Sarr MG. Bariatric surgery. Surgery for weight control in patients with morbid obesity. Med Clin North Am. 2000;84(2):477-89.

18. Neligan PJ, Williams N. Nonsurgical and surgical treatment of obesity. Anesthesiol Clin N Am. 2005;23(3):501-23.

19. Pender JR, Pories WJ. Surgical treatment of obesity. Psychiatr Clin North Am. 2005;28(1):219-34.

20. Brolin RE. Gastric bypass. Surg Clin North Am. 2001;81(5):1077-95.

21. Martin-Rodriguez E, Guillen-Grima F, Martí A, Brugos-Larumbe A. Comorbidity associated with obesity in a large population: The APNA study. Obes Res Clin Pract. 2015;9(5):435-47.

22. Martín-Ramiro JJ, Álvarez-Martín E, Gil-Prieto R. Mortalidad atribuible al exceso de peso en España. Med Clínica. 2014;142(12):526-30.

23. Berrington de Gonzalez A, Hartge P, Cerhan JR, Flint AJ, Hannan L, Maclnnis RJ, et al. Body-mass index and mortality among 1.46 million white adults. N Engl J Med. 2010;363(23):2211-9.

24. Whitlock G, Lewington S, Sherliker P, Clarke R, Emberson J, Halsey J, et al. Body-mass index and cause-specific mortality in 900000 adults: collaborative analyses of 57 prospective studies. Lancet. 2009;373(9669):108396.

25. Gomis R, Artola S, Conthe P, Vidal J, Casamor R, Font B. Prevalencia de diabetes mellitus tipo 2 en pacientes ambulatorios con sobrepeso $u$ obesidad en España. Estudio OBEDIA. Med Clínica. 2014;142(11):485-92. 
26. Medrano MJ, Pastor-Barriuso R, Boix R, Barrio JL del, Damián J, Álvarez $\mathrm{R}$, et al. Riesgo coronario atribuible a los factores de riesgo cardiovascular en población española. Rev Esp Cardiol. 2007;60(12):1250-6.

27. Renehan AG, Soerjomataram I, Tyson M, Egger M, Zwahlen M, Coebergh JW, et al. Incident cancer burden attributable to excess body mass index in 30 European countries. Int J Cancer. 2010;126(3):692-702.

28. Thygesen LC, Grønbaek M, Johansen C, Fuchs CS, Willett WC, Giovannucci E. Prospective weight change and colon cancer risk in male US health professionals. Int J Cancer. 2008;123(5):1160-5.

29. Ng M, Fleming T, Robinson M, Thomson B, Graetz N, Margono C, et al. Global, regional, and national prevalence of overweight and obesity in children and adults during 1980-2013: a systematic analysis for the Global Burden of Disease Study 2013. Lancet. 2014;384(9945):766-81.

30. OMS | Obesidad y sobrepeso [Internet]. WHO. [citado 12 de septiembre de 2015]. Recuperado a partir de: http://www.who.int/mediacentre/factsheets/fs311/es/

31. Gutiérrez-Fisac JL, Guallar-Castillón P, León-Muñoz LM, Graciani A, Banegas JR, Rodríguez-Artalejo F. Prevalence of general and abdominal obesity in the adult population of Spain, 2008-2010: the ENRICA study. Obes Rev. 2012;13(4):388-92.

32. Ministerio de Sanidad. ENSE 2011-2012.pdf [Internet]. 2013 [citado 31 de agosto de 2015]. Recuperado a partir de: http://www.ine.es/prensa/np770.pdf

33. OCDE. Obesity-Update-2014.pdf [Internet]. [citado 31 de agosto de 2015]. Recuperado a partir de: http://www.oecd.org/els/health-systems/ObesityUpdate-2014.pdf

34. Beltrán B, Carranza M, Carreras G, Moreno B, Palacios N,, Tojo R, et al. THAO [Internet]. 2011 [citado 31 de agosto de 2015]. Recuperado a partir de: http://thaoweb.com/es/comunidad_cientifica/obesidad_infantil

35. Sicras-Mainar A, Gil J, Mora T, Ayma J. Prevalencia e impacto económico de la obesidad en adultos durante el periodo 2003-2010. Med Clínica. 2012;139(13):599-600.

36. Powers KA, Rehrig ST, Jones DB. Financial impact of obesity and bariatric surgery. Med Clin North Am. 2007;91(3):321-38. 
37. Joaquín Cuevas Aller. Manual práctico de la historia de los Reyes de León [Internet]. Instituto Cepedano de Cultura; [citado 29 de agosto de 2015]. 83 p. Recuperado a partir de: http://www.casadellibro.com/libro-manualpractico-de-la-historia-de-los-reyes-de-leon/9788493404680/1078132

38. Hopkins KD, Lehmann ED. Successful medical treatment of obesity in 10th century Spain. Lancet. 1995;346(8972):452.

39. Baltasar A. More than 1,000 years ago, Sancho the Fat lost his Kingdom... Obes Surg. 2004;14(8):1138.

40. Bariatrics | Define Bariatrics at Dictionary.com [Internet]. [citado 9 de septiembre de 2015]. Recuperado a partir de: http://dictionary.reference.com/browse/bariatrics

41. Buchwald $H$. The evolution of metabolic/bariatric surgery. Obes Surg. 2014;24(8):1126-35.

42. Sundbom M. Laparoscopic revolution in bariatric surgery. World J Gastroenterol. 2014;20(41):15135-43.

43. Kremen AJ, Linner $\mathrm{JH}$, Nelson $\mathrm{CH}$. An experimental evaluation of the nutritional importance of proximal and distal small intestine. Ann Surg. 1954;140(3):439-48.

44. Griffen WO, Bivins BA, Bell RM. The decline and fall of the jejunoileal bypass. Surg Gynecol Obstet. 1983;157(4):301-8.

45. Mason EE, Ito C. Gastric bypass in obesity. Surg Clin North Am. 1967;47(6):1345-51.

46. Wittgrove A, Clark G, Tremblay L. Laparoscopic Gastric Bypass, Rouxen-Y: Preliminary Report of Five Cases. Obes Surg. 1994;4(4):353-7.

47. van Wezenbeek MR, Smulders JF, de Zoete JPJGM, Luyer MD, van Montfort G, Nienhuijs SW. Long-Term Results of Primary Vertical Banded Gastroplasty. Obes Surg. 2015;25(8):1425-30.

48. Reames BN, Finks JF, Bacal D, Carlin AM, Dimick JB. Changes in bariatric surgery procedure use in Michigan, 2006-2013. JAMA. 2014;312(9):959-61.

49. Nguyen NT, Nguyen B, Gebhart A, Hohmann S. Changes in the makeup of bariatric surgery: a national increase in use of laparoscopic sleeve gastrectomy. J Am Coll Surg. 2013;216(2):252-7.

50. Scopinaro N, Gianetta E, Civalleri D, Bonalumi U, Bachi V. Biliopancreatic bypass for obesity: II. Initial experience in man. $\mathrm{Br} \mathrm{J}$ Surg. 1979;66(9):618-20. 
51. Hess DS, Hess DW. Biliopancreatic diversion with a duodenal switch. Obes Surg. 1998;8(3):267-82.

52. Risstad H, Søvik TT, Engström M, Aasheim ET, Fagerland MW, Olsén MF, et al. Five-year outcomes after laparoscopic gastric bypass and laparoscopic duodenal switch in patients with body mass index of 50 to 60: a randomized clinical trial. JAMA Surg. 2015;150(4):352-61.

53. Khorgami Z, Andalib A, Corcelles R, Aminian A, Brethauer S, Schauer P. Recent National Trends In The Surgical Treatment of Obesity: Sleeve Gastrectomy Dominates. Surg Obes Relat Dis. 2015;11(6):S6-8.

54. Regan JP, Inabnet WB, Gagner M, Pomp A. Early experience with twostage laparoscopic Roux-en-Y gastric bypass as an alternative in the supersuper obese patient. Obes Surg. 2003;13(6):861-4.

55. Lazzati A, Guy-Lachuer R, Delaunay V, Szwarcensztein K, Azoulay D. Bariatric surgery trends in France: 2005-2011. Surg Obes Relat Dis. 2014;10(2):328-34.

56. Vilallonga R, Ruiz de Adana JC. Comment on: «Trends in Bariatric Surgery in Spain in the Twenty-First Century: Baseline Results and 1-Month Follow Up of the RICIBA, a National Registry» by Lecube A, et al. Obes Surg. 2016;

57. Vilallonga R, Himpens J, Bosch B, van de Vrande S, Bafort J. Role of Percutaneous Glue Treatment After Persisting Leak After Laparoscopic Sleeve Gastrectomy. Obes Surg. 2015;1-6.

58. Zellmer JD, Mathiason MA, Kallies KJ, Kothari SN. Is laparoscopic sleeve gastrectomy a lower risk bariatric procedure compared with laparoscopic Roux-en-Y gastric bypass? A meta-analysis. Am J Surg. 2014;208(6):903-910910.

59. Zhang C, Yuan Y, Qiu C, Zhang W. A meta-analysis of 2-year effect after surgery: laparoscopic Roux-en-Y gastric bypass versus laparoscopic sleeve gastrectomy for morbid obesity and diabetes mellitus. Obes Surg. 2014;24(9):1528-35.

60. Wang MC, Guo XH, Zhang YW, Zhang YL, Zhang HH, Zhang YC. Laparoscopic Roux-en-Y gastric bypass versus sleeve gastrectomy for obese patients with Type 2 diabetes: a meta-analysis of randomized controlled trials. Am Surg. 2015;81(2):166-71.

61. Golomb I, Ben David M, Glass A, Kolitz T, Keidar A. Long-term Metabolic Effects of Laparoscopic Sleeve Gastrectomy. JAMA Surg. 2015;150(11):10517. 
62. Zhang Y, Wang J, Ju W, Sun X, Cao Z, Cao Z, et al. Laparoscopic sleeve gastrectomy versus laparoscopic Roux-en-Y gastric bypass for morbid obesity and related comorbidities: a meta-analysis of 21 studies. Obes Surg. 2015;25(1):19-26.

63. Lee W-J, Pok E-H, Almulaifi A, Tsou JJ, Ser K-H, Lee Y-C. Medium-Term Results of Laparoscopic Sleeve Gastrectomy: a Matched Comparison with Gastric Bypass. Obes Surg. 2015;25(8):1431-8.

64. Schneck A-S, Stenard F, lannelli A. Back to Sleeve: an Extreme Solution for Specific Complications of the Roux-en-Y Gastric Bypass. Obes Surg. 2015;25(8):1499-501.

65. Nelson DW, Blair KS, Martin MJ. Analysis of obesity-related outcomes and bariatric failure rates with the duodenal switch vs gastric bypass for morbid obesity. Arch Surg Chic III 1960. 2012;147(9):847-54.

66. Dogan K, Gadiot RPM, Aarts EO, Betzel B, van Laarhoven CJHM, Biter LU, et al. Effectiveness and Safety of Sleeve Gastrectomy, Gastric Bypass, and Adjustable Gastric Banding in Morbidly Obese Patients: a Multicenter, Retrospective, Matched Cohort Study. Obes Surg. 2015;25(7):1110-8.

67. Suter M, Donadini A, Romy S, Demartines N, Giusti V. Laparoscopic Roux-en-Y gastric bypass: significant long-term weight loss, improvement of obesity-related comorbidities and quality of life. Ann Surg. 2011;254(2):267-73.

68. Adams TD, Davidson LE, Litwin SE, Kolotkin RL, LaMonte MJ, Pendleton RC, et al. Health benefits of gastric bypass surgery after 6 years. JAMA. 2012;308(11):1122-31.

69. Puche RC. El índice de masa corporal y los razonamientos de un astrónomo. Med B Aires. 2005;65(4):361-5.

70. Renquist K. Obesity classification. Obes Surg. 1998;8(4):480.

71. Salas-Salvadó J, Rubio MA, Barbany M, Moreno B, Grupo Colaborativo de la SEEDO. Consenso SEEDO 2007 para la evaluación del sobrepeso y la obesidad y el establecimiento de criterios de intervención terapéutica. Med Clínica. 2007;128(5):184-96.

72. Gastrointestinal surgery for severe obesity: National Institutes of Health Consensus Development Conference Statement. Am J Clin Nutr. 1992;55(2 Suppl):615S-619S. 
73. Mechanick JI, Youdim A, Jones DB, Garvey WT, Hurley DL, McMahon $\mathrm{MM}$, et al. Clinical practice guidelines for the perioperative nutritional, metabolic, and nonsurgical support of the bariatric surgery patient--2013 update: cosponsored by American Association of Clinical Endocrinologists, The Obesity Society, and American Society for Metabolic \& Bariatric Surgery. Obes Silver Spring. 2013;21 Suppl 1:S1-27.

74. Hsu WC, Araneta MRG, Kanaya AM, Chiang JL, Fujimoto W. BMI cut points to identify at-risk Asian Americans for type 2 diabetes screening. Diabetes Care. 2015;38(1):150-8.

75. Coleman KJ, Huang Y-C, Hendee F, Watson HL, Casillas RA, Brookey J. Three-year weight outcomes from a bariatric surgery registry in a large integrated healthcare system. Surg Obes Relat Dis. 2014;10(3):396-403.

76. Oria HE, Moorehead MK. Bariatric analysis and reporting outcome system (BAROS). Obes Surg. 1998;8(5):487-99.

77. Brethauer SA, Kim J, el Chaar M, Papasavas P, Eisenberg D, Rogers A, et al. Standardized outcomes reporting in metabolic and bariatric surgery. Surg Obes Relat Dis. 2015;11(3):489-506.

78. Deitel M, Greenstein RJ. Recommendations for reporting weight loss. Obes Surg. 2003;13(2):159-60.

79. Cigaina V. Gastric pacing as therapy for morbid obesity: preliminary results. Obes Surg. 2002;12 Suppl 1:12S-16S.

80. Deitel M, Gawdat K, Melissas J. Reporting weight loss 2007. Obes Surg. 2007;17(5):565-8.

81. van de Laar AWJM, Acherman YIZ. Weight loss percentile charts of large representative series: a benchmark defining sufficient weight loss challenging current criteria for success of bariatric surgery. Obes Surg. 2014;24(5):727-34.

82. Rubio MA, Martínez C, Vidal O, Larrad A, Salas J, Pujol J, et al. Documento de consenso sobre cirugía bariátrica. Rev Esp Obes. 2004;(4):22349.

83. Buchwald H, Estok R, Fahrbach K, Banel D, Sledge I. Trends in mortality in bariatric surgery: a systematic review and meta-analysis. Surgery. 2007;142(4):621-632-635.

84. Chang S-H, Stoll CRT, Song J, Varela JE, Eagon CJ, Colditz GA. The effectiveness and risks of bariatric surgery: an updated systematic review and meta-analysis, 2003-2012. JAMA Surg. 2014;149(3):275-87. 
85. Flum DR, Belle SH, King WC, Wahed AS, Berk P, Chapman W, et al. Perioperative safety in the longitudinal assessment of bariatric surgery. $\mathrm{N}$ Engl J Med. 2009;361(5):445-54.

86. Alastrué A, Moreno $\mathrm{P}$, Martínez B. Estado actual del tratamiento quirúrgico de la obesidad móbida. Med Clínica. 2002;119(4):140-4.

87. Burguera B, Ruiz de Adana JC. Repercusiones a largo plazo de la cirugía bariátrica. Cir Esp. 2012;90(5):275-6.

88. Ochner CN, Gibson C, Shanik M, Goel V, Geliebter A. Changes in neurohormonal gut peptides following bariatric surgery. Int $\mathrm{J}$ Obes. 2011;35(2):153-66.

89. Bloomston M, Zervos EE, Camps MA, Goode SE, Rosemurgy AS. Outcome following bariatric surgery in super versus morbidly obese patients: does weight matter? Obes Surg. 1997;7(5):414-9.

90. Orci L, Chilcott M, Huber O. Short versus long Roux-limb length in Rouxen-Y gastric bypass surgery for the treatment of morbid and super obesity: a systematic review of the literature. Obes Surg. 2011;21(6):797-804.

91. Puzziferri N, Roshek TB, Mayo HG, Gallagher R, Belle SH, Livingston $\mathrm{EH}$. Long-term follow-up after bariatric surgery: a systematic review. JAMA. 2014;312(9):934-42.

92. Afshar S, Kelly SB, Seymour K, Lara J, Woodcock S, Mathers JC. The effects of bariatric surgery on colorectal cancer risk: systematic review and meta-analysis. Obes Surg. 2014;24(10):1793-9.

93. Casagrande DS, Rosa DD, Umpierre D, Sarmento RA, Rodrigues CG, Schaan BD. Incidence of cancer following bariatric surgery: systematic review and meta-analysis. Obes Surg. 2014;24(9):1499-509.

94. Sjöström L, Gummesson A, Sjöström CD, Narbro K, Peltonen M, Wedel $\mathrm{H}$, et al. Effects of bariatric surgery on cancer incidence in obese patients in Sweden (Swedish Obese Subjects Study): a prospective, controlled intervention trial. Lancet Oncol. 2009;10(7):653-62.

95. Adams TD, Gress RE, Smith SC, Halverson RC, Simper SC, Rosamond WD, et al. Long-term mortality after gastric bypass surgery. $\mathrm{N}$ Engl $\mathrm{J}$ Med. 2007;357(8):753-61.

96. Sjöström L, Peltonen M, Jacobson P, Sjöström CD, Karason K, Wedel H, et al. Bariatric surgery and long-term cardiovascular events. JAMA. 2012;307(1):56-65. 
97. Fontaine KR, Redden DT, Wang C, Westfall AO, Allison DB. Years of life lost due to obesity. JAMA. 2003;289(2):187-93.

98. Maciejewski ML, Livingston EH, Smith VA, Kavee AL, Kahwati LC, Henderson WG, et al. Survival among high-risk patients after bariatric surgery. JAMA. 2011;305(23):2419-26.

99. Larrad A, Sánchez-Cabezudo C. Indicadores de calidad en cirugía bariátrica y criterios de éxito a largo plazo. Cir Esp. 2004;75(3):301-4.

100. Andreyeva T, Luedicke J, Wang YC. State-level estimates of obesityattributable costs of absenteeism. J Occup Environ Med. 2014;56(11):1120-7.

101. Maciejewski ML, Livingston EH, Smith VA, Kahwati LC, Henderson WG, Arterburn DE. Health expenditures among high-risk patients after gastric bypass and matched controls. Arch Surg Chic III 1960. 2012;147(7):633-40.

102. Weiner JP, Goodwin SM, Chang H-Y, Bolen SD, Richards TM, Johns RA, et al. Impact of bariatric surgery on health care costs of obese persons: a 6year follow-up of surgical and comparison cohorts using health plan data. JAMA Surg. 2013;148(6):555-62.

103. Livingston $\mathrm{EH}$. Is bariatric surgery worth it?: Comment on «Impact of bariatric surgery on health care costs of obese persons». JAMA Surg. 2013;148(6):562.

104. Goldfine $A B$, Vernon $A$, Zinner $M$. Where are the health care cost savings with bariatric surgery in obesity management? JAMA Surg. 2014;149(1):5-6.

105. Banka G, Woodard G, Hernandez-Boussard T, Morton JM. Laparoscopic vs open gastric bypass surgery: differences in patient demographics, safety, and outcomes. Arch Surg Chic III 1960. 2012;147(6):550-6.

106. Weller WE, Rosati C. Comparing outcomes of laparoscopic versus open bariatric surgery. Ann Surg. 2008;248(1):10-5.

107. Nguyen NT, Masoomi H, Magno CP, Nguyen X-MT, Laugenour K, Lane J. Trends in use of bariatric surgery, 2003-2008. J Am Coll Surg. $2011 ; 213(2): 261-6$.

108. Pok E-H, Lee W-J. Gastrointestinal metabolic surgery for the treatment of type 2 diabetes mellitus. World J Gastroenterol. 2014;20(39):14315-28.

109. Hofsø D, Birkeland KI, Holst JJ, Bollerslev J, Sandbu R, Røislien J, et al. Gastric bypass surgery has a weight-loss independent effect on post-challenge serum glucose levels. Diabetol Metab Syndr. 2015;7:69-72. 
110. Salinari S, Bertuzzi A, Guidone C, Previti E, Rubino F, Mingrone G. Insulin sensitivity and secretion changes after gastric bypass in normotolerant and diabetic obese subjects. Ann Surg. 2013;257(3):462-8.

111. Rubino F, Forgione A, Cummings DE, Vix M, Gnuli D, Mingrone G, et al. The mechanism of diabetes control after gastrointestinal bypass surgery reveals a role of the proximal small intestine in the pathophysiology of type 2 diabetes. Ann Surg. 2006;244(5):741-9.

112. Melissas J, Leventi A, Klinaki I, Perisinakis K, Koukouraki S, de Bree E, et al. Alterations of global gastrointestinal motility after sleeve gastrectomy: a prospective study. Ann Surg. 2013;258(6):976-82.

113. Dixon JB, Zimmet P, Alberti KG, Rubino F, International Diabetes Federation Taskforce on Epidemiology and Prevention. Bariatric surgery: an IDF statement for obese Type 2 diabetes. Arq Bras Endocrinol Metabol. $2011 ; 55(6): 367-82$.

114. Rubio MA, Monereo S, Lecube A, Resa J, Masdevall C, de la Cruz Vigo $F$, et al. Joint Position Statement of the SEEN-SECO-SEEDO-SED Societies on metabolic surgery for type 2 diabetes mellitus". Endocrinol Nutr. 2013;60(10):547-8.

115. Bohigas L. La empresa hospitalaria [Internet]. IESE; 1985 [citado 6 de diciembre de 2015]. Recuperado a partir de: http://www.ieseinsight.com/fichaMaterial.aspx?pk=1405\&idi=1\&origen=3\&idiom $\mathrm{a}=1$

116. Urrutia De Hoyos, Ignacio. Implantación de un Modelo de Costes ABC en Hospitales [Internet]. Comunidad de Madrid; 2001 [citado 6 de diciembre de 2015]. Recuperado a partir de: http://www.todostuslibros.com/libros/implantacion-de-un-modelo-de-costes-abcen-hospitales_978-84-451-2202-0

117. Puig-Junoy J, Pinto-Prades JL, Ortún-Rubio V. El análisis coste-beneficio en sanidad. Aten Primaria. 2001;27(6):422-7.

118. Martin LF, Tan TL, Horn JR, Bixler EO, Kauffman GL, Becker DA, et al. Comparison of the costs associated with medical and surgical treatment of obesity. Surgery. 1995;118(4):599-606-607.

119. Borisenko O, Colpan Z, Dillemans B, Funch-Jensen P, Hedenbro J, Ahmed AR. Clinical Indications, Utilization, and Funding of Bariatric Surgery in Europe. Obes Surg. 2015;25(8):1408-16. 
120. Clegg A, Colquitt J, Sidhu M, Royle P, Walker A. Clinical and cost effectiveness of surgery for morbid obesity: a systematic review and economic evaluation. Int J Obes. 2003;27(10):1167-77.

121. Rodicio Miravalles JL, Ciga Lozano MA, González González JJ. Evaluación económica del tratamiento médico frente al quirúrgico en la colecistitis aguda. Gest Eval Costes Sanit. 2007;8(4):29-39.

122. Isabel Gallardo. SEEDO y SECO piden mejorar el acceso a cirugía bariátrica en obesidad grave. Diario Médico [Internet]. 2016 [citado 11 de febrero de 2016]; Recuperado a partir de: http://www.diariomedico.com/2016/01/20/area-profesional/entorno/seedo-yseco-piden-que-se-mejore-el-acceso-a-cirugia-bariatrica-en-obesidad-grave

123. Paxton JH, Matthews JB. The cost effectiveness of laparoscopic versus open gastric bypass surgery. Obes Surg. 2005;15(1):24-34.

124. Picot J, Jones J, Colquitt JL, Gospodarevskaya E, Loveman E, Baxter L, et al. The clinical effectiveness and cost-effectiveness of bariatric (weight loss) surgery for obesity: a systematic review and economic evaluation. Health Technol Assess. 2009;13(41):1-190, 215-357.

125. Craig BM, Tseng DS. Cost-effectiveness of gastric bypass for severe obesity. Am J Med. 2002;113(6):491-8.

126. Cremieux P-Y, Buchwald H, Shikora SA, Ghosh A, Yang HE, Buessing M. A study on the economic impact of bariatric surgery. Am J Manag Care. 2008;14(9):589-96.

127. Klein S, Ghosh A, Cremieux PY, Eapen S, McGavock TJ. Economic impact of the clinical benefits of bariatric surgery in diabetes patients with BMI $\geq 35 \mathrm{~kg} / \mathrm{m}^{2}$. Obesity. 2011;19(3):581-7.

128. Ikramuddin S, Klingman D, Swan T, Minshall ME. Cost-effectiveness of Roux-en-Y gastric bypass in type 2 diabetes patients. Am $\mathrm{J}$ Manag Care. 2009;15(9):607-15.

129. Hoerger TJ, Zhang P, Segel JE, Kahn HS, Barker LE, Couper S. Costeffectiveness of bariatric surgery for severely obese adults with diabetes. Diabetes Care. 2010;33(9):1933-9.

130. Borisenko O, Adam D, Funch-Jensen P, Ahmed AR, Zhang R, Colpan Z, et al. Bariatric Surgery can Lead to Net Cost Savings to Health Care Systems: Results from a Comprehensive European Decision Analytic Model. Obes Surg. 2015;25(9):1559-68. 
131. Anselmino $M$, Bammer $T$, Fernández Cebrián JM, Daoud $F$, Romagnoli $G$, Torres A. Cost-effectiveness and budget impact of obesity surgery in patients with type 2 diabetes in three European countries(II). Obes Surg. 2009;19(11):1542-9.

132. Snow LL, Weinstein LS, Hannon JK, Lane DR, Ringold FG, Hansen PA, et al. The effect of Roux-en-Y gastric bypass on prescription drug costs. Obes Surg. 2004;14(8):1031-5.

133. Narbro K, Agren G, Jonsson E, Näslund I, Sjöström L, Peltonen M, et al. Pharmaceutical costs in obese individuals: comparison with a randomly selected population sample and long-term changes after conventional and surgical treatment: the SOS intervention study. Arch Intern Med. 2002;162(18):2061-9.

134. Gesquiere I, Aron-Wisnewsky J, Foulon V, Haggege S, Van der Schueren B, Augustijns $P$, et al. Medication cost is significantly reduced after Roux-en-Y gastric bypass in obese patients. Obes Surg. 2014;24(11):1896-903.

135. Lopes EC, Heineck I, Athaydes G, Meinhardt NG, Souto KEP, Stein AT. Is Bariatric Surgery Effective in Reducing Comorbidities and Drug Costs? A Systematic Review and Meta-Analysis. Obes Surg. 2015;25(9):1741-9.

136. Nguyen NT, Varela JE, Sabio A, Naim J, Stamos M, Wilson SE. Reduction in prescription medication costs after laparoscopic gastric bypass. Am Surg. 2006;72(10):853-6.

137. Christou NV, Efthimiou E. Bariatric surgery waiting times in Canada. Can J Surg J Can Chir. 2009;52(3):229-34.

138. Gregory DM, Temple Newhook J, Twells LK. Patients' perceptions of waiting for bariatric surgery: a qualitative study. Int J Equity Health. 2013;12:86.

139. Padwal R, Klarenbach S, Wiebe N, Hazel M, Birch D, Karmali S, et al. Bariatric Surgery: A Systematic Review of the Clinical and Economic Evidence. J Gen Intern Med. 2011;26(10):1183-94.

140. Gutiérrez MA, Jiménez de Aberasturi A, Asua J. Guía de Evaluación Económica en el Sector Sanitario. Vitoria: Gobierno Vasco. Departamento de Sanidad; 1999.

141. Vidorreta J, Vázquez-Dodero JC. Sistemas de medición de actividad en los hospitales [Internet]. 1995 [citado 31 de agosto de 2015]. Recuperado a partir de: http://www.iesep.com/es/sistemas-de-medicion-de-actividad-en-loshospitales-3202.html

142. Muñoz E, Tinker MA, Margolis I, Wise L. Surgonomics: the cost of cholecystectomy. Surgery. 1984;96(4):642-7. 
143. Jiménez MR, Romeo Martínez JM, Vázquez Echevarri J, Lacasa MD, Martínez Veiga JL. Comparación entre el coste por proceso y coste por paciente en un servicio de cirugía general. Mapfre Med. 1999;(1):31-6.

144. GRD-ethicon [Internet]. [citado 12 de septiembre de 2015]. Recuperado a partir de: http://www.ethicon.com/sites/default/files/manageddocuments/028217-150121_reimb_bariatric_ca.pdf

145. GRD-covidien [Internet]. [citado 12 de septiembre de 2015]. Recuperado a partir de: http://www.covidien.com/imageServer.aspx/doc336875.pdf?contentID=76481\&c ontenttype=application/pdf

146. Lillo Fernández de Cuevas JM, Rodríguez Blas MC. Estadística de Gasto Sanitario Público 2013. MSSSI; 2015.

147. Roche. Estudio Prospectivo Delphi. Costes sociales y económicos de la obesidad y sus patologías asociadas (hipertensión, hiperlipemias y diabetes). Los tratamientos de futuro y el coste-beneficio generado por su aplicación en el horizonte del año 2005 en España. Madrid: NILO; 1999.

148. von Hippel PT, Bradbury WK. The effects of school physical education grants on obesity, fitness, and academic achievement. Prev Med. 2015;78:4451.

149. OCDE. Obesity-Update-2014-MEXICO_ES.pdf [Internet]. [citado 31 de agosto de 2015]. Recuperado a partir de: http://www.oecd.org/health/healthsystems/Obesity-Update-2014-MEXICO_ES.pdf

150. INE. Contabilidad Nacional Trimestral de España. Tercer trimestre de 2013. 2013.

151. Instituto Nacional de Estadística. Población en Asturias [Internet]. [citado 13 de febrero de 2016]. Recuperado a partir de: http://www.ine.es/jaxiT3/Datos.htm?t=2886

152. Grundy SM, Cleeman JI, Merz CNB, Brewer HB, Clark LT, Hunninghake $\mathrm{DB}$, et al. Implications of recent clinical trials for the National Cholesterol Education Program Adult Treatment Panel III guidelines. Arterioscler Thromb Vasc Biol. 2004;24(8):e149-161.

153. Tarantino I, Warschkow R, Steffen T, Bisang P, Schultes B, Thurnheer $M$. Is routine cholecystectomy justified in severely obese patients undergoing a laparoscopic Roux-en-Y gastric bypass procedure? A comparative cohort study. Obes Surg. 2011;21(12):1870-8.

154. Dindo D, Demartines N, Clavien P-A. Classification of Surgical Complications. Ann Surg. 2004;240(2):205-13. 
155. MSSSI. Ministerio de Sanidad, Servicios Sociales e Igualdad Profesionales de la Salud - Nomenclator [Internet]. [citado 14 de septiembre de 2015]. Recuperado a partir de: http://www.msssi.gob.es/profesionales/nomenclator.do

156. Sicras-Mainar A, Navarro-Artieda R. Coste de la hipertensión arterial según grados de morbilidad en atención primaria. Med Clínica. 2009;133(8):290-5.

157. Crespo C, Brosa M, Soria-Juan A, López-Alba A, López-Martínez N, Soria B. Costes directos de la diabetes mellitus y de sus complicaciones en España (Estudio SECCAID: Spain estimated cost Ciberdem-Cabimer in Diabetes). Av En Diabetol. 2013;29(6):182-9.

158. Oliva J, López-Bastida J, Moreno SG, Mata P, Alonso R. Análisis costeefectividad de un programa de cribado genético en familiares directos de pacientes con hipercolesterolemia familiar en España. Rev Esp Cardiol. 2009;62(1):57-65.

159. FENIN (Federación Española de Empresas de Tecnología Sanitaria). Estudio sobre la eficiencia y los beneficios de las terapias respiratorias domiciliarias [Internet]. Comuniland SL; 2011 [citado 23 de enero de 2016]. Recuperado a partir de: http://www.esteveteijin.com/filesupload/contenido_subapartado/22_34_materialde-descarga_contenido_subapartado.pdf

160. Sicras-Mainar, Antoni, Blanca-Tamayo, Milagrosa, Gutiérrez-Nicuesa, Laura, Salvatella-Pasant, Jordi, Navarro-Artieda, Ruth. Impacto de la morbilidad, uso de recursos y costes en el mantenimiento de la remisión de la depresión mayor en España: estudio longitudinal de ámbito poblacional. Gac Sanit. 2010;24(1):13-9.

161. R: The R Project for Statistical Computing [Internet]. [citado 25 de abril de 2016]. Recuperado a partir de: https://www.r-project.org/

162. Lee CJ, Clark JM, Asamoah V, Schweitzer M, Magnuson T, Lazo M. Prevalence and characteristics of individuals without diabetes and hypertension who underwent bariatric surgery: lessons learned about metabolically healthy obese. Surg Obes Relat Dis. 2015;11(1):142-6.

163. Keating C, Neovius M, Sjöholm K, Peltonen M, Narbro K, Eriksson JK, et al. Health-care costs over 15 years after bariatric surgery for patients with different baseline glucose status: results from the Swedish Obese Subjects study. Lancet Diabetes Endocrinol. 2015;3(11):855-65. 
164. Schauer PR, Kashyap SR, Wolski K, Brethauer SA, Kirwan JP, Pothier $\mathrm{CE}$, et al. Bariatric surgery versus intensive medical therapy in obese patients with diabetes. N Engl J Med. 2012;366(17):1567-76.

165. Brug J. The European charter for counteracting obesity: A late but important step towards action. Observations on the WHO-Europe ministerial conference, Istanbul, November 15-17, 2006. Int J Behav Nutr Phys Act. $2007 ; 4: 11$.

166. Shikora SA. Henry Buchwald, M.D., Ph.D. Obes Surg. 2016;26(1):3-4.

167. Hutter MM, Schirmer BD, Jones DB, Ko CY, Cohen ME, Merkow RP, et al. First Report from the American College of Surgeons -- Bariatric Surgery Center Network: Laparoscopic Sleeve Gastrectomy has Morbidity and Effectiveness Positioned Between the Band and the Bypass. Ann Surg. $2011 ; 254(3): 410-22$.

168. García Ruiz AJ, Divisón Garrote JA, García-Agua Soler N, Morata García de la Puerta F, Montesinos Gálvez AC, Ávila Lachica L. Análisis coste-eficacia de los antihipertensivos en dosis fijas. SEMERGEN - Med Fam. 2013;39(2):7784.

169. Jönsson B, CODE-2 Advisory Board. Revealing the cost of Type II diabetes in Europe. Diabetologia. 2002;45(7):S5-12.

170. SEMERGEN. Artrosis. 2015.

171. Segal JB, Clark JM, Shore AD, Dominici F, Magnuson T, Richards TM, et al. Prompt reduction in use of medications for comorbid conditions after bariatric surgery. Obes Surg. 2009;19(12):1646-56.

172. Stinton LM, Shaffer EA. Epidemiology of gallbladder disease: cholelithiasis and cancer. Gut Liver. 2012;6(2):172-87.

173. Radmard AR, Merat S, Kooraki S, Ashraf M, Keshtkar A, Sharafkhah M, et al. Gallstone disease and obesity: a population-based study on abdominal fat distribution and gender differences. Ann Hepatol. 2015;14(5):702-9.

174. Attili AF, De Santis A, Capri R, Repice AM, Maselli S. The natural history of gallstones: the GREPCO experience. The GREPCO Group. Hepatology. $1995 ; 21(3): 655-60$.

175. Amstutz S, Michel J-M, Kopp S, Egger B. Potential Benefits of Prophylactic Cholecystectomy in Patients Undergoing Bariatric Bypass Surgery. Obes Surg. 2015;25(11):2054-60. 
176. Festi D, Reggiani MLB, Attili AF, Loria P, Pazzi P, Scaioli E, et al. Natural history of gallstone disease: Expectant management or active treatment? Results from a population-based cohort study. J Gastroenterol Hepatol. 2010;25(4):719-24.

177. Baena Díez JM, García Lareo M, Martí Fernández J, León Marín I, Muñiz Llama D, Teruel Gila J, et al. Prevalencia de la infección por Helicobacter pylori en atención primaria: estudio seroepidemiológico. Aten Primaria. 2002;29(9):553-7.

178. López-Góngora S, Puig I, Calvet X, Villoria A, Baylina M, Muñoz N, et al. Systematic review and meta-analysis: susceptibility-guided versus empirical antibiotic treatment for Helicobacter pylori infection. J Antimicrob Chemother. 2015;70(9):2447-55.

179. Tai C-M, Lin H-Y, Yen Y-C, Huang C-K, Hsu W-L, Huang Y-W, et al. Effectiveness of intragastric balloon treatment for obese patients: one-year follow-up after balloon removal. Obes Surg. 2013;23(12):2068-74.

180. Imaz I, Martínez-Cervell C, García-Alvarez EE, Sendra-Gutiérrez JM, González-Enríquez J. Safety and effectiveness of the intragastric balloon for obesity. A meta-analysis. Obes Surg. 2008;18(7):841-6.

181. Podnos YD, Jimenez JC, Wilson SE, Stevens CM, Nguyen NT. Complications after laparoscopic gastric bypass: a review of 3464 cases. Arch Surg Chic III 1960. 2003;138(9):957-61.

182. Hutter MM, Randall S, Khuri SF, Henderson WG, Abbott WM, Warshaw AL. Laparoscopic Versus Open Gastric Bypass for Morbid Obesity. Ann Surg. 2006;243(5):657-66.

183. Smith MD, Adeniji A, Wahed AS, Patterson E, Chapman W, Courcoulas $A P$, et al. Technical factors associated with anastomotic leak after Roux-en- $Y$ gastric bypass. Surg Obes Relat Dis. 2015;11(2):313-20.

184. Alvarado R, Alami RS, Hsu G, Safadi BY, Sanchez BR, Morton JM, et al. The impact of preoperative weight loss in patients undergoing laparoscopic Roux-en-Y gastric bypass. Obes Surg. 2005;15(9):1282-6.

185. Conaty EA, Bonamici NJ, Gitelis ME, Johnson BJ, DeAsis F, Carbray JM, et al. Efficacy of a Required Preoperative Weight Loss Program for Patients Undergoing Bariatric Surgery. J Gastrointest Surg. 2016;20(4):667-73.

186. Bhandari M, Bhandari S, Mishra A, Mathur W, Dixit A. Comparison Between Banded and Nonbanded Roux-En-Y Gastric Bypass with 2-Year Follow-Up: a Preliminary Retrospective Analysis. Obes Surg. 2015;26(1):213-8. 
187. Corcelles R, Boules M, Froylich D, Hag A, Daigle CR, Aminian A, et al. Total Weight Loss as the Outcome Measure of Choice After Roux-en-Y Gastric Bypass. Obes Surg. 2016;1-5.

188. García Díaz E, Martín Folgueras T. Determinantes preoperatorios de resultados del bypass gástrico laparoscópico en el tratamiento de la obesidad mórbida. Nutr Hosp. 2011;26(4):851-5.

189. Al Harakeh AB, Larson CJ, Mathiason MA, Kallies KJ, Kothari SN. BAROS results in 700 patients after laparoscopic Roux-en-Y gastric bypass with subset analysis of age, gender, and initial body mass index. Surg Obes Relat Dis. 2011;7(1):94-8.

190. Coupaye M, Castel B, Sami O, Tuyeras G, Msika S, Ledoux S. Comparison of the incidence of cholelithiasis after sleeve gastrectomy and Roux-en-Y gastric bypass in obese patients: a prospective study. Surg Obes Relat Dis. 2015;11(4):779-84.

191. MSSSI. Registro de Altas de los Hospitales Generales del Sistema Nacional de Salud. CMBD. Norma Estatal. MSSSI [Internet]. [citado 2 de marzo de 2016]. Recuperado a partir de: http://www.msssi.gob.es/estadEstudios/estadisticas/cmbd.htm

192. Nguyen NT, Goldman C, Rosenquist CJ, Arango A, Cole CJ, Lee SJ, et al. Laparoscopic versus open gastric bypass: a randomized study of outcomes, quality of life, and costs. Ann Surg. 2001;234(3):279-289-291.

193. Courcoulas A, Perry Y, Buenaventura P, Luketich J. Comparing the outcomes after laparoscopic versus open gastric bypass: a matched paired analysis. Obes Surg. 2003;13(3):341-6.

194. Sinha AC, Singh PM, Bhat S. Are we operating too late? Mortality Analysis and Stochastic Simulation of Costs Associated with Bariatric Surgery: Reconsidering the BMI Threshold. Obes Surg. 2015;26(1):219-28.

195. Flum DR, Kwon S, MacLeod K, Wang B, Alfonso-Cristancho R, Garrison LP, et al. The use, safety and cost of bariatric surgery before and after Medicare's national coverage decision. Ann Surg. 2011;254(6):860-5.

196. Myers VH, Adams CE, Barbera BL, Brantley PJ. Medical and psychosocial outcomes of laparoscopic Roux-en-Y gastric bypass: crosssectional findings at 4-year follow-up. Obes Surg. 2012;22(2):230-9.

197. Morton J, Brethauer S, Ponce J, Rosenthal R, Nguyen N. Bariatric Surgery Reduces National Healthcare Utilization In The Long-Term. Surg Obes Relat Dis. 2015;11(6):S3-4. 
198. Mor A, Omotosho P, Torquati A. Cardiovascular risk in obese diabetic patients is significantly reduced one year after gastric bypass compared to one year of diabetes support and education. Surg Endosc. 2014;28(10):2815-20.

199. Saez M, Barceló MA. Coste de la hipertensión arterial en España. Hipertens Riesgo Vasc. 2012;29(4):145-51.

200. Lopez-Bastida J, Boronat M, Moreno JO, Schurer W. Costs, outcomes and challenges for diabetes care in Spain. Glob Health. 2013;9:17.

201. Gould JC, Garren MJ, Starling JR. Laparoscopic gastric bypass results in decreased prescription medication costs within 6 months. J Gastrointest Surg. 2004;8(8):983-7.

202. Faria GR, Preto JR, Costa-Maia J. Gastric bypass is a cost-saving procedure: results from a comprehensive Markov model. Obes Surg. 2013;23(4):460-6.

203. Sampalis JS, Liberman M, Auger S, Christou NV. The impact of weight reduction surgery on health-care costs in morbidly obese patients. Obes Surg. 2004;14(7):939-47.

204. Zanela OO, Cabra HA, Meléndez G, Anaya P, Rupprecht F. Economic Evaluation of Bariatric Surgery in Mexico Using Discrete Event Simulation. Value Health Reg Issues. 2012;1(2):172-9.

205. Mason RJ, Moroney JR, Berne TV. The cost of obesity for nonbariatric inpatient operative procedures in the United States: national cost estimates obese versus nonobese patients. Ann Surg. 2013;258(4):541-551-553.

206. Liu B, Balkwill A, Spencer E, Beral V, Million Women Study Collaborators. Relationship between body mass index and length of hospital stay for gallbladder disease. J Public Health. 2008;30(2):161-6.

207. Lobo F. Políticas públicas para la promoción de la alimentación saludable y la prevención de la obesidad. Rev Esp Salud Pública. 2007;81(5):437-41.

208. Sánchez-Santos R, Estévez S, Tomé C, González S, Brox A, Nicolás R, et al. Training programs influence in the learning curve of laparoscopic gastric bypass for morbid obesity: a systematic review. Obes Surg. 2012;22(1):34-41. 
